University of South Florida

DIGITAL COMMONS

Digital Commons @ University of

@ UNIVERSITY OF SOUTH FLORIDA

South Florida

$11-16-2015$

\title{
Hepatic Nutrient and Hormonal Regulation of the PANcreatic- DERived Factor (PANDER) Promoter
}

Whitney Ratliff

University of South Florida, whitneyaratliff@gmail.com

Follow this and additional works at: https://digitalcommons.usf.edu/etd

Part of the Cell Biology Commons, Endocrinology Commons, and the Molecular Biology Commons

\section{Scholar Commons Citation}

Ratliff, Whitney, "Hepatic Nutrient and Hormonal Regulation of the PANcreatic-DERived Factor (PANDER) Promoter" (2015). USF Tampa Graduate Theses and Dissertations.

https://digitalcommons.usf.edu/etd/6020

This Dissertation is brought to you for free and open access by the USF Graduate Theses and Dissertations at Digital Commons @ University of South Florida. It has been accepted for inclusion in USF Tampa Graduate Theses and Dissertations by an authorized administrator of Digital Commons @ University of South Florida. For more information, please contact digitalcommons@usf.edu. 
Hepatic Nutrient and Hormonal Regulation of the PANcreatic-DERived Factor (PANDER) Promoter

by

Whitney A. Ratliff

A dissertation submitted in partial fulfillment

of the requirements for the degree of

Doctor of Philosophy

Department of Cell Biology, Microbiology and Molecular Biology College of Arts and Sciences

University of South Florida

\author{
Major Professor: Brant Burkhardt, Ph. D. \\ Patrick Bradshaw, Ph. D. \\ Meera Nanjundan, Ph. D. \\ Sandy Westerheide, Ph. D. \\ Date of Approval: \\ November 16, 2015
}

Keywords: FAM3B, ChREBP, Liver, Glucose, Transcription Copyright (C) 2015, Whitney A. Ratliff 


\section{DEDICATION}

This work is dedicated to my best friend, confidant, personal hero, doggy daddy and husband, Jeffery Ratliff. His words of encouragement and genuine interest in my research have provided me with the motivation to make this all possible. I would also like to dedicate this dissertation to my parents, Dennis and Kelley Campana, for their unwavering support throughout my academic career and their willingness to always be there for me when I need them, even from 1500 miles away. 


\section{ACKNOWLEDGEMENTS}

I would like to thank my mentor, Dr. Brant Burkhardt, for his support and advice in all aspects of life, scientific and otherwise. I would also like to express my genuine appreciation to the members of my committee: Dr. Patrick Bradshaw, Dr. Sandy Westerheide and Dr. Meera Nanjundan. Their guidance has been invaluable throughout this undertaking. Lastly, I would like to thank my labmates and friends: Melanie Kuehl, Mark Athanason, Katie Marelia, Tiffany Aguis, Alicia Chechele and Amanda Fernandez. Our mutual experience and struggle has brought us together and I sincerely appreciate all their love and support. I wish them all the best in their individual endeavors; they deserve all the success and happiness in the world. 


\section{TABLE OF CONTENTS}

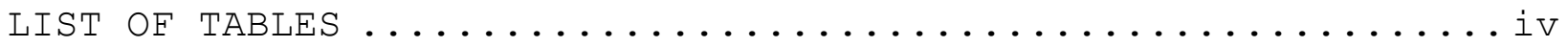

LIST OF FIGURES $\ldots \ldots \ldots \ldots \ldots \ldots \ldots \ldots \ldots \ldots \ldots \ldots \ldots \ldots \ldots \ldots$

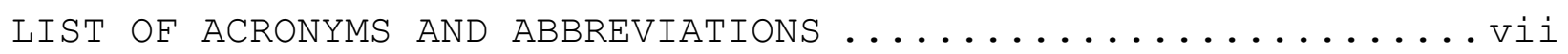

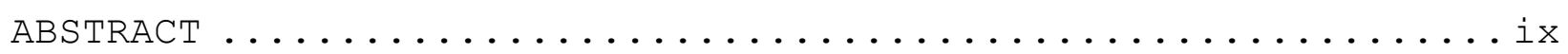

CHAPTER ONE: INTRODUCTION $\ldots \ldots \ldots \ldots \ldots \ldots \ldots \ldots \ldots \ldots \ldots \ldots$

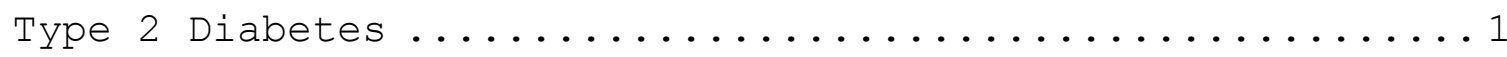

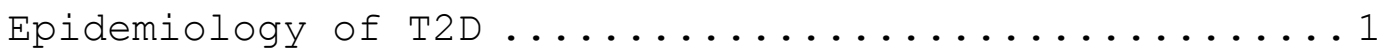

Clinical and molecular characteristics of T2D .......2

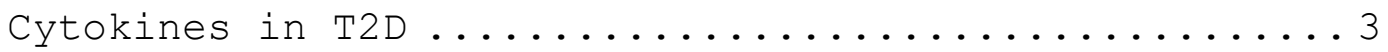

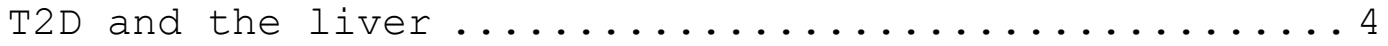

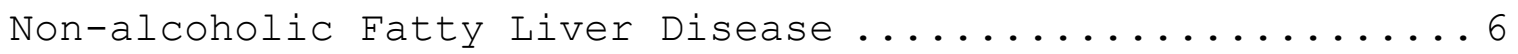

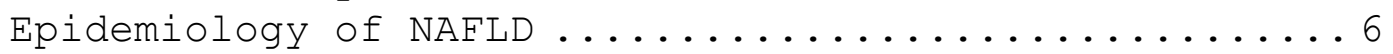

Clinical and molecular characteristics of NAFLD .....6

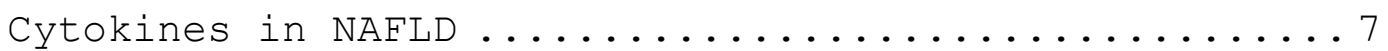

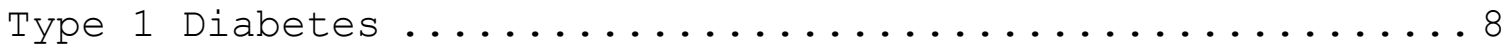

Initial Discovery of Pancreatic-Derived Factor .......... 10

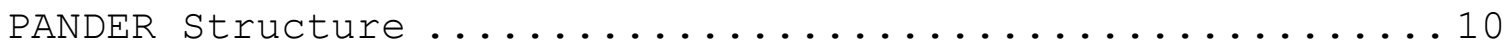

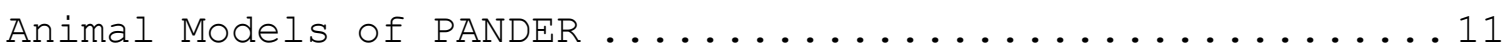

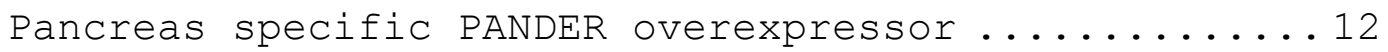

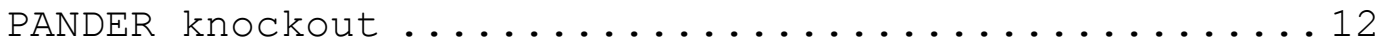

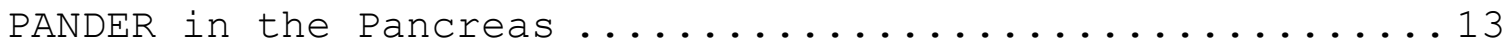

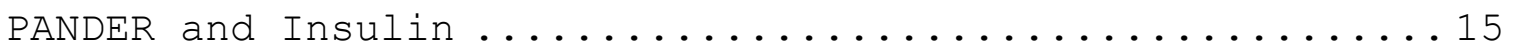

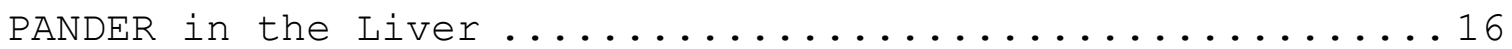

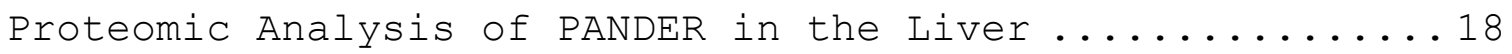

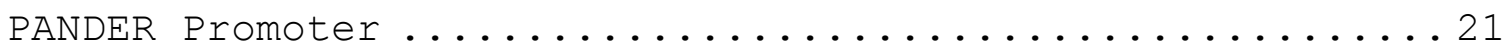

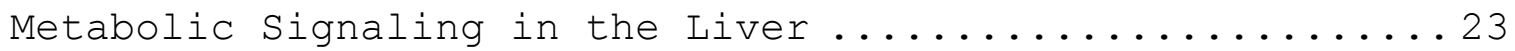

Carbohydrate Responsive Element Binding Protein .........24

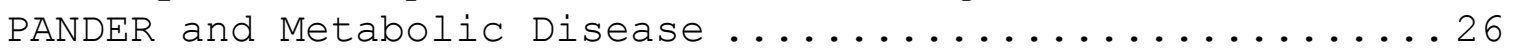

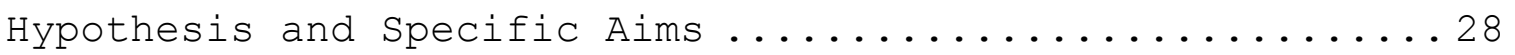

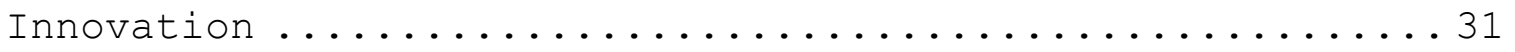

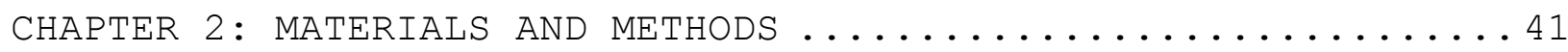
Identification of Transcriptional start Site ..........41 Identification of Putative Transcriptional Factor

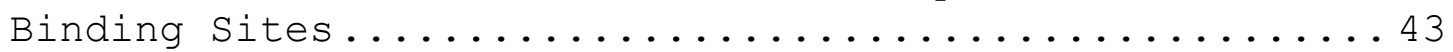

Western Analysis of PANDER Expression ................ 4 
Construction of the PANDER Promoter Luciferase

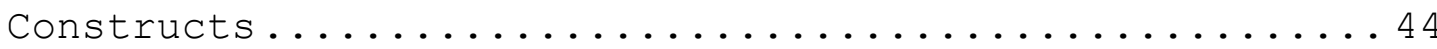

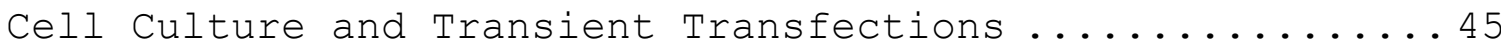

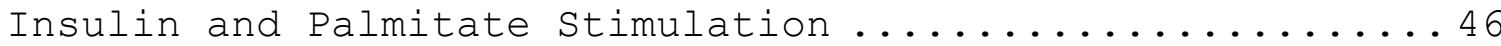

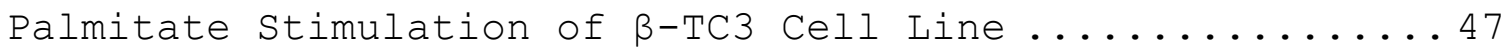

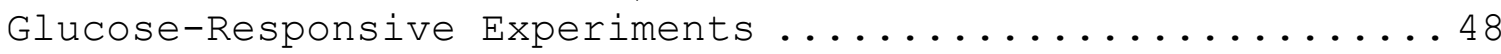

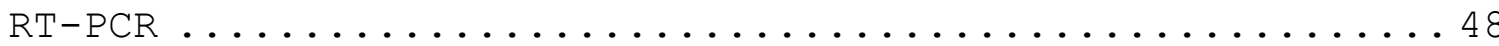

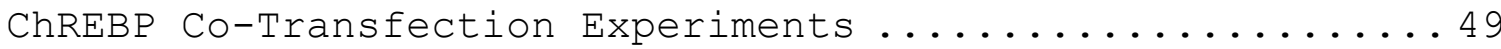

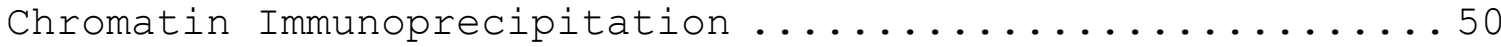

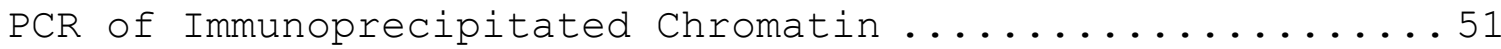

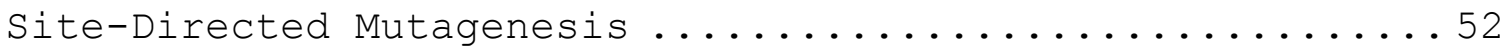

RT-PCR of PANDER Levels in Livers from Non-Obese

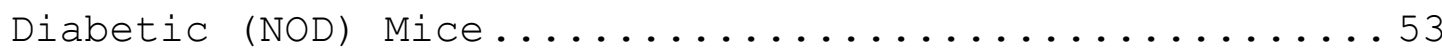

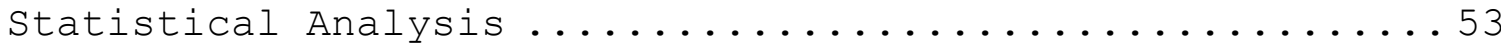

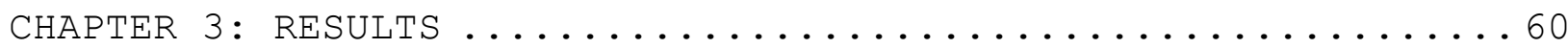

Identification of the Murine Hepatic PANDER

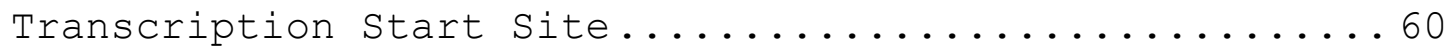

Cell-Specific Characterization of the PANDER Promoter ...61

Minimal Element of PANDER Promoter ...............63

Secretagogue-Mediated Expression of the Murine PANDER

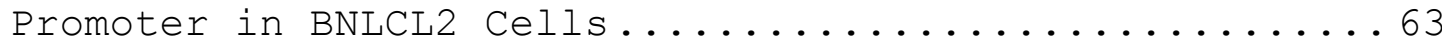

Palmitate Stimulation of $\beta$-TC3 Cell Line .............64

Minimal Element for Glucose-Responsiveness of the

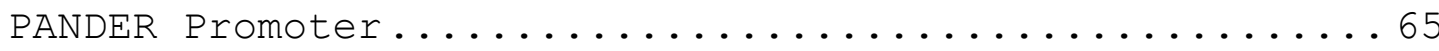

Glucose-Responsive PANDER mRNA Levels in Primary

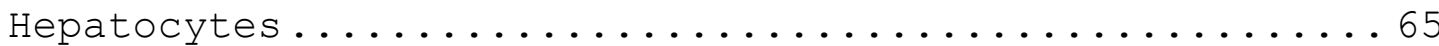

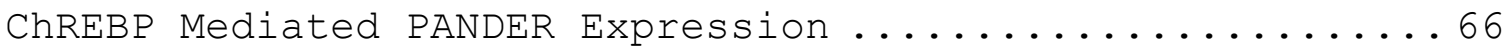

ChREBP Binding to the PANDER Promoter as Determined by

Chromatin Immunoprecipitation................67

E-box 3 Deletion Impairs Basal and Glucose-Stimulated

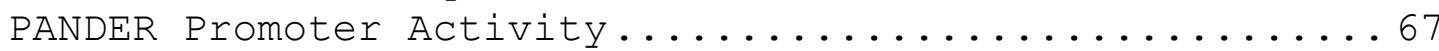

Hepatic PANDER Levels are Increased in Diabetic NOD

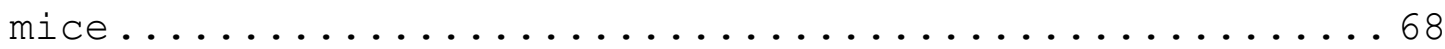

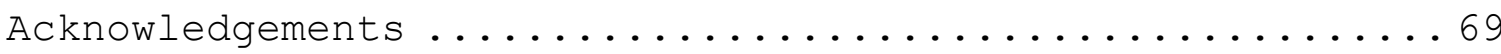

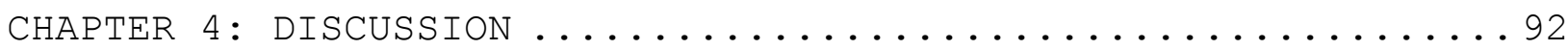

CHAPTER 5: PANDER INDUCED TRANSCRIPTION FACTOR PROFILING ....101

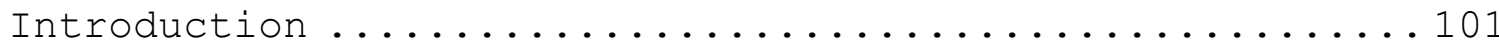

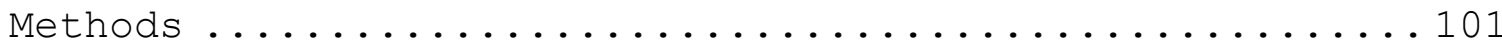

Primary hepatocyte culture and treatment ..........101

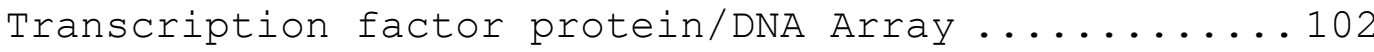

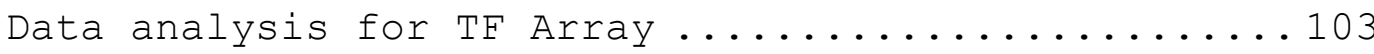

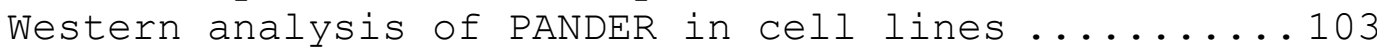

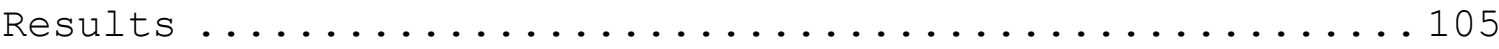


Identification of differentially expressed transcription factors in response to PANDER

treatment......................... 105

IPA analysis of differentially regulated proteins . 106

PANDER expression in cell lines ...............107

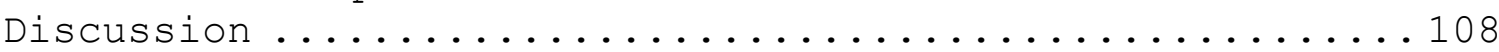

Significance of this study .................. 108

Canonical networks effected by PANDER treatment....109

Insulin resistance and cancer .................110

PANDER and insulin resistance .................. 112

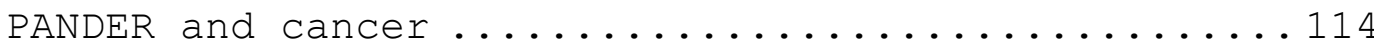

Acknowledgements .............................. 115

CHAPTER 6: IMPLICATIONS AND FUTURE DIRECTIONS ............ 131

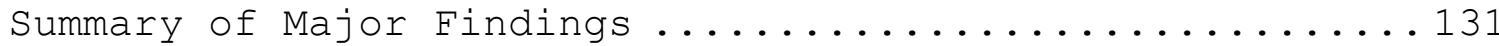

Chapter 3: Limitations of the Studies ................134

Chapter 5: Limitations of the Studies ................135

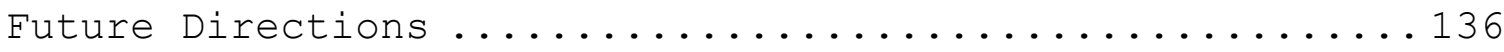

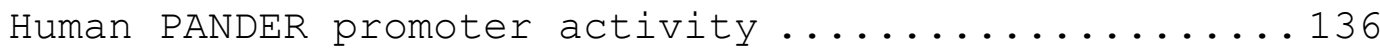

ChREBP regulation of PANDER in the liver ..........136

PANDER regulation in other cell types ............137

Other regulatory mechanisms of PANDER ............138

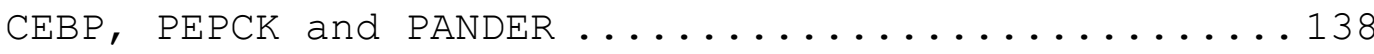

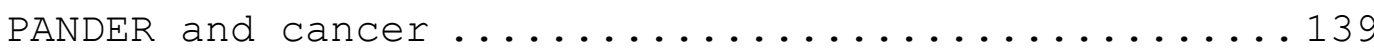

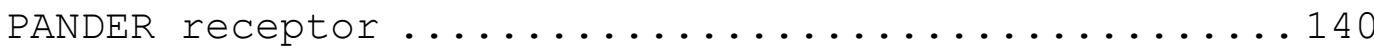

PANDER as a therapeutic target for T2D ........... 140

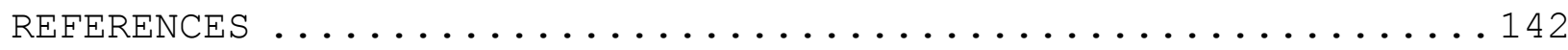

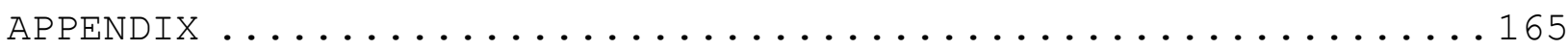




\section{LIST OF TABLES}

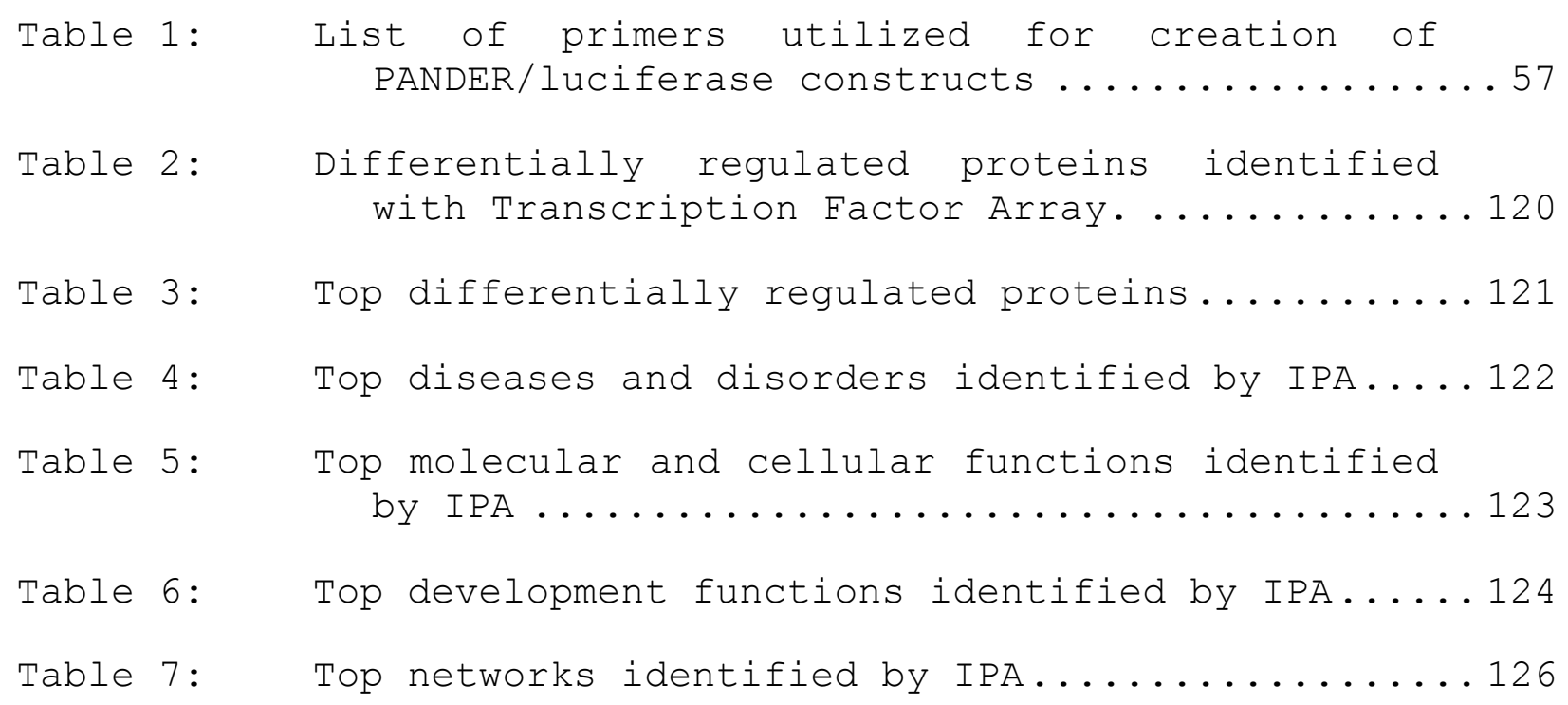




\section{LIST OF FIGURES}

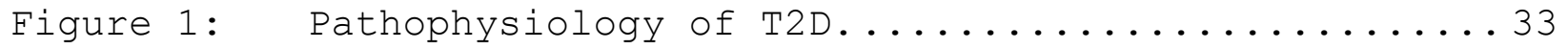

Figure 2: $\quad$ PANDER $\beta-\beta-\alpha$ Fold........................... 34

Figure 3: Increased PANDER levels during fed conditions.....35

Figure 4: Stable isotope labeling of amino acids in cell culture (SILAC) analysis of PANTG.............

Figure 5: Metabolic signaling in the liver in response to

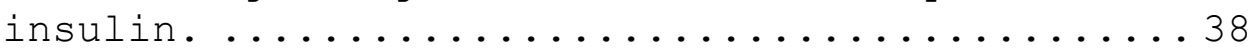

Figure 6: Regulation of ChREBP activity in the nucleus.....39

Figure 7: Schematic of Specific Aims...............40

Figure $8: \quad$ RLM-RACE procedure...................... 55

Figure 9: PANDER promoter luciferase constructs........56

Figure 10: Transfection Efficiency of BNLCL2, HepG2, C2C12

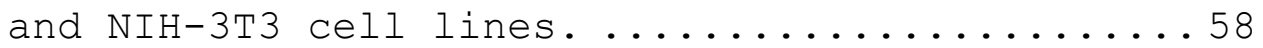

Figure 11: Acetyl-CoA Carboxylase promoter luciferase control plasmid activity, co-transfected with ChREBP and Mlx expression plasmids......59

Figure 12: Identification of the murine hepatic transcriptional start site.............70

Figure 13: Cell specificity and baseline activity of the murine PANDER promoter.................

Figure 14: PANDER promoter deletion analysis...........75

Figure 15: Insulin and palmitate inhibit PANDER promoter

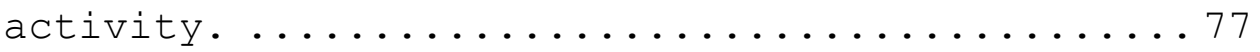

Figure 16: Palmitate Stimulation of $\beta$-TC3 cell line.......81

Figure 17: Glucose-responsiveness of the PANDER promoter....83 
Figure 18: ChREBP mediated activity of the PANDER

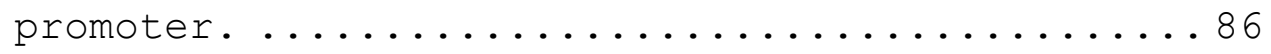

Figure 19: E-box 3 deletion decreases basal and glucose stimulated PANDER promoter activity........88

Figure 20: Hepatic PANDER levels in diabetic and non-

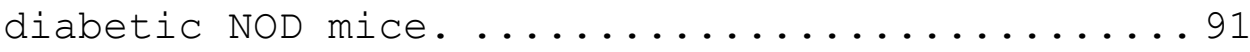

Figure 21: Overview of Transcription Factor Array........117

Figure 22: Diagram of Protein/DNA ComboArray from

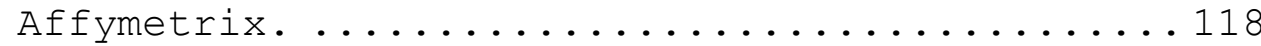

Figure 23: Transcription Factor Array membranes for untreated and pander treated hepatocytes.....119

Figure 24: Top liver toxicity functions identified by IPA...125

Figure 25: Gene expression, cellular development and embryonic development network...........127

Figure 26: PANDER protein levels in ovarian cancer cell

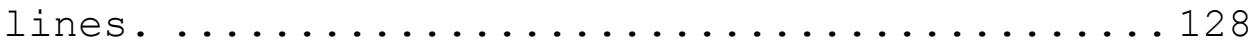

Figure 27: PANDER's activity within the insulin signaling

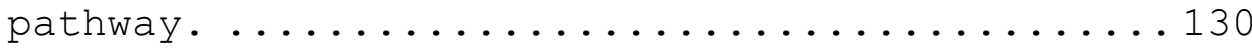




\section{LIST OF ACRONYMS AND ABBREVIATIONS}

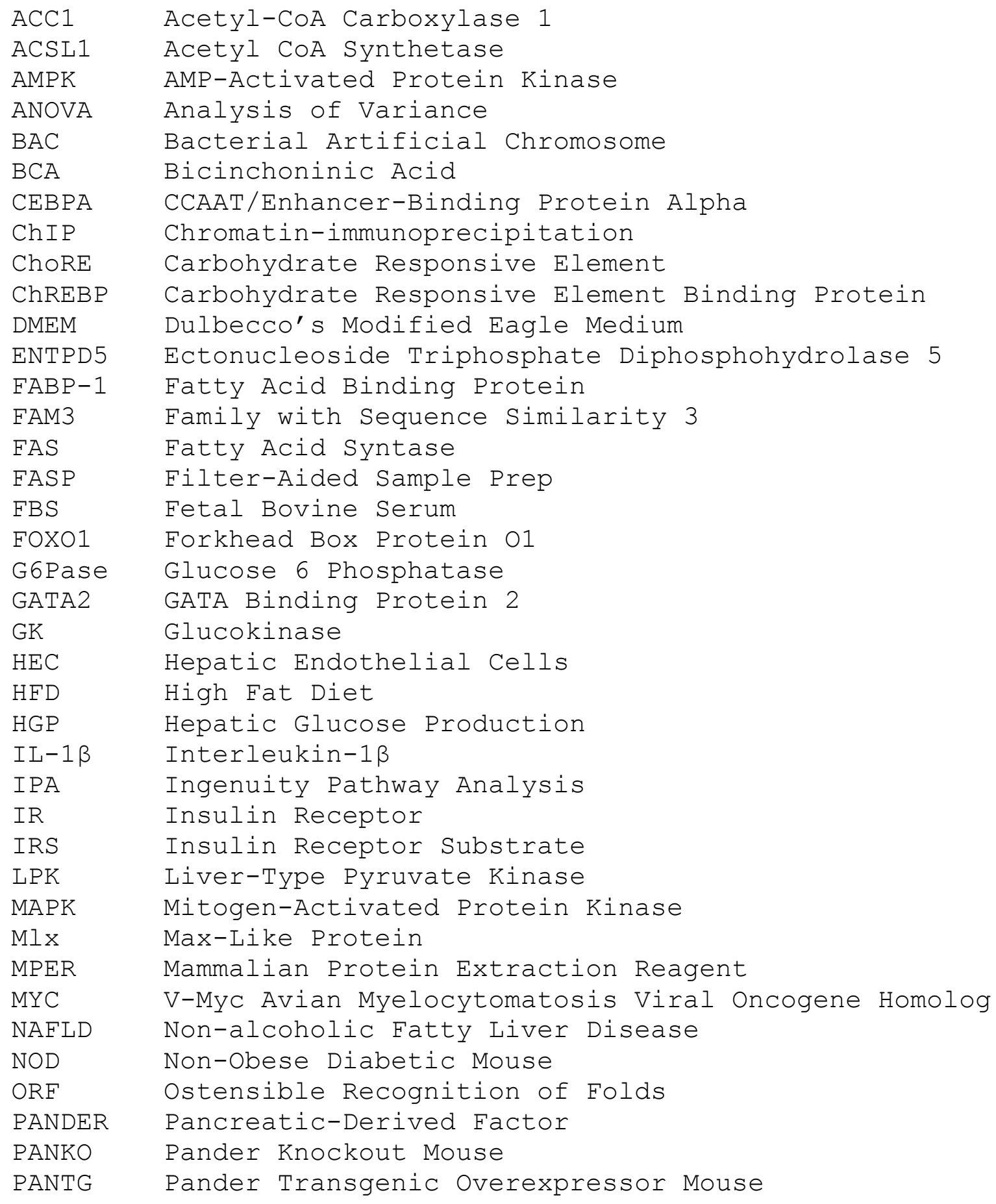




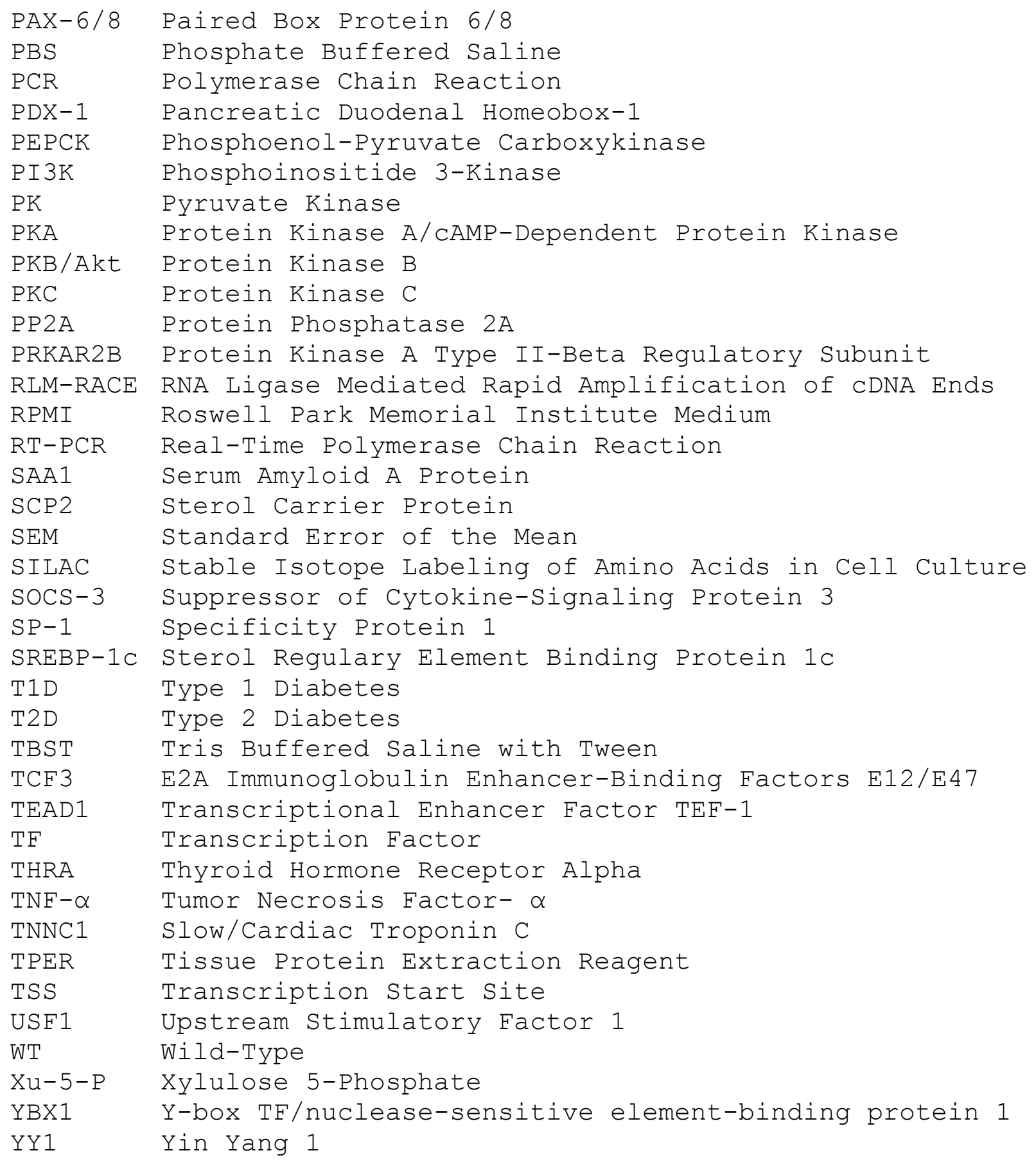




\section{ABSTRACT}

PANcreatic-DERived factor (PANDER, FAM3B) has been shown to regulate glycemic levels via interactions with both pancreatic islets and the liver. Although PANDER is predominantly expressed from the endocrine pancreas, recent work has provided sufficient evidence that the liver may also be an additional tissue source of PANDER production. At physiological levels, PANDER is capable of disrupting insulin signaling and promoting increased hepatic glucose production. As shown in some animal models, strong expression of PANDER, induced by viral delivery within the liver, induces hepatic steatosis. However, no studies to date have explicitly characterized the transcriptional regulation of PANDER from the liver. Therefore, our investigation elucidated the nutrient and hormonal regulation of

the hepatic PANDER promoter. Initial RNA-ligated rapid amplification of cDNA ends identified a novel transcription start site (TSS) approximately 26 bp upstream of the PANDER translational start codon not previously revealed in pancreatic $\beta$-cell lines. Western evaluation of various murine tissues demonstrated robust expression in the liver and brain. Promoter analysis identified strong tissue-specific activity of the 
PANDER promoter in both human and murine liver-derived cell lines. The minimal element responsible for maximal promoter activity within hepatic cell lines was located between -293 to 3 of the identified TSS. PANDER promoter activity was inhibited by both insulin and palmitate, whereas glucose strongly increased expression. The minimal element was responsible for maximal glucose-responsive and basal activity. Co-transfection reporter assays, chromatin-immunoprecipitation (ChIP) and sitedirected mutagenesis revealed that the carbohydrate-responsive element binding protein (ChREBP) increased PANDER promoter activity and interacted with the PANDER promoter. E-box 3 was shown to be critical for basal and glucose responsive expression. In summary, in-vitro and in-vivo glucose is a potent stimulator of the PANDER promoter within the liver and this response may be facilitated by ChREBP. 


\section{CHAPTER ONE : INTRODUCTION}

\section{Type 2 Diabetes}

Epidemiology of T2D. Worldwide, diabetes mellitus affects more than 285 million adults, approximately 6.4\% of the world population and estimates suggest that this number could soar to 439 million by the year 2030 [1]. In the United States alone, approximately 25.8 million people, $8.3 \%$ of the population, were affected by Diabetes in 2011 [2]. T2D, also known as "adultonset" diabetes, accounts for roughly 90-95\% of all diagnosed cases and has reached epidemic status in the US. The economic costs of diabetes are staggering, with costs in the United States at approximately \$174 billion dollars in 2007 alone [2]. More importantly, diabetes takes a massive toll on human lives with uncontrolled diabetes being associated with a substantially increased risk of premature morbidity and mortality [3, 4]. Diabetics are at an increased risk of cardiovascular disease, renal failure, blindness and amputation, leading to a decreased quality of life and, quite often, a premature death. The enormous societal and personal burden of the worsening diabetes epidemic underscores the importance of understanding the 
signaling proteins that contribute to T2D development and progression.

Clinical and molecular characteristics of T2D. T2D, also called "non-insulin dependent" diabetes, encompasses complex group of metabolic disorders that are characterized by increased blood glucose levels caused by insulin resistance and/or impaired insulin action [5, 6]. This can be due to impaired $\beta$-cell function or impaired sensitivity of target tissues to the action of secreted insulin [7, 8]. Under normal conditions, pancreatic $\beta$-cells produce insulin which is then stored in vacuoles until blood glucose levels become elevated. At this time, insulin is released into the blood stream and signals the uptake of glucose into skeletal muscle. Insulin signals for liver and skeletal muscle to convert glucose into its storage form, glycogen. Insulin action also results in the suppression of hepatic glucose production (HGP) and release of fatty acids from adipocytes $[6,9]$. In the diabetic state, impaired secretion of insulin from the $\beta$-cells causes decreased insulin signaling and results in a lack of glucose uptake causing hyperglycemia. Impaired ability of target tissues to respond to insulin results in impaired glucose tolerance (the ability for the body to clear glucose form the blood), increased hepatic glucose production and fatty acid release from adipose tissue. Meanwhile, the 
pancreas continues to produce insulin to combat the hyperglycemia, resulting in a hyperinsulinemic state. The resultant high levels of glucose and fatty acids in the blood causes a positive feedback loop furthering insulin resistance and resulting in worsened hyperglycemia, hyperinsulinemia and hyperlipidemia [8]. The pathophysiology of T2D is outlined in Figure 1. The lifestyle factors that contribute to the development and progression of T2D include high fat diet, inactivity and central adiposity, and have been studied extensively. New research into the molecular mechanisms of insulin resistance and $\beta$-cell dysfunction has underscored the importance of cytokines and other proteins in the development and treatement of T2D.

Cytokines in T2D. There has been a tremendous surge in research surrounding the importance of cytokines in the development and progression of diabetes. Abnormal cytokine profiles within the serum and pancreatic $\beta$-cells has been shown to play a role in the progression of both T1D and T2D [10]. Several cytokines have been implicated in the progression of T2D specifically,

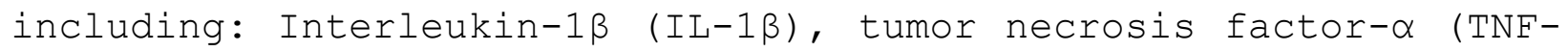
$\alpha)$, leptin and resistin. Chronic exposure of liver or muscle cells to these four cytokines has been shown to induce the expression of suppressor of cytokine-signaling protein 3 (SocS- 
3). socs-3 can bind to the insulin receptor (IR) and insulin receptor substrate (IRS)-1 and -2 , blocking the activation of insulin signaling [11, 12]. Furthermore, decreases in circulating adiponectin levels have been shown to be associated with the progression of $\beta$-cell dysfunction and insulin resistance in obesity [12]. The role of cytokines in the progression of T2D emphasizes the importance of further research into novel cytokines and factors that may be influencing cytokine induction and potentially increasing insulin resistance.

T2D and the liver. The liver is the main source of glucose output during the fasting state, controlling both glycogenolysis (the breakdown of glycogen into glucose for release into the bloodstream) [13] and gluconeogenesis (the generation of new glucose molecules from non-carbohydrate sources) [14]. As a result, the liver is one of the most significantly dysregulated organs in T2D due to the disruption of insulin signaling within the cells of the liver. This disruption leads to the failure to suppress HGP and further impairment of glucose tolerance [15, 16]. The failure of insulin to suppress HGP occurs through two separate mechanisms. Under normal conditions, insulin binds to its receptor directly on the liver cell membrane suppressing gluconeogenesis through inhibiting transcription of 
gluconeogenic enzymes glucose 6-phosphatase (G6P-ase) and PEPCK by nuclear exclusion of the Forkhead Box Protein o1 (FOXO1) transcription factor [17]. In addition to directly acting on the liver, insulin also inhibits the secretion of glucagon from pancreatic $\alpha$-cells though a mechanism that is yet to be fully elucidated. Recent research suggests this inhibition may be related to insulin-dependent activation of $\mathrm{GABA}_{\mathrm{A}}$ receptors via an Akt kinase-dependent pathway leading to hyperpolarization of the $\alpha$-cell and suppression of glucagon secretion [18]. Impaired insulin signaling has also been shown to result in the failure of adipocytes to suppress lipolysis (the breakdown of lipids though the hydrolysis of triglycerides into glycerol and free fatty acids). Insulin normally suppresses lipolysis within the adipocytes via both Akt-dependent and Akt-independent mechanisms [9, 19]. In the insulin resistant state, free fatty acids are released from the adipocytes and subsequently taken up by hepatocytes, thereby increasing both free fatty acid (FFA) content in the blood and within the liver itself (fatty liver) [20]. The increase in FFAs has also been shown to promote gluconeogenesis [21, 22] and contribute to further insulin resistance [23, 24], creating a dangerous positive feedback loop [25]. The dysregulation of these pathways in the diabetic state and their subsequent effect on overall liver metabolism has also led to research into T2D's association with other liver-related 
metabolic diseases and, in particular, Non-alcoholic Fatty Liver Disease, which often occurs concurrently with and as a precursor to T2D [26].

\section{Non-alcoholic Fatty Liver Disease}

Epidemiology of NAFLD. Chronic liver disease (CLD) is the $12^{\text {th }}$ leading cause of death in the United States [27]. Non-alcoholic fatty liver disease (NAFLD) is the most common form of CLD worldwide and is present in approximately 30\% of the population of the United States [28-30]. NAFLD is characterized by deposits of fat within the liver, or steatosis, which have been caused by factors outside of excessive alcohol use. This disease encompasses a range of liver conditions from minor hepatic steatosis to the high aggressive non-alcoholic steatohepatitis (NASH). NASH is known to cause hepatocyte injury, inflammation and fibrosis, eventually leading to the potential complications of liver cirrhosis and hepatocellular carcinoma [28]. Overall, NAFLD is associated with several serious comorbidities including: insulin resistance, obesity, hypertension and T2D $[31]$

Clinical and molecular characteristics of NAFLD. While the exact cause of NAFLD is unknown, the progression of the disease has been linked with multiple tissues and, in particular, insulin 
resistance within the liver and adipose tissues. The activated lipogenic signaling pathways associated with hepatic insulin resistance have been shown to be frequently associated with hepatic steatosis [32]. In addition, the insulin-resistant state causes an increase in lipolysis within the adipose tissues due to insulin's inability to suppress the activity of lipase. This leads to an increase in free fatty acids (FFA) within the blood serum, resulting in increased hepatic FFA uptake and the increased presence of triglyceride deposits within the liver [33]. Given the clear involvement of multiple tissues and the association with the increasing rates of $\mathrm{T} 2 \mathrm{D}$ and obesity throughout the world, the effort to determine and characterize the molecular mechanisms by which NAFLD develops and progresses has become integral to the overall understanding and treatment of the disease.

Cytokines in NAFLD. The role of cytokines in the development and progression of NAFLD and its subsequent comorbid conditions has been a major subject of research in recent years. Several cytokines have been implicated in the development of NAFLD and subsequent insulin resistant phenotype. Tumor Necrosis Factor $\alpha$ $(\mathrm{TNF}-\alpha)$ has been extensively researched and appears to play a central role in obesity-associated insulin resistance and fatty liver [34]. Levels of TNF- $\alpha$, circulating and within the liver 
and adipocytes, is increased in animal models of obesity [35, 36]. Treatment of cells in vitro with TNF- $\alpha$ has been shown to inhibit insulin action [37] and animals lacking in TNF- $\alpha$ or its receptors have been shown to have improved insulin sensitivity in obesity models [38]. Certain TNF- $\alpha$ polymorphisms have also been shown to be associated with levels of susceptibility for developing insulin resistance and NAFLD in humans [39]. In addition to TNF- $\alpha$, several other signaling molecules have been implicated in the development and progression of NAFLD, including: Jun N-terminal kinase (JNK) [40], IL-6 [41], adiponectin [42], and IL-8 [43]. Further study into these signaling molecules, and the characterization of novel factors, and their complex interaction leading to the development and progression of NAFLD and associated insulin resistance will be integral to the overall understanding of the disease and its comorbid conditions, as well as its prevention and treatment.

\section{Type 1 Diabetes}

Type 1 Diabetes (T1D), also formally referred to as "insulindependent" or "juvenile-onset" diabetes, accounts for 5-10\% of all cases of diabetes [44]. Approximately 78,000 children are diagnosed annually with T1D around the world [45]. Diagnosis in the United States is expected to increase by an estimated 144\% over the next 35 years from the current prevalence of 2.13/1000 
to 5.2/1000 by the year 2050 [46]. The estimated burden of T1D in the young in the U.S. ( $<20$ years) and Europe $(<15$ years) is expected to reach 400,000 by the year 2020 [46-48]. T1D is characterized by hyperglycemia caused by an autoimmune response resulting in the destruction of pancreatic $\beta$-cells [49]. Although onset of T1D can occur at any age, diagnosis is typically made during youth or young adulthood [50]. While the exact cause of this immune destruction remains elusive, recent research has made great strides in elucidating potential factors contributing to the development of this disease and its increased incidence in recent years. Possible explanations for the increase in diagnosis include: improved hygiene leading to reduced exposure to microbes and a resultant dysfunction in immune response $[51,52]$ and increased body size overloading $\beta-$ cells with insulin demand [53]. Much of the recent research into molecular factors associated with the development of T1D has revolved around the identification of genetic risk factors [54, 55] and biomarkers within the metabolome [56, 57]. Currently, the primary course of treatment for TlD is regular glucose monitoring and supplemental insulin and has been since the discovery of insulin in 1921 [58]. While supplemental insulin has proved a successful treatment for many patients, it is not without its drawbacks, including acute morbidity and mortality as well as serious complications [59, 60]. Thus, further 
research into the molecular factors, which could provide insight into early diagnosis and more effective treatment, will be of the utmost importance over the next several years as TID prevalence continues to rise.

\section{Initial Discovery of Pancreatic-Derived Factor}

Pancreatic-Derived Factor (PANDER) is a recently characterized secreted hormone. The discovery of PANDER was made through the use of the algorithm Ostensible Recognition of Folds (ORF) in a search for novel cytokines based on predicted secondary structure [61]. The predicted secondary structure of PANDER revealed the four helix bundle with up-up-down-down topology that is typical of many other common cytokines such as IL-2, -10 and -13 [62]. This algorithm identified a novel family of proteins predicted to display this cytokine-like secondary structure: family with sequence similarity 3 (FAM3). The FAM3 family consists of four members: FAM3A, FAM3B, FAM3C, FAM3D [63].

\section{PANDER Structure}

Although initial data suggested that FAM3 family members had a cytokine like structure, recent data suggests that the FAM3 family represents a novel class of signaling molecules, with PANDER exhibiting a globular $\beta-\beta-\alpha$ fold (Figure 2). This unique 
structure is conserved among the FAM3 proteins and is unlike that of any known cytokine [64]. The amino acid sequence homology among the four FAM3 members is approximately $31.6-$ 53.3\%, with there being no significant homology to any other known cytokines [65]. FAM3B was later coined as PANDER due to its primary expression in the endocrine pancreas. PANDER has also been shown to be expressed in lower levels in the prostate, intestine, and ovaries [65-67]; although the function of PANDER in these other tissues is unknown. Human PANDER contains 235 amino acids and shares 78\% primary sequence homology, including 4 conserved cysteines, with its murine homolog [68]. Given PANDER's structure, it is reasonable to surmise that PANDER plays an important role in cellular signaling pathways within the pancreas and in the liver.

\section{Animal Models of PANDER}

Several animal models have been derived to investigate PANDER's potential role in glucose regulation, with differing results. One group evaluated hepatic PANDER overexpression achieved through adenoviral delivery. This model revealed fasting hyperglycemia, hyperinsulinemia and elevated corticosterone levels [69]. Another group derived a similar adenoviral delivered overexpressing model with different results and did not observe glucose intolerance, fasting glycemia, or elevated 
corticosterone levels. However, liver and serum triglyceride content was increased compared to WT mice along with serum insulin levels [67].

Pancreas specific PANDER overexpressor. To help elucidate the sources of these discrepancies and provide more conclusive evidence of the effect of PANDER overexpression, our laboratory has created and phenotyped the only transgenic mouse model (PANTG) with tissue-specific PANDER overexpression and secretion from the endocrine pancreas to further elucidate the physiologic role of pancreas-secreted PANDER in-vivo. Male PANTG mice display elevated fasting glucose and insulin. Hyperinsulinemiceuglycemic clamp studies also revealed that PANTG displays increased hepatic glucose production and insulin resistance compared to WT counterparts [70]. Our metabolic phenotyping and proteomic analysis strongly indicate that endocrine specific overexpression of PANDER impacts hepatic glycemic output and lipid regulation [67].

PANDER knockout. In addition to the creation of a PANDER overexpressor, we also have access to a global PANDER knockout mouse (PANKO). In this mouse, the PANDER gene has been disrupted by replacing the region containing the first two exons, transcriptional start site and the secretion signal peptide, 
with the neomycin gene. This results in the complete loss of PANDER expression in all tissues. The PANKO mouse displays glucose intolerance during a glucose tolerance test due to impaired insulin secretion and abnormal $\mathrm{Ca}^{2+}$ handling within pancreatic islets [67]. In addition, during hyperinsulinemiceuglycemic clamp studies, PANKO displayed lower hepatic glucose production (HGP) and increased HGP suppression when compared to wild-type [67], as well as improved hepatic insulin sensitivity [71]. This phenotype was more significant in male PANKO mice, however, females also demonstrated the overall trends in enhanced glucose tolerance [71]. These animal models provide invaluable evidence of the in vivo effects of overexpressing and knocking out PANDER expression, and may prove useful in the study of the effects of PANDER on the liver.

\section{PANDER in the Pancreas}

Initial characterization of PANDER's tissue distribution by Northern Blot revealed that both human and murine PANDER are highly expressed in the pancreas [65]. Immunohistochemical staining has shown PANDER to be localized specifically to the islet of Langerhans in the endocrine pancreas, with no expression in the exocrine pancreas [68]. PANDER has been shown to act on the cells of the pancreas in a number of ways. In vitro, overexpression of PANDER or treatment with recombinant 
PANDER led to apoptosis in rat, mouse and human pancreatic $\beta-$ cells [68, 72]. Microarray analysis revealed a significant downregulation of cyclin-dependent kinase inhibitor 1A, also known as p21. The downregulation of this protein has been shown in other cell types to mediate cell death [73]. This analysis also revealed activation of caspase-3, which has been shown previously to be a central molecular involved in $\beta$-cell death in autoimmune diabetes [74]. Caspase-3 has also been demonstrated to cleave p21, which is important for death-associated cyclin A/Cdk2 activation in several cell types [75]. Taken together, the decrease in p21 and increase in caspase-3 may be playing a role in PANDER-induced cell death in islets [76]. Recent research suggests that PANDER has an effect on insulin secretion from the endocrine pancreas, leading to changes in glucose metabolism. PANDER-deficient mice display glucose intolerance and decreased serum insulin levels in response to glucose or arginine administration [77]. PANDER ${ }^{-/-}$islet cells have decreased insulin secretion in response to glucose or calcium chloride stimulation. Previous data suggest that decreased glucose-stimulated insulin secretion in rat models of $\mathrm{T} 2 \mathrm{D}$ can be attributed to abnormal calcium handling within the islets [78, 79]. PANDER ${ }^{-/-}$islets also display an abnormal intracellular calcium response during glucose stimulation when compared to PANDER $^{+/+}$islets [77]. These islets do not display the typical 
calcium dip during glucose stimulation. This dip has been attributed to SERCA sequestration of $\mathrm{Ca}^{2+}$ in $d b / d b$ murine islets [80]. Though SERCA mRNA levels were similar between PANDER $^{-/}$and wild-type mice, activity was not evaluated and may provide more insight into the mechanism behind the abnormal calcium response in the absence of PANDER [77]. Overall, these data suggest a role of PANDER in regulating insulin secretion in pancreatic islets by affecting calcium homeostasis under physiological conditions.

\section{PANDER and Insulin}

PANDER is localized to the insulin secretory vesicles in $\beta-$ cells, suggesting that it may be cosecreted with insulin [81]. Pancreatic $\beta$-cell lines treated with glucose saw marked increases in both PANDER and insulin in a dose and time dependent manner [82]. In fact, several secretagogues and inhibitors of insulin secretion were evaluated and it was found that stimulators of insulin secretion also induced PANDER secretion, while inhibitors repressed PANDER secretion in mouse islets and $\beta$-cell lines. Correct structure and conformation of PANDER is essential for PANDER secretion from $\beta$-cells. Misfolded PANDER mutants can be produced by and accumulate within the cell but are unable to be sorted into insulin secretory granules and secreted from the cell [82]. It is unknown how these mutants may 
be cleared from the cell and/or targeted for degradation. These data suggest that the expression and secretion of PANDER and insulin within pancreatic $\beta$-cells are strongly coupled. PANDER has also been shown to localize within $\alpha$-cells, to a glucagonnegative granular cytosolic compartment, cytoplasmic granules, neuroendocrine vesicles, endoplasmic reticulum (ER), and the cis-Golgi [83]. The secretion of PANDER from $\alpha$-cells can be induced by insulin and requires PI3K/Akt signaling via stimulation of the insulin receptor [83]. PANDER's localization and action on both $\alpha$ - and $\beta$-cells suggest a strong functional role of PANDER within the endocrine pancreas; however, given its association with insulin and glucose, it stands to reason that PANDER may also play a role in other metabolic tissues and, in particular, the liver.

\section{PANDER in the Liver}

In vitro saturation binding assays have demonstrated ${ }^{125}$ I-PANDER binding to the liver cell membrane [84]. These results suggest that the liver is a novel target tissue of PANDER, although PANDER receptor has yet to be identified. In vitro research using a human liver-derived cell line (HepG2) indicates PANDER suppresses hepatic insulin signaling by decreasing levels of tyrosine phosphorylation of insulin receptor (IR) and insulin receptor substrate (IRS-1) and repressing the insulin-stimulated 
activation of PI3K and Akt [84]. Furthermore, it has been shown that recombinant murine PANDER treatment induces gluconeogenic gene expression and increased glucose production in primary cultured mouse hepatocytes [69]. In vivo, mice overexpressing PANDER in the pancreatic islets (PANTG) are glucose intolerant due to impaired insulin signaling within the liver [69, 77]. Recent data from our lab also indicates that hepatic PANDER protein is upregulated in the fed state as well as being chronically elevated in the PANTG mouse (Figure 3) [70]. These data suggest that islet-derived and recombinant PANDER are able to target the tissues within the liver and induce hepatic glucose production while inhibiting hepatic insulin signaling However, the role that liver-derived PANDER has on hepatic insulin signaling has not been well characterized. One study showed significant activity of the PANDER promoter within liverderived cell lines, even more significant than that of isletderived cell lines, although this finding was not explored further [66]. This study brings to light the potential importance of the liver in PANDER secretion in addition to it being a target for PANDER action. A recent study showed that PANDER expression in the liver may play a role in promoting lipogenesis and downregulating insulin signaling in response to insulin stimulation [67]. While recent data has supported liverderived PANDER as being an important factor in regulating 
insulin signaling and hepatic glucose production, the mechanism of regulation and functional significance of liver-derived PANDER remains largely unexplored.

\section{Proteomic Analysis of PANDER in the Liver}

One means of evaluating the function of PANDER within the liver is be examining changes in global protein expression in liver tissue when exposed to varying levels of PANDER. Our lab has performed initial proteomic analysis of the pancreas-specific PANDER overexpressor mouse (PANTG) [70]. Initial characterization of the PANTG mouse revealed increased fasting and post-prandial glycemic levels in the absence of peripheral insulin resistance but the presence of hepatic insulin resistance. We then measured critical hepatic insulin signaling molecules via a comprehensive proteomic approach known as stable isotope labeling of amino acids in cell culture (SILAC). This quantitative proteomic strategy metabolically labels the entire proteome and allows for identification and network elucidation by mass spectrometry analysis and has become a highly useful and relatively novel tool to identify complex protein mixtures, signaling cascades, and regulated protein modifications [85]. Total protein isolate was prepared from extracted livers from PANTG and WT mice ( $\mathrm{n}=6$ per group) obtained during fed conditions. Isolated liver lysate was prepared from PANTG and WT 
male mice ( $\mathrm{n}=6$ per group) during random fed conditions. Livers were homogenized in TPER buffer (Thermo Fisher Scientific) followed by high-speed centrifugation. Ethanol-treated AML-12 cells were utilized as a surrogate cell line for protein comparison as previously described [85]. The AML-12 cells were heavy labeled by culturing in media containing ${ }_{13} \mathrm{C}_{6}$ L-Lysine-2-HCl and ${ }_{13} \mathrm{C}_{6}$ L-arginine-HCl followed by homogenization in MPER buffer (Pierce, Rockford, IL) followed by high-speed centrifugation. Supernatants were collected and protein concentration quantified (Pierce BCA protein assay). Equivalent amount of liver protein was then spiked with equal mass of protein from AML-12's. The spiked mixture was solubilized using FASP protein digestion kit (Protein Discovery). The six samples derived from PANTG and WT livers, respectively, were then batched and both samples were desalted prior to fractionation using an automated CXS column. Nine fractions were selected for both PANTG and WT mice and subsequently dissolved in 1\% aqueous formic acid for evaluation by mass spectrometry (LTQ Orbitrap). Data was normalized and analyzed using the Ingenuity Pathway Analysis (IPA) program. A total of 1640 proteins were confidently identified and quantitated. Relative changes in the levels of identified proteins were determined by the ratio of PANTG to that of WT and normalized to Tubulin B5. Of those, 88 protein groups were upregulated and 9 were downregulated $(P<0.05$ for both groups). 
The distribution of ratios of all quantitated proteins indicated excellent representation of the proteome between PANTG and WT ratio relative to that of AML-12 cells. Approximately 1,640 proteins were identified and quantitated. Data was normalized and analyzed using the Ingenuity Pathway Analysis (IPA) program. Relative changes in the levels of identified proteins were determined by the ratio of PANTG to that of WT and normalized to proteomic expression of Tubulin B5. Of those, 88 protein groups were up-regulated and 9 were down-regulated $(P<0.05$ for both groups). IPA of the differentially expressed proteins identified lipid metabolism as one of the top associated network functions as significantly up-regulated (20 total proteins with $P=9.46 \times 10^{-6}$ ) with an overall score of 57 (Score of 2 is significant) (Figure 4A). Further IPA analysis identified quantity of triacylglycerol to be in a predicted increased state (z-score of 2.4) with a genetic network of interacting proteins consisting of consisting of: fatty acid binding protein (FABP1), protein kinase A type II-beta regulatory subunit (PRKAR2B), phosphoenol-pyruvate carboxykinase (PEPCK), sterol carrier protein (SCP2), acetyl CoA synthetase (ACSL1), and ectonucleoside triphosphate diphosphohydrolase 5 (ENTPD5) (Figures 4B and 4C). Of those identified to promote increased triglyceride production, 4 of which were revealed to molecularly interact as determined by IPA (Figure 4C). Overall, SILAC 
identified a proteomic profile within the PANTG liver of increased lipid metabolism and increased PEPCK (gluconeogenic) expression supporting the observed results obtained with both the HEC and phenotypic analysis [70]. The SILAC approach used in these preliminary studies may be useful in further evaluating the function of PANDER within the liver.

\section{PANDER Promoter}

PANDER promoter activity has been studied previously, including the identification of a transcriptional start site in the $\beta$-TC3 mouse insulinoma cell line. The transcriptional start site was identified 520 bp upstream of the translational start codon by 5'-RLM-RACE [66]. RLM-RACE is a PCR-based technique that is designed to amplify cDNA from full-length, capped mRNA. The resultant cDNA can then be sequenced to identify a transcriptional start site [86]. Further analysis of the promoter sequence revealed three A-box elements and three E-box elements within the $-338 /+491$ promoter region (relative to the transcriptional start site). In order to assess promoter activity within specific tissues and under certain conditions, several promoter constructs were created. These constructs contain truncated regions of the PANDER promoter conjugated to the luciferase gene within the pGL3-basic plasmid. This allowed the evaluation of PANDER promoter activity using a standard 
luciferase assay from transfected cell lysates. The PANDER promoter constructs that were originally created included the following regions: $\quad-2338 /+491,-1338 /+491, \quad-338 /+491$ and +1/491. The PANDER promoter region was flanked with KpnI and MIuI restriction sites via PCR to aid in the insertion of the promoter construct into the pGL3-basic luciferase construct [66]. Later, several more promoter construct variations were created for further evaluation of promoter activity, including: $-338 /+1,+1 /+100,+1 /+200,+100 /+491,+200 /+491$. Using these promoter constructs, the PANDER promoter was found to be highly active in islet-derived cell lines as well as a murine liverderived cell line (BNLCL2), although the significance of promoter activity in the liver was not further explored [66]. This study did find, however, that the PANDER promoter was glucose-responsive in islet-derived cell lines. Further study revealed that the glucose responsiveness of the PANDER promoter in islets may be regulated primarily through transcription factor binding to A-box elements on the promoter. Pancreatic Duodenal Homeobox-1 (PDX-1) was found to produce the most significant increase PANDER promoter activity among the transcription factors investigated, through its binding to A-box elements in response to glucose stimulation [87]. While these data provide some insight into regulation of the PANDER promoter in the pancreas, there have been no studies investigating the 
regulation of the PANDER promoter in the liver, despite PANDER's potentially important role in regulating hepatic insulin signaling and glucose production. A previous study has identified three E-box elements with the +200/+491 region of the PANDER promoter, which may serve as binding sites for glucoseinduced transcription factors [82]. The PANDER promoter contains several potential nutrient and hormonal binding sites that have yet to be characterized and our studies will be the first to reveal the potential mechanism of PANDER expression within the liver.

\section{Metabolic Signaling in the Liver}

In the fed state, blood glucose concentration is increased which results in the rapid release of insulin from pancreatic $\beta$-cells. This induces the anabolic pathways in insulin's target tissues and, in particular, the liver. The insulin signaling cascade is initiated when insulin binds to the extracellular $\beta$-subunits of the dimerized insulin receptor on the liver cell membrane [88]. This binding induces the autophosphorylation of tyrosine residues at the intracellular $\beta$-subunit of the insulin receptor. IRS proteins bind to the phosphorylated receptor via their phosphotyrosine binding (PTB) domain and are subsequently phosphorylated on multiple residues creating docking sites for src homology 2 domain containing proteins such as insulin 
receptor-1, insulin receptor-2, and phosphoinositide-3 (PI-3) kinase. This signal is then transferred onto further molecules such as Akt and protein kinase C (PKC) to potentiate various insulin-stimulated anabolic actions. Akt action inhibits glycogen phosphorylase, thereby inhibiting glycogenolysis [89]. Akt also phosphorylates FOXO1, which creates binding sites for 14-3-3, preventing DNA binding and transcription of gluconeogenenic proteins, such as PEPCK and G6Pase [90]. PKC activates sterol regulatory element binding protein 1c (SREBP1c) which, in turn, activates transcription of lipogenic (FAS) and glycolytic proteins (glycokinase (GK) [91], pyruvate kinase (PK) [92]) and inhibits gluconeogenic factors (PEPCK, G6Pase [93]). It has also been shown that another protein, carbohydrate responsive element binding protein (ChREBP) works alongside SREBP-1C to synergistically activate these pathways [94]. Overall, the hepatic action of insulin results in three primary functions regulation of lipid metabolism, activation of glucose storage and shutdown glucose production and output, and inhibition of gluconeogenesis. The mechanism of these actions is outlined in Figure 5.

\section{Carbohydrate Responsive Element Binding Protein}

Several E-box elements have been identified within the $+200 /+491$ region of the PANDER promoter. These E-box elements may comprise 
a carbohydrate responsive element (ChoRE) where binding of carbohydrate responsive element binding protein (ChREBP) could occur [95]. ChREBP is a transcription factor that has been shown to induce transcription of several genes associated with carbohydrate metabolism in response to glucose stimulation within the liver [96-98]. Under non-stimulatory conditions, ChREBP is phosphorylated and sequestered within the cytosol by association with the regulatory protein 14-3-3. Influx of glucose with the hepatocyte leads to an increase in xylulose 5phosphate (Xu-5-P) levels, subsequently activating protein phosphatase 2A (PP2A). PP2A dephosphorylates ChREBP, allowing it to be translocated to the nucleus where it dimerizes with maxlike protein $\mathrm{X}(\mathrm{Mlx})$ and binds to the ChoRE of target genes such as FAS, L-PK and ACC [98, 99]. Increased levels of glucagon and fatty acids lead to increased activity of PKA and AMPK, respectively, which phosphorylate ChREBP and promote its exclusion from the nucleus (Figure 6) [98, 100, 101]. Studies have revealed that glucose may be responsible for inducing lipogenesis in the liver through this stimulation of ChREBP [102, 103]. These studies showed, using primary rat hepatocytes, that insulin and high glucose levels resulted in a significant increase in acetyl-coA carboxylase 1(ACC1), fatty acid synthase (EAS) and S14, all of which are targets of ChREBP and are involved in the induction of lipogenesis in the liver [103]. In 
addition to lipogenesis, ChREBP appears to be involved in the induction of liver glycolysis. ChREBP has been found to bind and induce the transcription of the liver-type pyruvate kinase (LPK) gene, which is essential in the induction of glycolysis in the liver [104]. In vivo research showed that mice deficient in ChREBP had decreased levels of LPK and reduced liver glycolysis, in addition to a marked reduction in lipogenesis [102]. Still another study found that ob/ob mice treated with recombinant adenovirus containing shRNA against ChREBP displayed improvements in both hepatic steatosis and insulin sensitivity [105]. Given the potential ChoRE within the PANDER promoter and its important role in glucose-induced lipogensis in the liver, ChREBP may serve as an important stimulator of PANDER expression in the liver in response to increased levels of glucose.

\section{PANDER and Metabolic Disease}

PANDER's potential role in metabolic disease is multifaceted. It has been shown to be involved in all three major hallmarks of T2D: hyperglycemia, hyperinsulinemia and hyperlipidemia. Research suggests that PANDER may be co-secreted with insulin in the hyperglycemic state [81, 82] wherein it binds to the liver cell membrane and contributes to hepatic insulin resistance, lipogenesis [84] and gluconeogenesis [69]. Hepatic

overexpression of PANDER has resulted in various pleiotropic 
effects producing a selective hepatic insulin resistant phenotype of impaired hepatic insulin signaling yet increased hepatic triglycerides and production of very low-density lipoprotein (VLDL)-TG [69]. Similar results were observed in a PANDER transgenic pancreatic $\beta$-cell specific overexpressing model that exhibited decreased glucose tolerance, hepatic insulin resistance yet increased hepatic TG concentration [70]. Taken together, the display of these various animal model phenotypes that have been further supported by PANDER knockout studies $[70,71,77$, 106] has potentially implicated PANDER's involvement in the initiation and/or progression of T2D [107] and NAFLD [108]. Additionally, a very recent study demonstrated that PANDER levels within patients with metabolic syndrome were increased and highly correlated with severity of the metabolic syndrome [109]. These factors, combined with evidence showing that PANDER may induce apoptosis in pancreatic $\beta$-cells [68, 76], may also implicate PANDER as a potential biomarker in TID. Despite this critical importance, the hepatic regulation of PANDER has not been examined particularly in the context of which nutrient or hormonal secretagogues or transcription factors govern expression. Therefore, the work contained within this dissertation is the first to characterize the transcriptional regulation of hepatic PANDER. 


\section{Hypothesis and Specific Aims}

In 2011, approximately 25.8 million people, 8.3\% of the population, were affected by Diabetes mellitus in the United States [2]. T2D accounts for roughly 90-95\% of all diagnosed cases and has reached epidemic status in the US. The economic and human costs of T2D are staggering with uncontrolled diabetes being associated with a substantially increased risk of premature morbidity and mortality [3, 4]. The worsening diabetes epidemic underscores the importance of understanding the mechanisms by which diabetes develops and progresses. Pancreatic-Derived Factor (PANDER) is a novel cytokine-like hormone that interacts with the endocrine pancreas and the liver and is associated with the regulation of insulin secretion and hepatic glucose production [82]. Islet-derived and recombinant PANDER has been shown to decrease hepatic insulin signaling and increase hepatic glucose production, both in vitro and in vivo [77, 84]. PANDER's role in regulating insulin signaling and glucose production suggests that it may play an important role in the development and progression of T2D. While PANDER has been studied almost entirely within the context of secretion from pancreatic islets, a recent and novel study indicates that liver-derived PANDER may also play an important role in regulating insulin signaling. Liver-derived PANDER has been shown to induce lipogenesis and insulin resistance in response 
to insulin stimulation and play a potential role in the deleterious cascade of selective hepatic insulin resistance [67]. This novel finding emphasizes the importance of further evaluating PANDER's role within the liver. The proposed project will seek to elucidate the mechanism by which PANDER is transcriptionally regulated in the liver. We hypothesize that PANDER expression within the liver can be induced by both nutrient and hormonal secretagogues and is tightly regulated by transcription factor binding to the PANDER promoter. A better understanding of how PANDER expression is regulated in the liver will lead to further characterization of mechanisms by which insulin signaling and glucose production become dysregulated in human disease, in particular T2D. Specifc Aims are depicted in Figure 7 .

Specific Aim 1: Determine secretagogues responsible for PANDER transcriptional expression

1.1 Measure the role of insulin, glucose and palmitate on PANDER promoter activity via luciferase assay

1.2 Identify the hepatic transcriptional start site within the PANDER promoter using RNA Ligase Mediated Rapid Amplification of CDNA Ends (RLM-RACE)

1.3 Determine the intracellular distribution of PANDER within the liver 
Specific Aim 2: Identify transcriptional factors mediating PANDER expression in the liver

2.1 Perform computational search to identify putative transcriptional factors

2.2 Identify critical transcriptional factors by cotransfection experiments

2.3 Determine functionality of critical transcriptional factor binding regions

Specific Aim 3: Examine PANDER induced transcription factor profile and canonical pathways within primary hepatocytes.

3.1 Perform PANDER treatment and transcription factor array on primary hepatocytes

3.2 Identify differentially regulated transcription factors and overall canonical functions via Ingenuity Pathway Analysis

A single report has suggested that liver-derived PANDER may play a role in hepatic insulin signaling and glucose production, as well as lipogenesis. These results implicate PANDER in the development and progression of T2D [107, 110]. This research will lead to a better understanding of how PANDER is regulated within the liver and how liver-derived PANDER impacts hepatic signaling in response to secretagogue stimulation. We will also gain valuable insight as to the potentially differing effects of 
intracellular and extracellular PANDER within the liver. The data obtained from this research will further the knowledge of how liver-derived PANDER plays a role in metabolic signaling. Elucidating the mechanism by which hepatic PANDER is regulated may provide novel therapeutics for the treatment of T2D.

\section{Innovation}

The study of PANDER over the past decade has focused primarily on its role in the context of its secretion from the endocrine pancreas. However, recent data indicates that liver-derived PANDER may play an important role in hepatic insulin signaling, lipogenesis and hepatic glucose production [67, 69, 77]. In addition, PANDER may serve as a critical cofactor in the onset and progression of T2D. Despite PANDER's suggested role within the liver, the study of the regulation of hepatic PANDER expression has not been explored. The PANDER promoter provides an excellent mechanism by which the transcriptional regulation of PANDER in the liver can be examined. Although the activation of the PANDER promoter has been studied in the endocrine pancreas and has shown activity within the liver, there have been no studies to date that explore the regulation of the PANDER promoter in the liver [66, 87]. This study seeks to identify the mechanism by which PANDER is regulated in the liver and its function in response to activation by secretagogues. 
This study will prove invaluable in further understanding how hepatic PANDER is regulated and determines glycemic levels and could further demonstrate that PANDER, under pathological conditions, may propagate T2D and represent a future therapeutic target. 


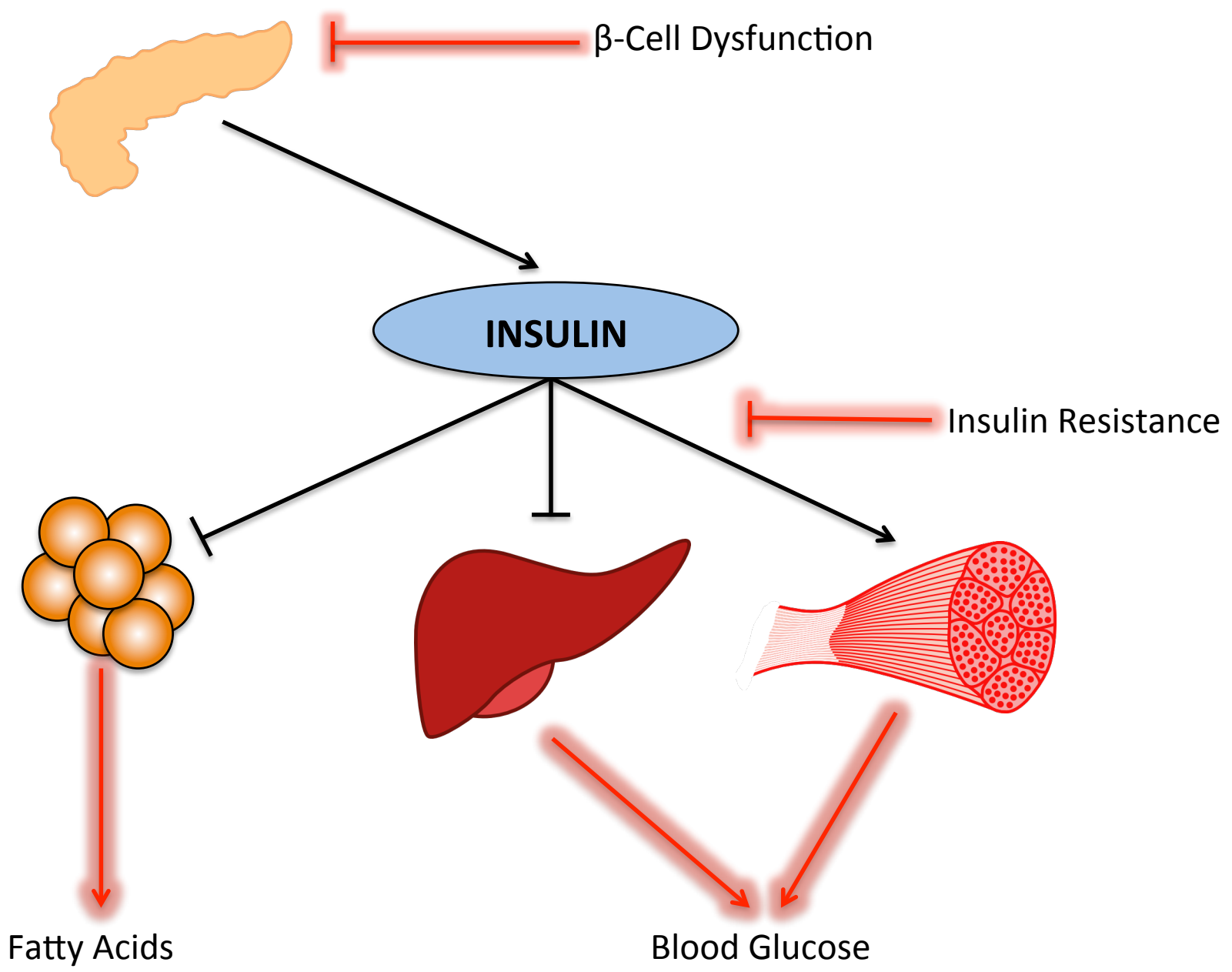

Figure 1: Pathophysiology of T2D. In healthy individuals, the pancreas secretes insulin in response to elevated blood glucose. In response to insulin, skeletal muscles uptake glucose into the cells, the glucose production within the liver is suppressed and adipose tissue is prevented from releasing free fatty acids into the blood. Under conditions of diabetes, $\beta$-cell dysfunction causes a partial loss of insulin production. Insulin resistance in the tissues results in insulin action not being completed within the cells. Both conditions result in an increase in free fatty acids and glucose within the blood. Adapted from Stumvoll, et. al. (2005) [8]. 


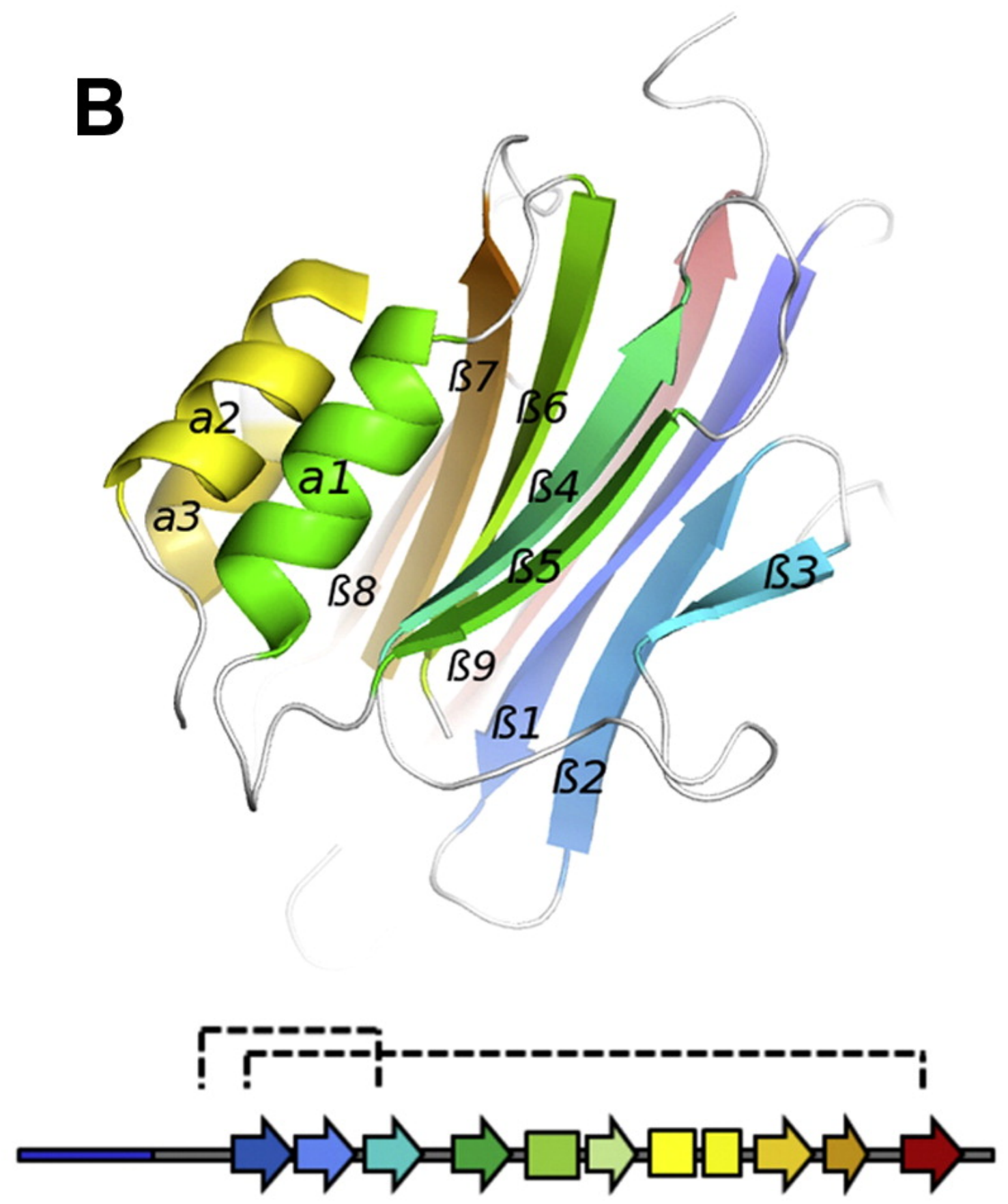

Figure 2: PANDER $\boldsymbol{\beta}-\boldsymbol{\beta}-\boldsymbol{\alpha}$ Fold. Murine PANDER structure above with diagram below colored from blue to red from $N$ terminus to $C$ terminus. Disulphide topology is indicated by dashed lines. Figure from Johansson et. al. (2013) [64] with permissions from Elsevier. 


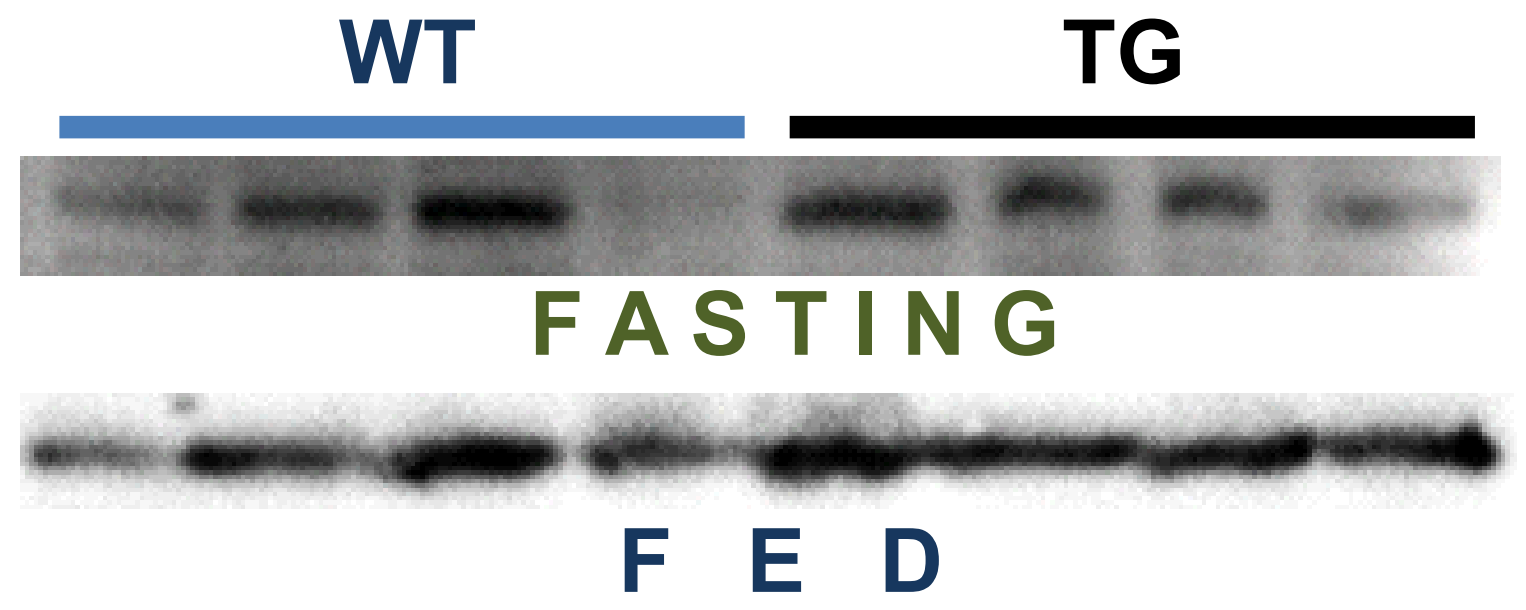

Figure 3: Increased PANDER levels during fed conditions. Western analysis was performed on liver lysates obtained from B6SJLF WT and PANTG mice following a 16 hour fast (Top) or 4 hour re-feed (Bottom). Lanes 1-4 on top and bottom western are from WT livers, and lanes 5-8 are from PANTG mice. Figure from RobertCooperman et. al. (2014) [70] with permissions from Bioscientifica Ltd. 


A
Lipid metabolism
Nucleic acid metabolism
Drug metabolism
Vitamin and mineral metabolism
Energy production
Cell morphology
Amino acid metabolism
DNA replication, recombination, and repair
Cellular function and mainlenance
Protein synthesis
Free radical scavenging
Carbohydrate metabolism
Cell death and survival
Cell-to-cell signaling and interaction
Cellular assembly and organization
Cellular compromise
Cellular growth and proliferation
Protein trafficking
Cell cycle

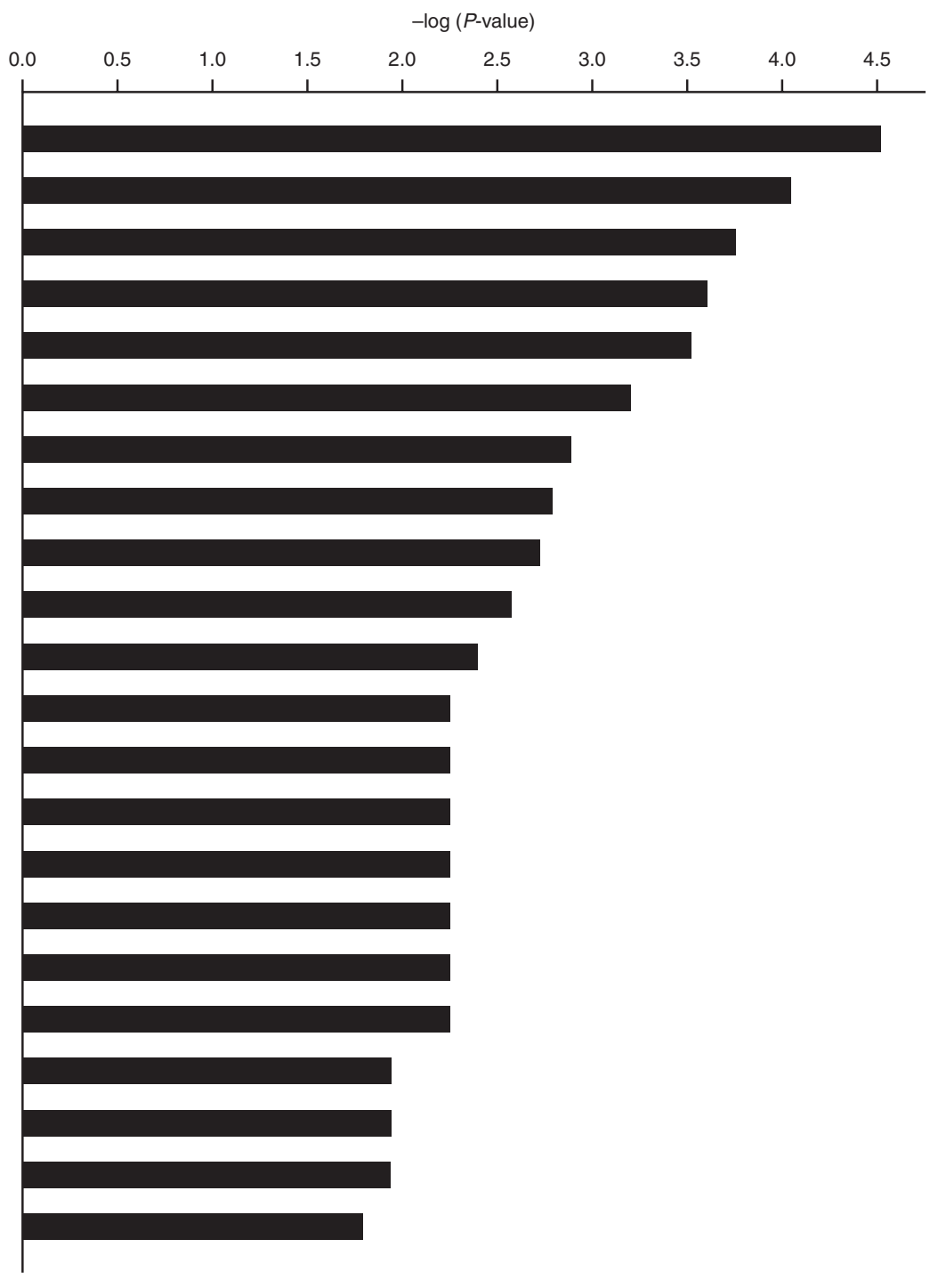

Figure 4: Stable isotope labeling of amino acids in cell culture (SILAC) analysis of PANTG. Relative changes in the levels of identified proteins were determined by the ratio of PANTG to that of WT and normalized to Tubulin B5. (A) IPA analysis of predicted impacted metabolic functions indicates lipid metabolism is altered in PANTG liver. (B) Differentially expressed hepatic proteins identified in PANTG and predicted to increase triglyceride production. (C) Network analysis of differentially expressed proteins that molecularly interact involved in triglyceride production. Figure from RobertCooperman et. al. (2014) [70] with permissions from Bioscientifica Ltd. 
B

\begin{tabular}{|c|c|}
\hline $\begin{array}{c}\text { Increased PANTG } \\
\text { hepatic proteins }\end{array}$ & $\begin{array}{c}\text { Fold change } \\
\text { above WT }\end{array}$ \\
\hline FABP1 & 2.8 \\
\hline PKA & 2.8 \\
\hline PEPCK & 3.5 \\
\hline SCP2 & 3.5 \\
\hline ACSL1 & 4.6 \\
\hline ENTPD5 & 8.3 \\
\hline
\end{tabular}

C

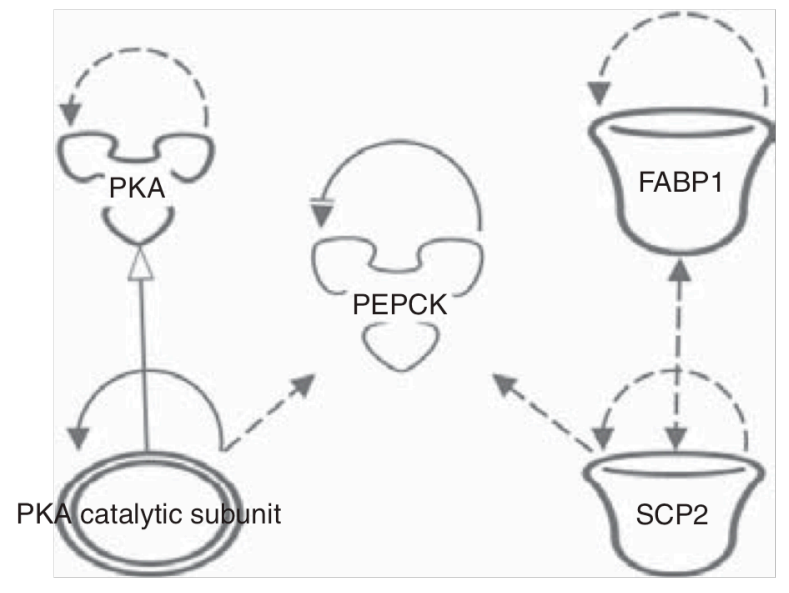

Figure 4: continued. 


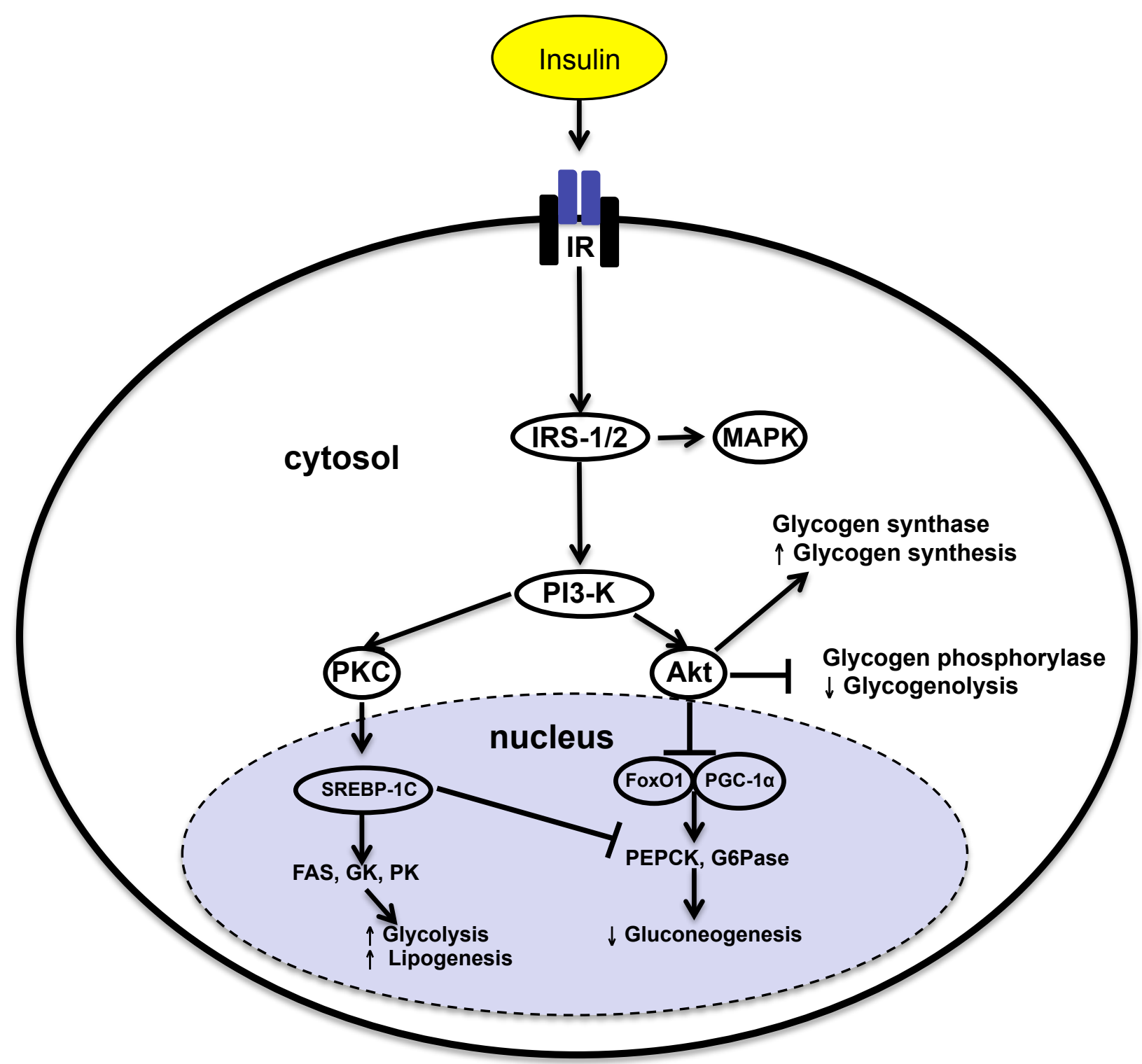

Figure 5: Metabolic signaling in the liver in response to insulin. The insulin signaling cascade is initiated by the binding of insulin to the insulin receptor. This binding induces IRS proteins to bind to the phosphorylated receptor and are subsequently phosphorylated creating docking sites for proteins such as IRS-1/2 and phosphoinositide-3 (PI-3) kinase. This signal is then transferred onto further molecules such as Akt a PKC. The overall action of insulin on the liver is to decrease gluconeogenesis and increase glycolysis, glycogen synthesis and lipogenesis. Figure adapted from Dr. Burkhardt's grant proposal. 


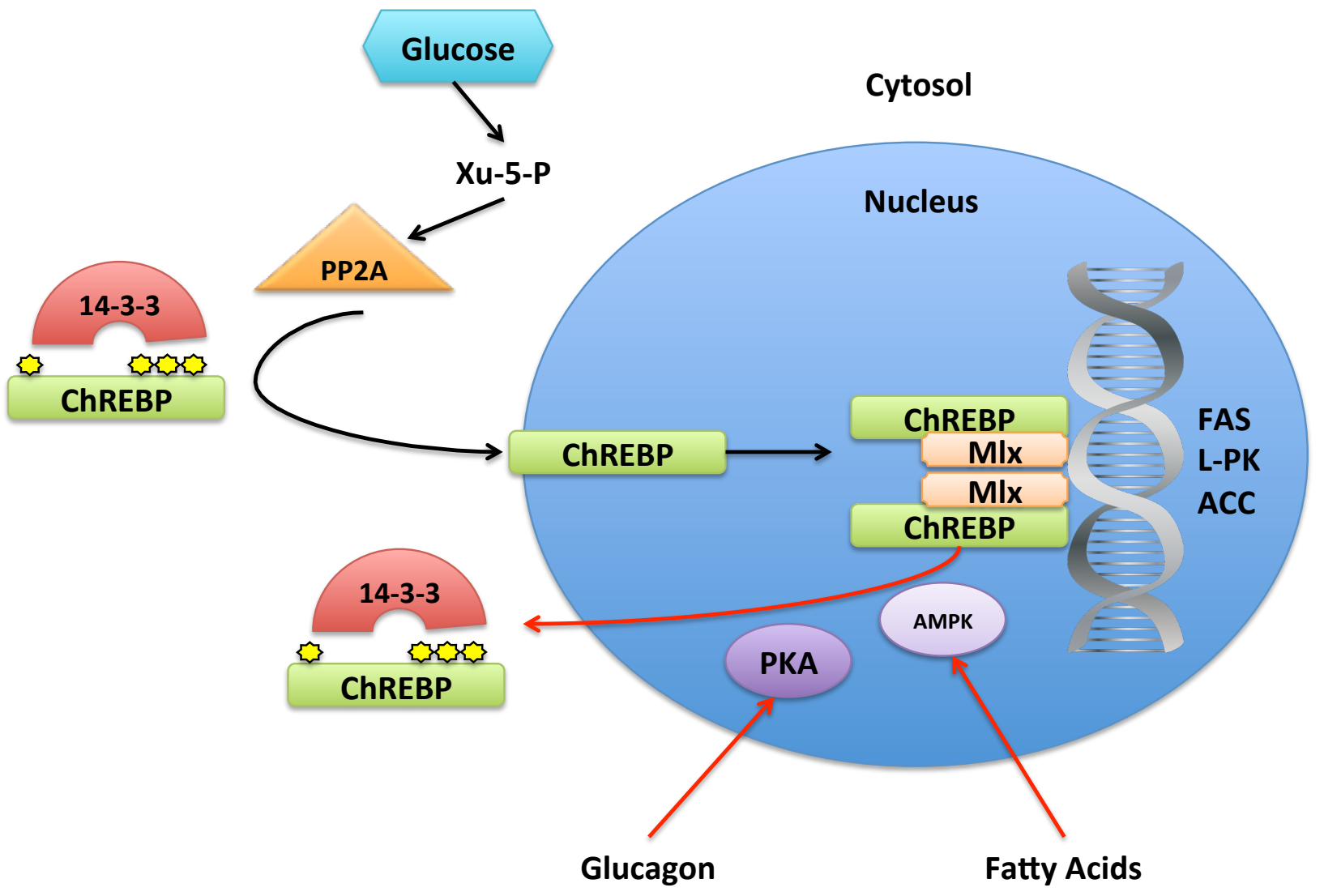

Figure 6: Regulation of ChREBP activity in the nucleus. Increased glucose levels cause PP2A activity to dephosphorylate ChREBP and facilitate its translocation to the nucleus. Nuclear ChREBP dimerizes with $\mathrm{Mlx}$ and binds to the promoters of its target genes (FAS, ACC, L-PK). This activity is reversed by the the activity of PKA and AMPK, activated by glucagon and fatty acids, which phosphorylate ChREBP and cause it to be shuttled from the nucleus. Adapted from Uyeda, et. al. (2006) [98]. 

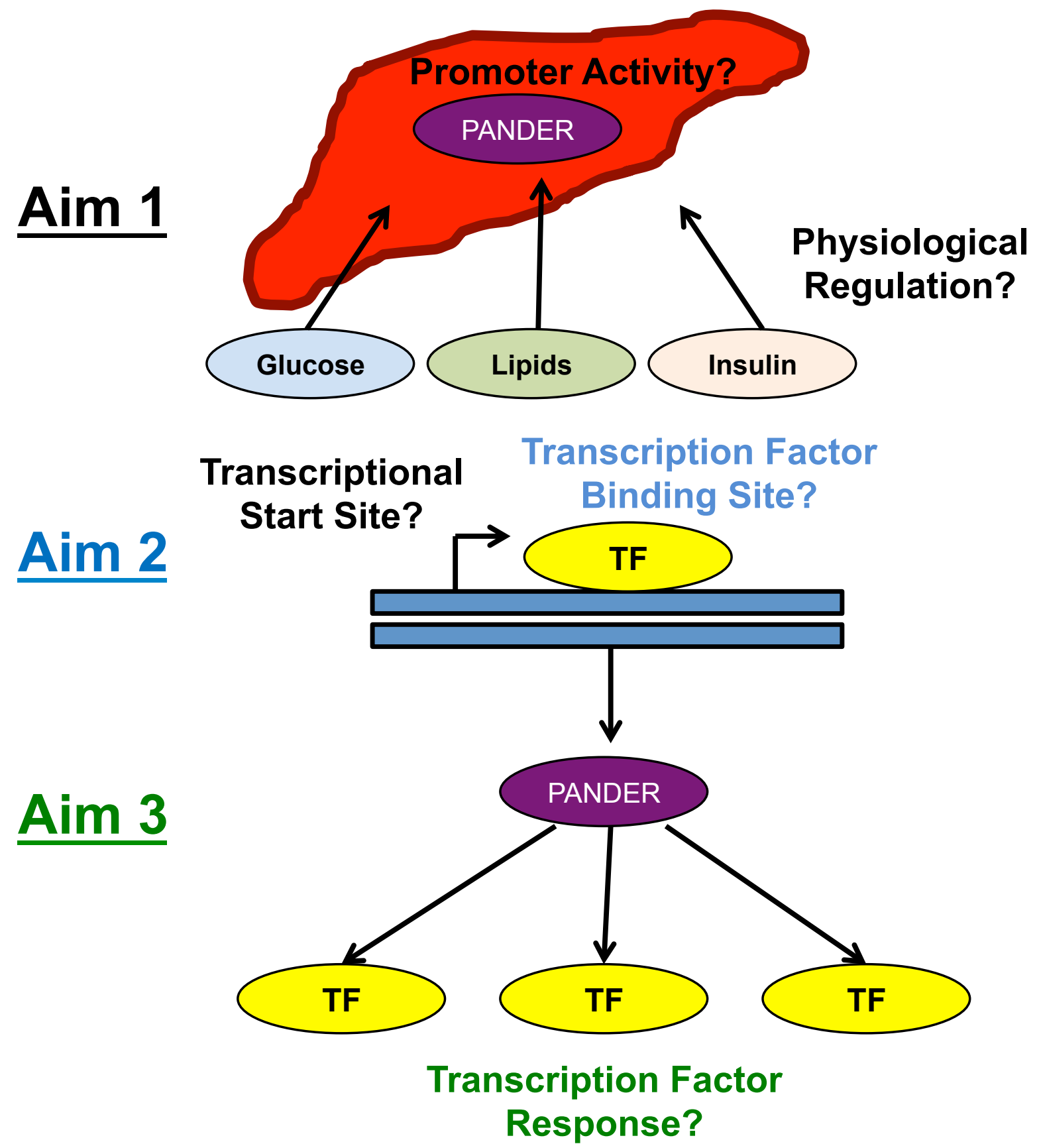

Figure 7: Schematic of Specific Aims. This study characterized the expression, regulation and function of PANDER within the liver. 


\section{CHAPTER 2: MATERIALS AND METHODS}

\section{Note to Reader}

Portions of this chapter have been previously published in Molecular and Cellular Endocrinology, 2015, 413: 101-112, and have been reproduced with permission from Elsevier. Whitney A. Ratliff designed the study, acquired, analyzed and interpreted the data and wrote the manuscript. Mark G. Athanason, Alicia C. Chechele, Melanie N. Kuehl, Amanda M. Fernandez and Catherine B. Martia assisted with data acquisition. Brant R. Burkhardt designed the study, interpreted the data and contributed to manuscript writing. All authors read and approved the final manuscript.

\section{Identification of Transcriptional start Site}

The 5'-ends of murine PANDER mRNAs were isolated by 5' RNA Ligase Mediated Rapid Amplification of CDNA Ends (RLM-RACE) using the FirstChoice RLM-RACE Kit (Ambion, Austin, TX). Unless otherwise noted, all reagents were supplied by the manufacturer. The RLM-RACE procedure is outlined in Figure 8. Total RNA was isolated separately from the BNLCL2 murine embryonic hepatocyte cell line and homogenized murine liver tissue (wild-type B6SJLF) 
using the RNeasy Mini Kit (Qiagen, Hilden, Germany). All RNA was treated with RNAse-free DNAse (Qiagen) to remove any potential contaminating genomic DNA. Approximately $10 \mu \mathrm{g}$ of RNA from the BNLCL2 cell line and murine liver tissue were treated by calf intestinal phosphatase to remove 5'-phosphates from DNA, rRNA, tRNA, and any degraded mRNA. The RNA was then purified by phenol-chloroform extraction, and treated with tobacco acid pyrophosphatase to remove the cap structure from intact mRNAs. A 45-base RNA adapter oligonucleotide was then ligated to the previously decapped mRNA population using T4 RNA ligase. A random primed reverse transcription polymerase chain reaction (RT-PCR) was performed using random decamers. Sequences of all listed primer pairs used in the subsequent experiments were detailed previously [111]. Following the reverse transcription reaction, a nested $\mathrm{PCR}$ of 35 cycles $\left(94^{\circ} \mathrm{C}-30 \mathrm{~s} ; 60^{\circ} \mathrm{C}-30 \mathrm{~s}\right.$; and $\left.72^{\circ} \mathrm{C} \quad 30 \mathrm{~s}\right)$ was performed using first round primers that contained the 5' RACE outer adapter specific primer (5' RACE Outer) along with a PANDER specific outer primer (PANDER74R). A second round of amplification was performed using $2 \mu l$ of the first round reaction products under the same conditions with the exception of the 5' RACE inner adapter specific primer (5' RACE Inner) and an inner PANDER specific primer (PanProTR+8TA or $+491)$ and the PCR annealing temperature was increased to $62^{\circ} \mathrm{C}$. The resultant PCR products were subsequently cloned into the 
pCR2.1 cloning vector (Invitrogen, Carlsbad, CA). Plasmids were then sent to the Moffitt Cancer Center Molecular Genomics Core (Tampa, FL) for sequencing to identify the precise TSS.

\section{Identification of Putative Transcriptional Factor Binding Sites}

Potential transcriptional binding sites located within the -832 to -3 (relative to previously identified pancreatic transcriptional start site) region of the PANDER promoter were identified using the program of MatInspector 8.0 (http://wWw.genomatix.com) or visually identified.

\section{Western Analysis of PANDER Expression}

A commercially prepared tissue blot (Zyagen, San Diego, CA) was used containing the following murine tissues: Brain (whole), stomach (whole), intestine (whole), colon, liver, lung, kidney, heart, ovary, skeletal muscle, spleen, testis, thymus, uterus (non-pregnant) and placenta (late pregnancy). The blot was rehydrated according to manufacturer's instructions and probed for PANDER using the FAM3B M-80 rabbit polyclonal antibody (Santa Cruz Biotechnology, Santa Cruz, CA) at a 1:1000 dilution in commercial StartBlock ${ }^{\mathrm{TM}}$ blocking buffer (Thermo Scientific, Waltham, MA). The blot was then incubated in goat anti-rabbit secondary antibody (Santa Cruz Biotechnology) at a 1:10,000 dilution. The blot was developed using ECL Western Blotting 
Substrate (Pierce, Rockford, IL) and visualized using the LAS 3000 Intelligent Dark Box (Fujifilm, Stamford, CT) .

\section{Construction of the PANDER Promoter Luciferase Constructs}

Initial PANDER/luciferase constructs were created as described previously [111]. Further constructs were created utilizing a Bacterial Artificial Chromosome (BAC) construct containing 14 kb of sequence upstream of the PANDER coding region as a PCR template. This BAC was obtained from Dr. Brant Burkhardt and was used as the template for all previously created PANDER promoter constructs [111]. Various regions of the PANDER promoter spanning from $193 \mathrm{bp}$ upstream of the hepatic transcriptional start site to 8 bp downstream of the translational start codon (+34 relative to the hepatic transcriptional start site) were PCR amplified and cloned into the pGL3-basic (Promega, Madison, WI) luciferase reporter plasmid. The PCR amplification introduced flanking KpnI and MIuI sites that were utilized for subsequent ligation with T4 DNA ligase (Invitrogen, Carlsbad, CA) into the dephosphorylated and restriction digested pGL3basic plasmid. Primers utilized for the promoter truncations are listed in Table 1. All primers were synthesized by Integrated DNA Technologies (Coralville, IA). All PANDER/luciferase constructs were confirmed by restriction digestion. Plasmid purifications were performed using the QIAprep spin Miniprep kit 
(Qiagen, Hilden, Germany) and confirmed via gel electrophoresis. All construct locations are relative to the identified hepatic transcriptional start site or translational start codon (where noted). All utilized promoter constructs are detailed in Figure 9.

\section{Cell Culture and Transient Transfections}

$\beta$-TC3 (murine insulinoma) cells were cultured in complete RPMI1640 (11 mmol/l glucose) with 10\% heat-inactivated fetal bovine serum, 100 units/ml penicillin, $100 \mathrm{\mu g} / \mathrm{ml}$ streptomycin, and 2 mmol/l l-glutamine at $37{ }^{\circ} \mathrm{C}, 5 \frac{\circ}{\mathrm{CO}_{2}}$. BNLCL2 (murine embryonic hepatocyte), C2C12 (murine myoblast), NIH-3T3 (murine fibroblast) and HepG2 (human hepatocellular carcimoma) cells were cultured in complete Dulbecco's modified Eagle's medium (DMEM) supplemented with the same reagents listed above. Prior to luciferase experiments, transfection efficiency was confirmed visually through transfection of the pEGFP-C1 plasmid (Figure 10). For each transfection, approximately $2 \times 10^{5}$ cells per well were plated in a tissue culture treated 24-well dish (BectonDickinson, Heidelberg, Germany). PANDER/luciferase constructs were transfected using Lipofectamine 2000 (Invitrogen, Carlsbad, CA). Transfections of each plasmid were performed in triplicate in at least two independent experiments. Two hours post transfection, the media was removed and replaced with fresh 
culture media. Eighteen hours post-transfection, cells were washed with PBS and lysed in $100 \mu l$ of $1 X$ Glo Lysis Buffer (Promega). Luciferase activity of the promoter constructs was measured sequentially using the Dual-Luciferase Reporter Assay System (Promega). Luciferase activity was measured for $10 \mathrm{~s}$ after a 2-s delay using a Monolight 3010 luminometer (Analytical Luminescence Laboratory, San Diego, CA).

\section{Insulin and Palmitate Stimulation}

To determine whether the murine PANDER promoter was responsive to insulin and/or palmitate, BNLCL2 and/or HepG2 cells were plated in 24-well tissue culture dish in appropriate media (DMEM) containing 10\% heat-inactivated fetal bovine serum, 100 units/ml penicillin, $100 \mathrm{\mu g} / \mathrm{ml}$ streptomycin, and $2 \mathrm{mmol} / \mathrm{l}$ lglutamine. As described previously, cells were plated at $2 \times 10^{5}$ cells per well. After 24 h, media was replaced with glucose-free DMEM containing $1 \%$ FBS and 2 mmol/11-glutamine in the complete absence of penicillin and streptomycin. The FBS was reduced to 1\% to prevent additional nutrients (ie. Insulin or palmitate) being introduced into the media. Penicillin and streptomycin were not added due to cytotoxic effects when employed in conjunction with the transfection reagent of Lipofectamine 2000 (Invitrogen, Carlsbad, CA). After $4 \mathrm{~h}$, the cells were

transfected with PANDER/luciferase constructs using 
Lipofectamine 2000, as described previously. After $2 \mathrm{~h}$, the glucose-free media was removed and replaced with appropriate media containing various increasing insulin (Humulin R, Eli Lilly, Basingstroke, UK) or palmitate (Sigma-Aldrich, St. Louis, MO) concentrations. Palmitate was dissolved in 95\% ethanol and an ethanol-only control was included to account for cell toxicity related to ethanol treatment. This ethanol-only control contained $0.95 \%$ ethanol, the equivalent of the ethanol contained in the highest palmitate treatment. Additional combination treatment of palmitate and glucose (Fisher Chemicals, Pittsburgh, PA) was included as well. The FBS concentration was maintained at 1\%, and no penicillin/streptomycin was added. After addition of the insulin-containing or palmitate-containing media, cells were incubated for an additional $18 \mathrm{~h}$ and then harvested for luciferase expression as described above. Each insulin/palmitate condition was evaluated in triplicate from 3 independent experiments.

\section{Palmitate Stimulation of $\beta-T C 3$ Cell Line}

PANDER promoter activity was evaluated in the $\beta$-TC3 pancreatic $\beta$-cell line in response to stimulation with palmitate. $\beta$-TC3 cells were cultured and luciferase assays were performed as stated above using the $-832 /-3$ PANDER promoter construct. In addition, a time course was performed to evaluate relative 
luciferase levels over time at 6,12 and 18 hrs post treatment. An ethanol-only control was performed to rule out potential cytotoxic effects of the ethanol contained within the palmitate treatments.

\section{Glucose-Responsive Experiments}

To determine the potential glucose-responsive expression of the murine PANDER promoter, the above procedure was followed using varying concentrations of glucose. Initial experiments were performed using 1\% FBS to prevent additional unaccounted glucose being introduced into the media from the typical 10\% FBS concentration which contributes to about $0.5 \mathrm{~mm}$ glucose to the final glucose concentration. To further abrogate the potential effects of residual glucose in 1\% FBS, subsequent experiments were performed using $0.1 \% \mathrm{FBS}$.

\section{RT-PCR}

To assess the levels of hepatic PANDER mRNA under glucosestimulation, primary murine hepatocytes (Triangle Research Labs, Triangle Park, NC) were plated on a 6-well dish at a concentration of $1.2 \times 10^{6}$ cells/well using the cryopreserved Animal Hepatocyte Thawing and Plating Medium provided (Triangle Research Labs). The following day, media was removed and replaced with fresh media containing $0,5.5,11$ or $22 \mathrm{mM}$ 
glucose. After 18 hours, these cells were harvested and RNA was extracted using the RNeasy Mini Kit (Qiagen, Hilden, Germany), all lysates were run through gDNA eliminator spin columns, provided in the kit, to remove any genomic DNA contamination. RT-PCR was performed according to manufacturer instructions using the TaqMan $^{\circledR}$ RNA-to- ${ }_{T^{T M}}$ 1-Step Kit (Catalog \#: 4392653) and the Fam3B Gene Expression Assay (Assay ID: Mm00508056_m1) with the $\beta$-actin Gene Expression Assay as a control (Assay ID: Mm00607939_s1) from Life Technologies (Carlsbad, CA). Real Time PCR was run using the Applied Biosystems step One Plus Real Time PCR System (Life Technologies, Carlsbad, CA). Relative levels of mRNA expression were normalized to $\beta$-actin and calculated using the $2^{-{ }_{\triangle \Lambda} \mathrm{CT}}$ method from 2 independent experiments with each condition examined in duplicate.

\section{ChREBP Co-Transfection Experiments}

To determine the potential activation of the PANDER promoter by the transcription factor ChREBP, we obtained several expression constructs used in previous experiments by Dr. Howard Towle (University of Minnesota, Minneapolis, MN) [112-117]. We utilized an expression construct for wild-type ChREBP protein as well as Mlx, the co-factor shown previously to facilitate ChREBP activity [114]. An acetyl co-A carboxylase promoter construct was used as a positive control to assess the activity of these 
expression plasmids (Figure 11) [117]. To assess the effect of ChREBP on the PANDER promoter under baseline conditions (10\% FBS), we followed the original protocol listed above and transfected the cells with the $-832 /-3$ PANDER promoter construct alone or with the addition of the Mlx construct, the ChREBP construct or both. Additional experiments were performed under glucose-stimulatory conditions using the procedure outlined above for glucose-stimulation in DMEM containing 1\% FBS.

\section{Chromatin Immunoprecipitation}

Chromatin immunoprecipitation was performed using the EZ-ChIP (Millipore, Darmstadt, Germany) kit. Manufacturer's instructions were followed unless otherwise noted. Cryopreserved primary murine hepatocytes (Triangle Research Labs, Research Triangle Park, NC) were plated on a 6-well dish at a concentration of 1.2 $\times 10^{6}$ cells/well using the Cryopreserved Animal Hepatocyte Thawing and Plating Medium provided (Triangle Research Labs). The next day, prior media was removed and replaced with fresh media containing 11 or $22 \mathrm{mM}$ glucose. These cells incubated overnight and were then fixed with $1 \%(\mathrm{v} / \mathrm{V})$ fresh formaldehyde to cross-link the DNA with any associated transcription factors. After 10 mins incubation, $300 \mu \mathrm{L} 10 \mathrm{x}$ glycine was added to quench the unreacted formaldehyde. The cells were then harvested according to manufacturer's protocol and sonicated using the 
Bioruptor Plus sonicator (Diagenode, Denville, NJ) for 10 mins on HIGH with 30 sec ON/OFF cycles. For immunoprecipitation, the following antibodies were added to one of two tubes: $1.0 \mu g$ normal mouse IgG (Millipore, Darmstadt, Germany) and $10 \mu g$ ChIP grade rabbit polyclonal anti-ChREBP antibody (Abcam, Cambridge, MA) .

\section{PCR of Immunoprecipitated Chromatin}

Following the purification of immunoprecipitated chromatin, PCR was performed on the samples. Previously designed primers were used to amplify portions of the PANDER promoter, including $293 /+25,-193 /+25$ and $-93 /+25$. Two sets of tubes were prepared for PCR. One contained water, Accuprime Supermix II (Invitrogen, Carlsbad, CA), appropriate primers and immunoprecipitated DNA. The second set contained input DNA in place of the immunoprecipitated DNA. All PCR reagents were added to thinwalled, flat-topped, $0.5 \mathrm{~mL}$ PCR tubes (Molecular Bioproducts, San Diego, CA). PCR was run in an MJ Research PTC-200 DNA Engine thermocycler using the following conditions: $94{ }^{\circ} \mathrm{C}$ for $3 \mathrm{~min}$ (one cycle, for initial denaturation), $94^{\circ} \mathrm{C}$ for $20 \mathrm{~s}$ (denaturation)/59 ${ }^{\circ} \mathrm{C}$ for $30 \mathrm{~S}$ (annealing)/72 ${ }^{\circ} \mathrm{C}$ for $30 \mathrm{~S}$ (extension) (32 cycles), with one cycle of $72^{\circ} \mathrm{C}$ for 2 min for final extension. Samples were then analyzed on a 1\% agarose gel 
and visualized using the Amersham Imager 600 (GE Healthcare Life Sciences, Pittsburgh, PA).

\section{Site-Directed Mutagenesis}

Site directed mutagenesis was performed to create a PANDER promoter construct not containing the third E-box element. The PANDER promoter luciferase construct containing the $-493 /-3$ region of the PANDER promoter was mutated using the Agilent Quikchange II Site-Directed Mutagenesis kit (Santa Clara, CA) according to manufacturer instructions. A single primer was used that encompassed the regions adjacent, but not containing the 6 nucleotides (CATTTG) that make up the third E-box element. The primer used to create the mutant construct is listed in Table 1. All primers were synthesized by Integrated DNA Technologies (Coralville, IA). Plasmid purifications were performed using the QIAprep Spin Miniprep kit (Qiagen, Hilden, Germany) and confirmed via gel electrophoresis. The PANDER/luciferase construct deletion was confirmed by sequencing at the Moffitt Sequencing Core (Tampa, FL). Subsequent luciferase assays were performed using the procedures detailed previously for baseline activity and glucose-responsiveness. 
RT-PCR of PANDER Levels in Livers from Non-Obese Diabetic (NOD) Mice

To determine differences in PANDER mRNA levels in a Type I Diabetes in vivo model, several NOD mouse livers were provided from Dr. Mark Atkinson of the University of Florida. All livers were obtained from 28-week-old females housed in compliance with the University of Florida Institutional Animal Care and Use Committee (IACUC). Blood glucose readings were monitored weekly using a Freestyle Blood Glucose monitoring system (Abbott, Abbott Park, IL) to assess the progression of diabetes. Prior to sacrifice, 3 of 7 NOD mice were determined to be within the diabetic range (consecutive blood glucose readings $>200 \mathrm{mg} / \mathrm{dL}$ ), while the remaining 4 were non-diabetic. Livers were obtained upon sacrifice and snap-frozen in liquid nitrogen prior to being stored at $-80^{\circ} \mathrm{C}$. Livers were thawed and homogenized using a Qiagen TissueRuptor Tissue Disruption Systems and RNA was isolated according to manufacturer instructions using the RNeasy Mini Kit (Qiagen, Hilden, Germany). RT-PCR was performed on RNA samples, as detailed previously, using 500 ng NOD liver RNA per sample.

\section{Statistical Analysis}

Data are presented as mean \pm S.E.M. Statistical significance of differences between groups was analyzed by unpaired student's t- 
test or by one-way analysis of variance (ANOVA) when more than two groups were compared. 


\section{RLM-RACE}

RNA extraction from desired cells or tissues, treat with DNase

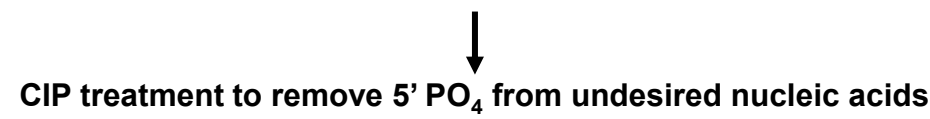

(intact mRNA is not altered)

Phenol-Chloroform extraction

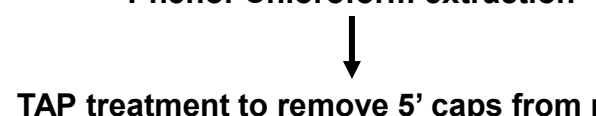

TAP treatment to remove 5' caps from mRNA

$\downarrow$

Ligation 5' RACE adapter to 5' end of decapped mRNA

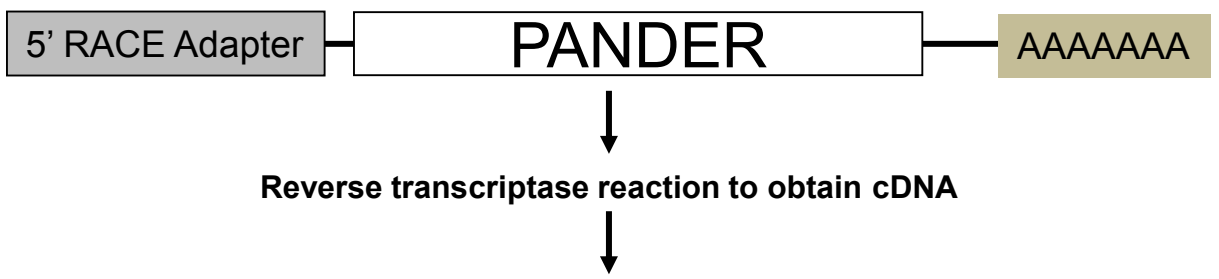

First round PCR using outer RACE and PANDER primers

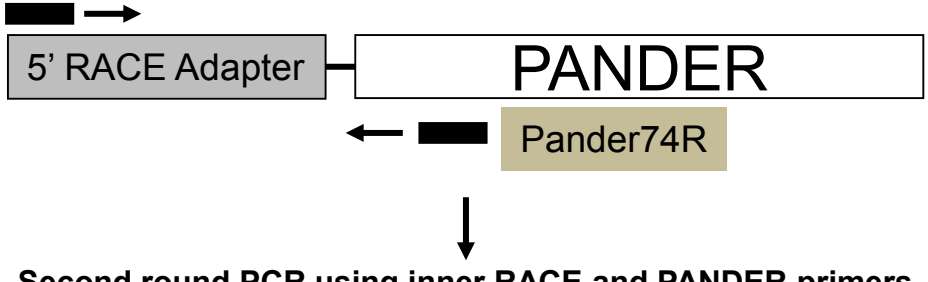

Second round PCR using inner RACE and PANDER primers

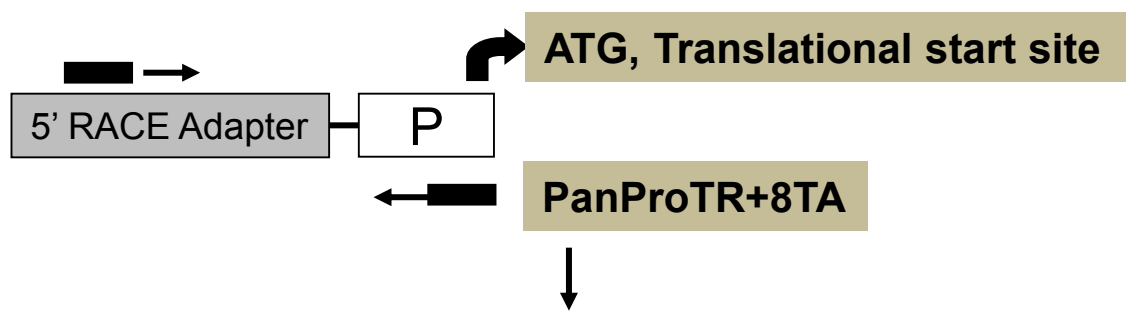

TA cloning into pCR2.1 vector

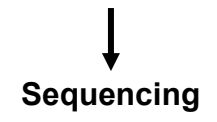

Figure 8: RLM-RACE procedure. This scheme outlines the steps in the RLM-RACE procedure seeking to identify the transcriptional start site of the PANDER promoter. 


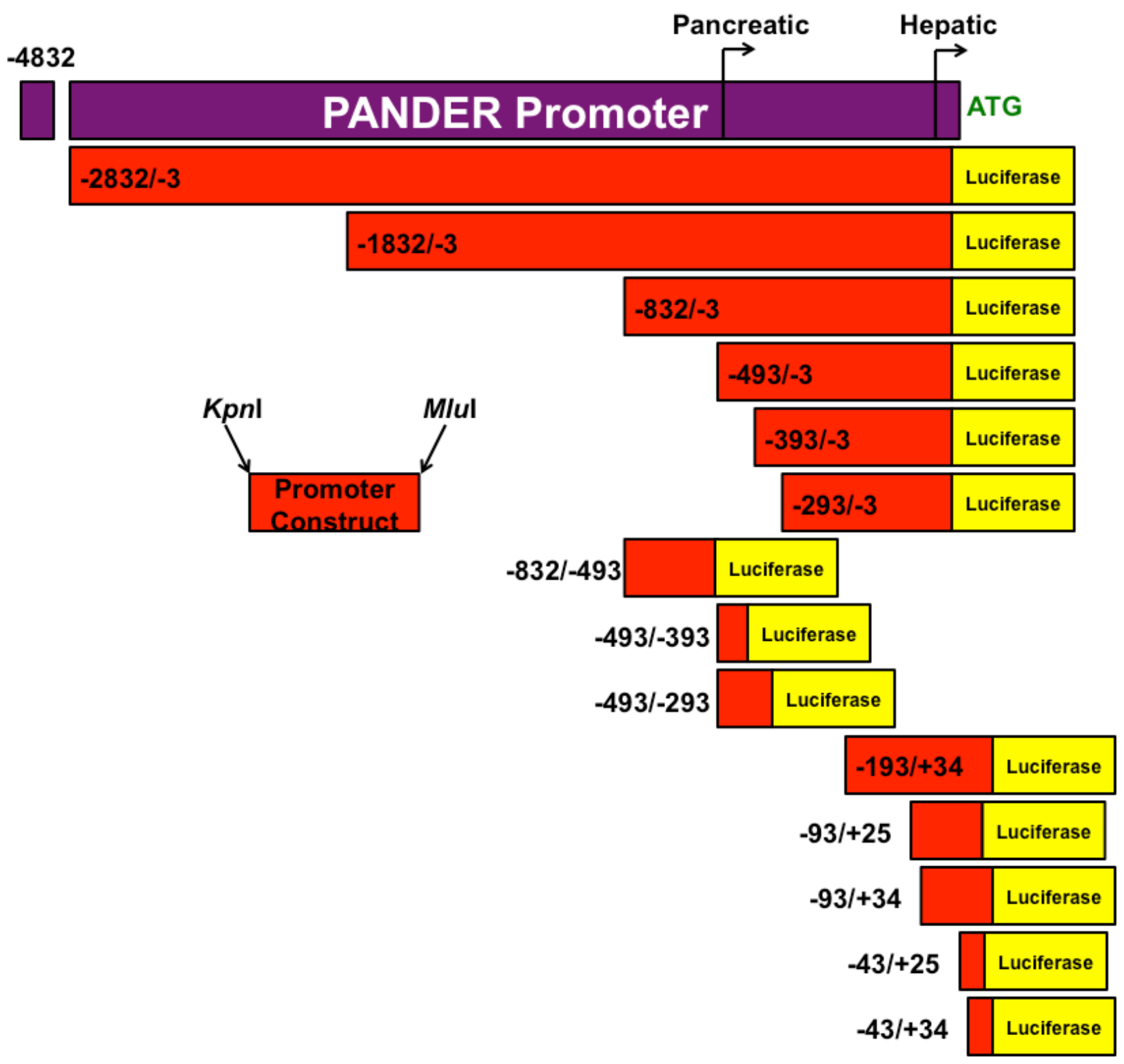

Figure 9: PANDER promoter luciferase constructs. Several PANDER promoter constructs were created. Fragments of the PANDER promoter were flanked by KpnI and MIuI restriction sites to allow for cloning into the pGL3-basic luciferase plasmid construct. 
Table 1: List of primers utilized for creation of PANDER/luciferase constructs

\begin{tabular}{|c|c|c|}
\hline Name & Sequence & $\begin{array}{l}\text { Forward } \\
\text { Reverse }\end{array}$ \\
\hline PanPro+300KpnIF & 5'-GCGGTACCCTGTGCAGCCCCACCCAT-3' & $\mathrm{F}$ \\
\hline PanPro+400KpnIF & 5'-GCGGTACCTTTTCTGCCTGCCCCAAG-3' & $\mathrm{F}$ \\
\hline PanPro+450KpnIF & 5'-GCGGTACCTAGGCCAGCTTTCATAGG-3' & $\mathrm{F}$ \\
\hline PANDERTA+8Mlul & 5'-GCACGCGTGGACGCATCTTCCAGAAACTGCT-3' & $\mathrm{R}$ \\
\hline PANPRO-1TAMlul & 5'-GCACGCGTCTTCCAGAAACTGCTCTCTTTC-3' & $\mathrm{R}$ \\
\hline PanProDelEbox3 & 5'-CTTTATGGATCCTCCCTGGGAATTGGTCCAC-3' & $\mathrm{F}$ \\
\hline
\end{tabular}




\section{Transfection Efficiency}
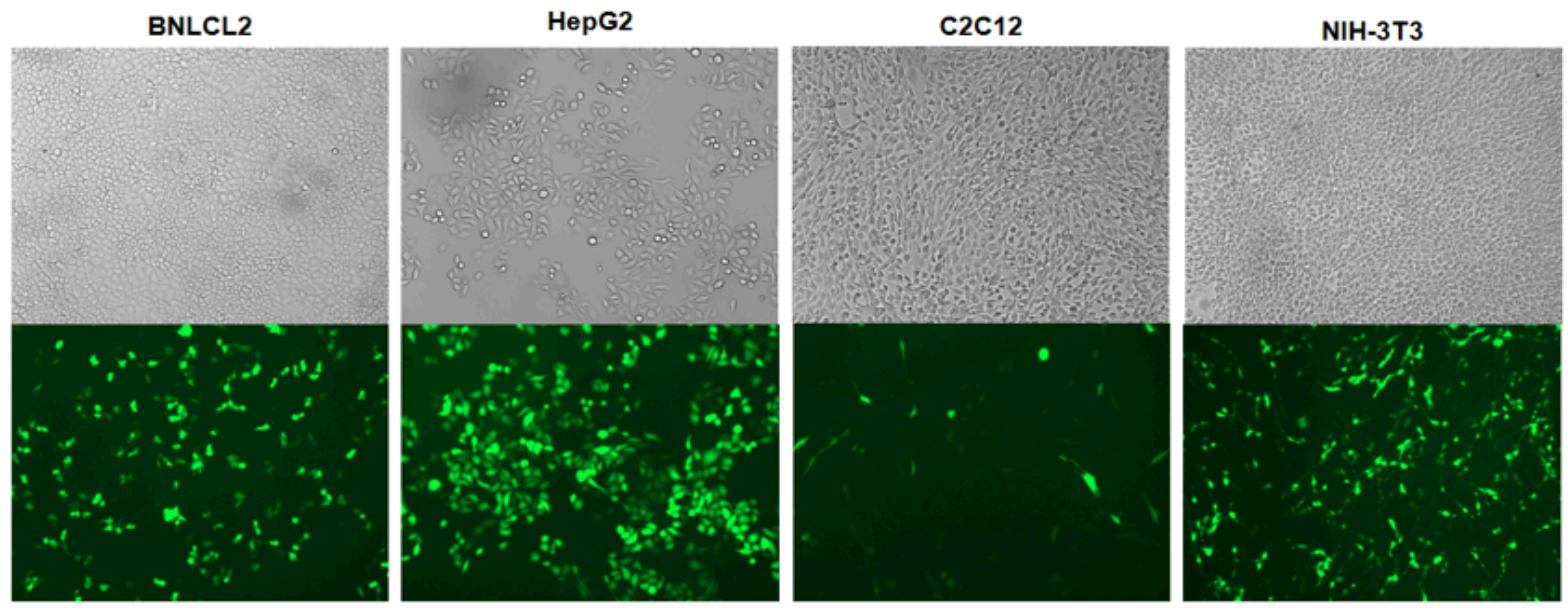

Figure 10: Transfection Efficiency of BNLCL2, HepG2, C2C12 and NIH-3T3 cell lines. BNLCL2, HepG2, C2C12, and NIH-3T3 cell lines were transfected with the pEGFP-C1 plasmid construct and evaluated for transfection efficiency via fluorescent microscopy . 


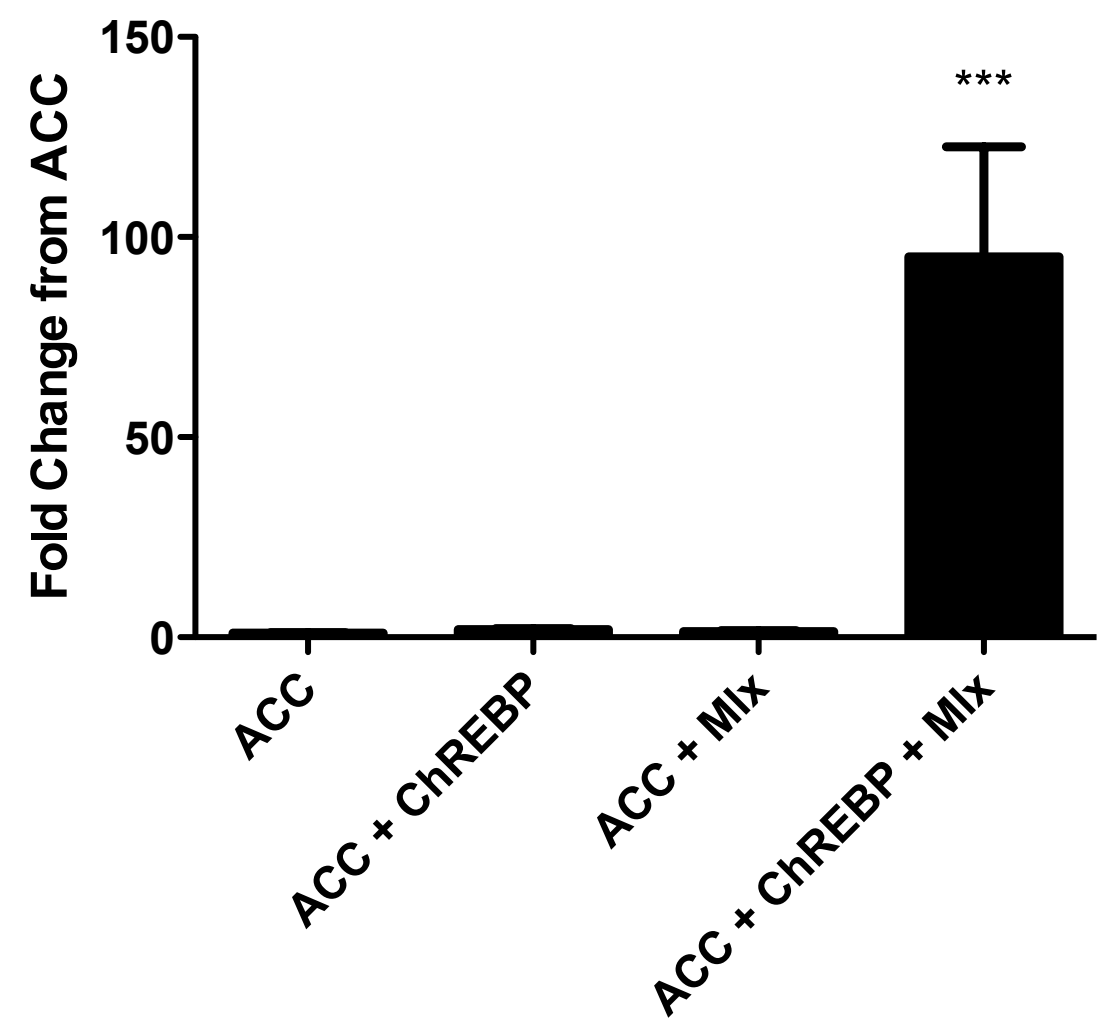

Figure 11: Acetyl-CoA Carboxylase promoter luciferase control plasmid activity, co-transfected with ChREBP and Mlx expression plasmids. ChREBP and Mlx expression plasmid activity was confirmed using a ACC promoter luciferase construct. ACC promoter is only active when ChREBP and $\mathrm{Mlx}$ expression plasmids are present. 


\section{CHAPTER 3: RESULTS}

\section{Note to Reader}

Portions of this chapter have been previously published in Molecular and Cellular Endocrinology, 2015, 413: 101-112, and have been reproduced with permission from Elsevier. Whitney A. Ratliff designed the study, acquired, analyzed and interpreted the data and wrote the manuscript. Mark G. Athanason, Alicia C. Chechele, Melanie N. Kuehl, Amanda M. Fernandez and Catherine B. Marelia assisted with data acquisition. Brant R. Burkhardt designed the study, interpreted the data and contributed to manuscript writing. All authors read and approved the final manuscript.

\section{Identification of the Murine Hepatic PANDER Transcription Start}

\section{Site}

Earlier experiments evaluating PANDER transcriptional control strongly suggested the presence of an additional TSS downstream of the one previously identified [118]. To determine the presence of a unique hepatic TSS, RLM-RACE was performed on total RNA isolated from BNLCL2 murine embryonic hepatocytes and murine liver lysate (B6SJLF strain). A robust product of 
approximately 70 bp was amplified from both of these sources following PCR amplification of RLM-RACE obtained template (Figure 12A). The PCR products were subsequently cloned and sequenced (Moffitt Cancer Center), revealing a newly identified single TSS 26 bp upstream of the murine PANDER translational start codon. This is in contrast to the previously identified transcriptional start site 520 bp upstream of the translational start codon that was determined from the pancreatic $\beta$-TC3 cell line [111]. The region upstream of this newly identified TSS contains several potential glucose-responsive binding domains including three E-box elements within the $-832 /-3$ region that were later investigated for interaction with ChREBP-1 and promoter activity (Figure 12B). Figure 12C details the 2 alternatively spliced mRNA variants of the murine PANDER gene, which differ by a truncation of the $5^{\prime}$ end. It is worth nothing that the first mRNA is the gene variant investigated in this study. There have been no previous studies that have determined any expression or function of the second variant.

\section{Cell-Specific Characterization of the PANDER Promoter}

To evaluate if the PANDER promoter was tissue selective with regard to the liver specific TSS, we performed reporter gene analysis encompassing a broad range of tissue cell lines. Several truncations of the PANDER promoter, ranging from -1832 
to -3 have been previously cloned into the pGL3 luciferase vector [111]. Several cell lines were transfected evaluating these PANDER promoter plasmids to examine PANDER promoter activity with a focus on liver-derived cell lines. Significantly higher PANDER promoter mediated expression was revealed in the liver-derived cell lines of BNLCL2 murine embryonic hepatocyte cell line and the HepG2 human hepatocarcinoma cell line (Figure 13A). Lowest expression was found in the C2C12 murine myoblast cell line and NIH-3T3 murine fibroblast cell lines. Modest PANDER expression was observed in the $\beta$-TC3 murine insulinoma cell line. Based on the promoter deletion analysis, the regions encompassing $-832 /-3$ and $-493 /-3$ demonstrated the highest activity in the BNLCL2 (10.1 and 7.9-fold, respectively) and HepG2 (7.1 and 6.9-fold, respectively) cell lines above the basic vector with no significant differences between the two constructs. To further examine PANDER expression, we performed western blot analysis on a survey of murine tissues obtained from the C57BL/6J mouse. PANDER was strongly expressed in the liver followed by the brain and ovary (Figure 13B). Taken together, the PANDER promoter has robust tissue specificity in both human and murine liver-derived cell lines and this finding appears to drive PANDER protein expression within the liver. 


\section{Minimal Element of PANDER Promoter}

To identify the minimal element necessary for PANDER activity within the liver, progressive deletions of the promoter region within the $-832 /-3$ region were utilized followed by subsequent transfection into the BNLCL2 cell line. Under basal glucose conditions, the $-293 /-3$ region displayed the highest promoter activity (17.4-fold) followed by the -393/+491 (13.9-fold), 493/-393 (4.7-fold), -493/-293 (4.6-fold) and $-338 /-493$ (1.7fold) constructs (Figure 14A). Further promoter deletions were generated to identify minimal element. A 100 bp deletion between the regions of -193 to -93 resulted in a major abrogation of promoter activity (Figure 14B), with subsequent deletions not having any dramatic impact on expression.

\section{Secretagogue-Mediated Expression of the Murine PANDER Promoter in BNLCL2 Cells}

PANDER expression has been shown to be induced by glucose in pancreatic $\beta$-cells ( $\beta$-TC3), but not in $\alpha$-cells [111]. Arginine and, to a greater extent, insulin have been shown to induce PANDER expression in $\alpha$-cells, but not in $\beta$-cells [83]. In addition, in-vivo studies performed on the PANDER transgenic mouse model have indicated that PANDER expression is increased in the liver during fed conditions [70], whereas Li et al. has indicated that high fat diet may induce hepatic PANDER 
expression [67]. These studies suggest that PANDER expression may be increased in response to glucose, insulin, and/or fatty acids; however, to date, there have not been any definitive studies investigating the impact of secretagogues on PANDER promoter activity within the liver. To evaluate this activity, we transiently transfected BNLCL2 cells with the $-832 /-3$ construct in the absence or presence of varying concentrations of insulin, palmitate and/or glucose and measured promoter activity. Both insulin and palmitate were shown to significantly decrease PANDER promoter activity (Figures 15A and 15C). Combining glucose with palmitate treatment resulted in a slight increase in activity over palmitate alone, though the net result was still a decrease in activity with respect to no treatment (Figure 15D). The most potent stimulatory effect was found with glucose. Glucose treatment significantly increased PANDER promoter activity at both the 5.5 and $22 \mathrm{mM}$ glucose conditions (Figure 17A) without any measurable effect by L-glucose.

\section{Palmitate Stimulation of $\beta-T C 3$ Cell Line}

In our time course experiment, relative luciferase levels increased over time at the 6, 12 and 18 hour time points, however, overall trends remained the same. Expression increased significantly at the 6 hour time point, but there was no significant change between the 12 and 18 hour time points, 
suggesting that by 12 hours post-treatment, maximal expression has been achieved, but remains stable. PANDER promoter activity was significantly decreased in response to palmitate in a dose dependent manner, dropping approximately 60\% from the $0 \mathrm{mM}$ treatment to the $0.25 \mathrm{mM}$ treatment (Figure 16A). This did not appear to be related to a cytotoxic effect of the ethanol in the palmitate treatment, as promoter activity was not significantly decreased when the cells were treated with $0.95 \%$ ethanol when compared to no treatment (normal culture media) (Figure 16B).

\section{Minimal Element for Glucose-Responsiveness of the PANDER Promoter}

To precisely map and identify the glucose-responsive regions of the PANDER promoter, BNLCL2 cells were transfected with several truncated versions (-393 to -3 and -293 to -3$)$ of the PANDER promoter constructs and treated with varying concentrations of glucose. In summary, the minimal element was sufficient to confer glucose-responsiveness of the PANDER promoter (Figure 17B) .

\section{Glucose-Responsive PANDER mRNA Levels in Primary Hepatocytes}

RT-PCR was performed on isolated RNA from primary hepatocytes treated with $0,5.5,11$ or $22 \mathrm{mM}$ glucose to determine relative changes in PANDER levels. This assay revealed a significant 
dose-dependent increase in PANDER message, with the 11 and $22 \mathrm{mM}$ glucose treated groups showing approximately 4 and 9 times the levels of the $0 \mathrm{~mm}$ group, respectively (Figure 17C). Therefore, it appears that the primary hepatocyte mRNA levels of PANDER follow the trend demonstrated by promoter activity in the luciferase assay.

\section{ChREBP Mediated PANDER Expression}

The expression of many glucose-sensitive genes within the liver is determined by the carbohydrate response element binding protein (ChREBP) [119]. ChREBP recognizes conserved carbohydrate response elements (ChoREs) in numerous gene promoters and regulates expression particularly under glucose stimulated conditions. Therefore, this transcription factor was an ideal candidate to investigate potential interaction with the PANDER promoter. To determine the level of ChREBP mediated PANDER promoter activity, initial co-transfection experiments were performed. The PANDER promoter construct was co-transfected with a ChREBP expression plasmid and an Mlx co-activator expression plasmid under increasing glucose conditions and activity was evaluated via luciferase assay. Although modest, PANDER promoter activity was significantly increased in the presence of both ChREBP and Mlx expression plasmids under glucose stimulated and unstimulated conditions (Figure 18A). 
ChREBP Binding to the PANDER Promoter as Determined by Chromatin Immunoprecipitation

To functionally demonstrate that ChREBP is binding directly to the PANDER promoter, we performed chromatin immunoprecipitation. We selected three truncations of the PANDER promoter based upon the potential E-box binding regions located therein: -293/ +25, $-193 /+25$ and -93/+25. Following ChREBP immunoprecipitation of cross-linked primary murine hepatocyte DNA and subsequent PCR amplification with PANDER promoter specific primers, we showed robust amplification at the expected molecular weight (approximately $218 \mathrm{bp}$ ) for the $-193 /+25$ primer pair in the $22 \mathrm{mM}$ condition only with no detected ChIP mediated amplification in the $11 \mathrm{mM}$ condition (Figure 18B). No amplification was observed in the IgG non-specific binding control condition. This region $(-193 /+25)$ contains one full and one partial E-box element and was shown to be the minimal element responsible for baseline promoter activity.

\section{E-box 3 Deletion Impairs Basal and Glucose-Stimulated PANDER Promoter Activity}

Site Directed Mutagenesis was performed to eliminate the third E-box element from the PANDER/luciferase promoter construct. This element was the only full E-box element contained within 
the region identified for ChREBP binding by chromatin immunoprecipitation. Following site-directed mutagenesis and confirmation by sequencing, E-box 3 deleted PANDER luciferase plasmids were transfected into BNLCL2 cells and assayed in comparison to the PANDER/luciferase parental construct (-493/3). Under basal conditions (5.5 mM glucose), the activity of the E-box 3 deletion construct was significantly reduced by approximately $60 \%$ as compared to the parental construct (Figure 19A). Glucose-responsiveness was then assessed in the absence of E-box 3. The overall activity and the glucose response of the Ebox 3 construct was significantly reduced when compared to the 493/-3 construct under all conditions (Figure 19B).

\section{Hepatic PANDER Levels are Increased in Diabetic NOD mice}

To further examine the impact of glucose on hepatic PANDER expression, we evaluated the T1D model NOD mouse. This was chosen to determine if extremely high glucose concentrations as observed in diabetic NOD mice would also stimulate hepatic PANDER expression. PANDER levels were examined from livers obtained from recent onset diabetic and non-diabetic NOD mice by RT-PCR analysis. The average blood glucose levels were approximately 386 and $135 \mathrm{mg} / \mathrm{dL}$ in the diabetic and non-diabetic groups, respectively. PANDER was significantly elevated in the diabetic NOD mice (approximate 7 fold increase) as compared to 
their non-diabetic counterparts demonstrating that increased hepatic PANDER expression may reflect and be directly regulated by increased circulating glucose (Figure 20).

\section{Acknowledgements}

I would like to thank the Moffitt Sequencing Core and especially Dr. Lan Min Zhang for assisting with sequencing. I would also like to thank Dr. Howard C. Towle of the University of Minnesota for providing the ChREBP expression plasmids and Dr. Grace $C$. Dougan for providing the murine liver samples. In addition, I would like to thank Dr. Mark Atkinson for providing the NOD mouse liver samples. I would especially like to thank my mentor, Dr. Burkhardt, for his assistance in study design and data analysis. 
A

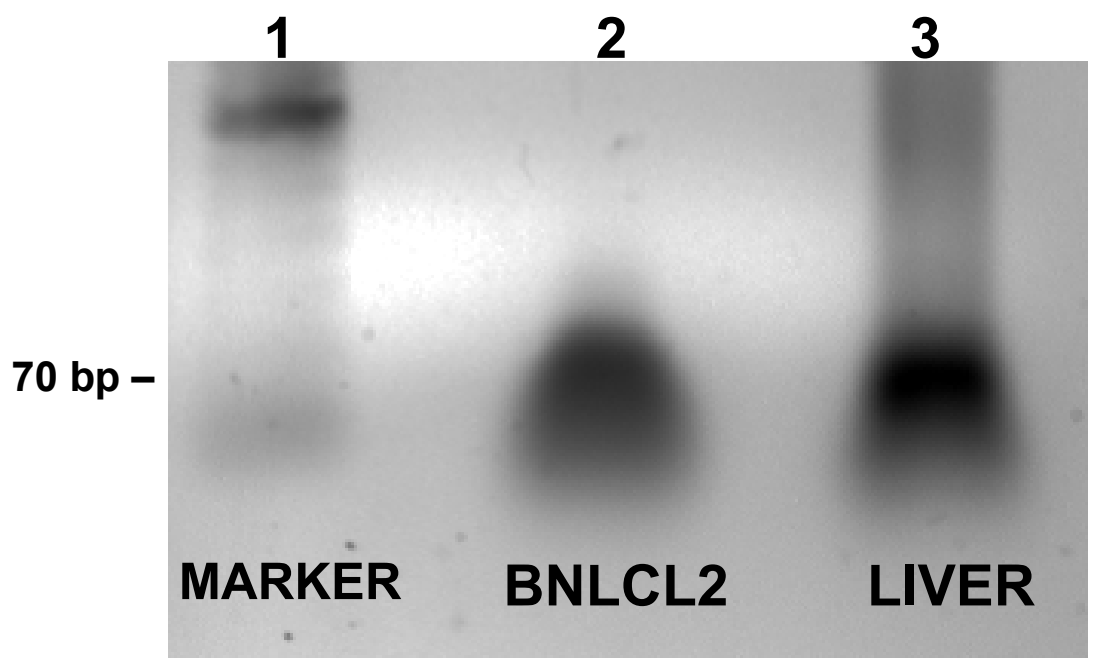

Figure 12: Identification of the murine hepatic transcriptional start site. The hepatic transcriptional start site was determined by 5' RNA ligase mediated rapid amplification of CDNA ends (RLM-RACE) using total RNA from murine embryonic hepatocytes (BNLCL2) and primary murine liver tissue (B6SJLF). (A) RLM-RACE PCR products were analyzed on a 2\% agarose gel. The resultant products after 2 rounds of nested PCR are shown. Lane 1 is the product from BNLCL2 cells and Lane 2 is the product from murine liver lysate. (B) The DNA sequence from -829 to +29 is shown. The identified transcriptional start site is bolded and denoted by +1 hepatic. The previously identified pancreatic start site is denoted by +1 pancreatic. Putative transcription factor binding sites as predicted by MatInspector 7.0 (Genomatix) are shown and underlined. These sites include $A$ and E box elements, hepatocyte nuclear factor (HNF), signal transducer and activator of transcription (STAT) and octamer binding protein-1 (Oct-1). The PANDER translational start codon ATG is italicized and located at the end of the nucleotide sequence. (C) Schematic of the alternative splice variants of the murine PANDER gene. The murine PANDER gene exists as two splice variants, differing by a truncation of the $5^{\prime}$ end. The first spice variant is the gene investigated in this study. 
B

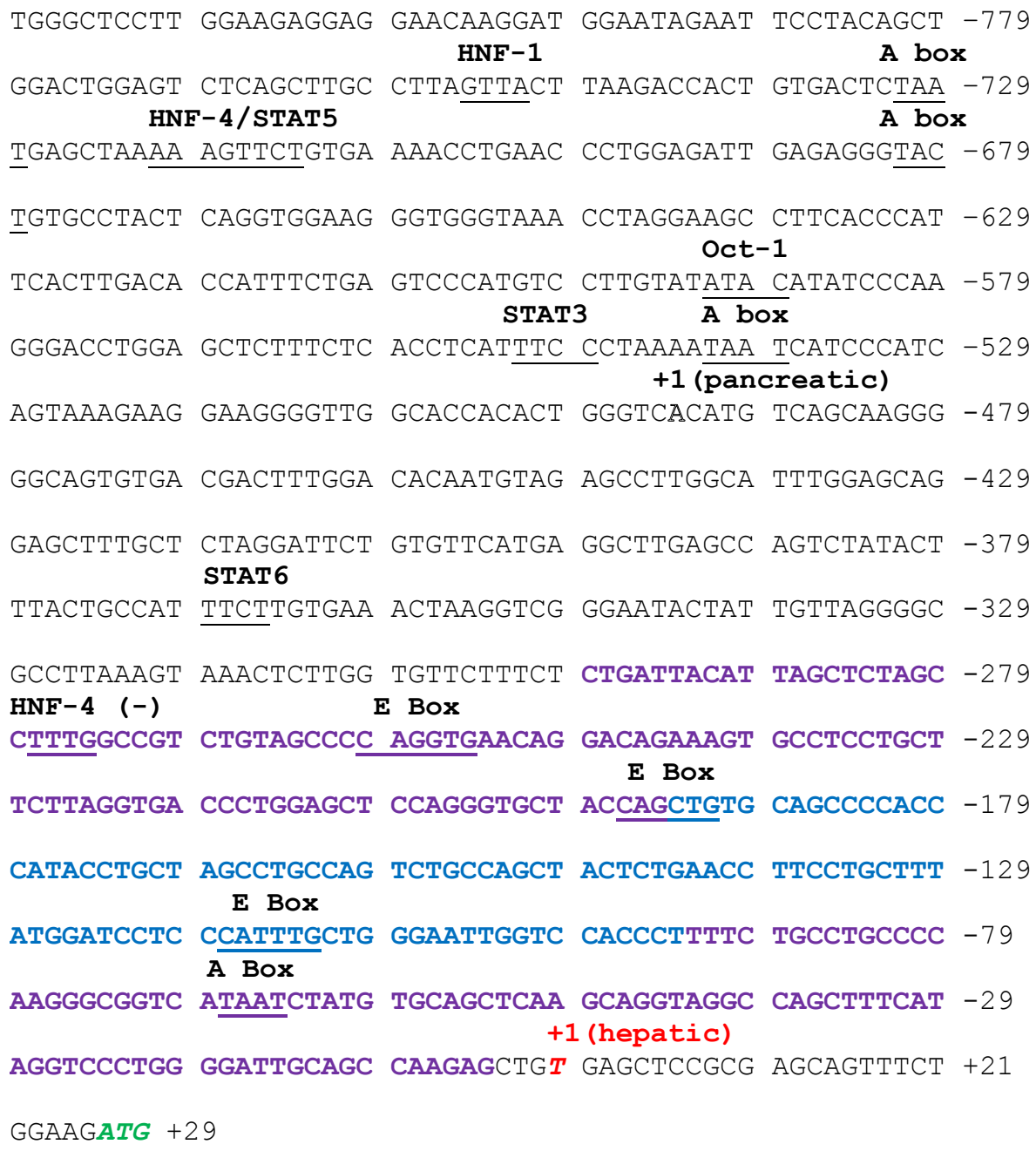

Figure 12: continued. 
C

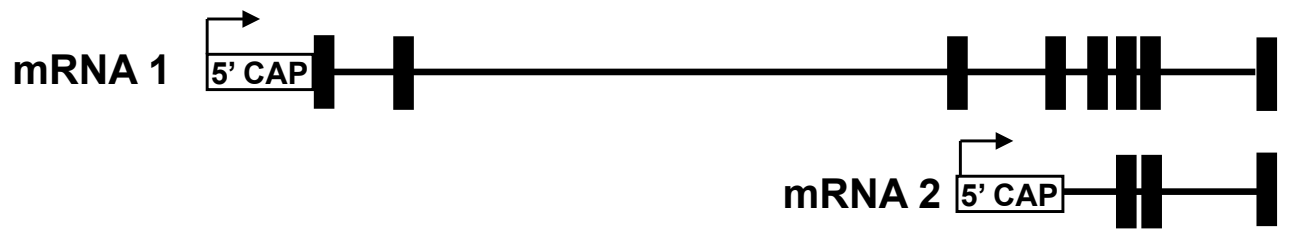

Figure 12: continued. 


\section{A}

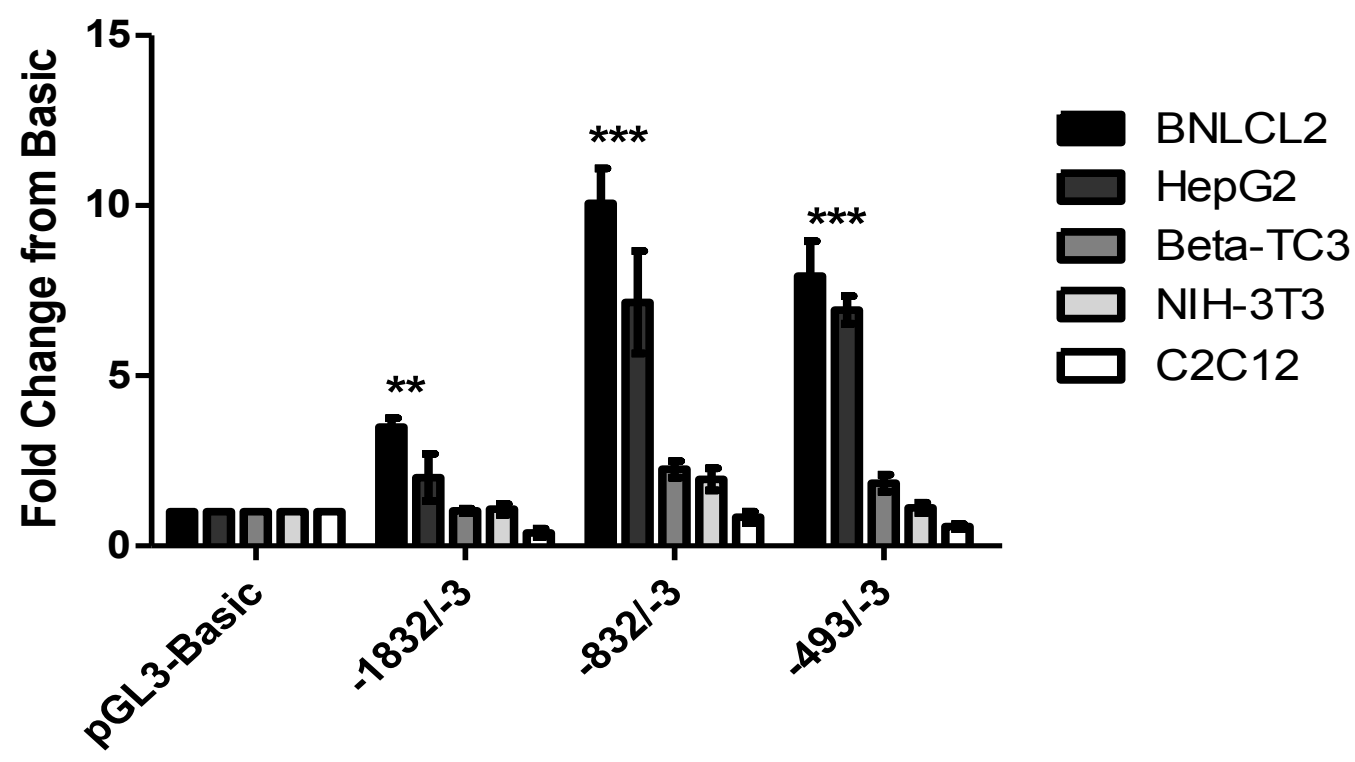

Figure 13: Cell specificity and baseline activity of the murine PANDER promoter. (A) PGL3 luciferase reporter plasmid containing the -832 to -3 region of the PANDER promoter upstream of the luciferase gene was transfected into BNLCL2, HepG2, $\beta$-TC3, NIH3 T3 and C2C12 cells. Luciferase activity was measured following transfection under basal glucose conditions and is reported as a fold change over the promoterless PGL3-basic plasmid. Normalized promoter-less pGL-3 basic plasmid is set at 1 . All data are shown as mean $\pm S . E$. from 3 independent experiments with each PANDER/luciferase construct transfected in triplicate. ** $P<$ $0.01, * \star \star \quad P<0.001$ as compared to the promoterless plasmid as determined by ANOVA. (B) A commercially prepared western blot panel containing several murine tissues (Zyagen) was probed for murine PANDER. The resultant bands are shown for PANDER positive detection. 
B

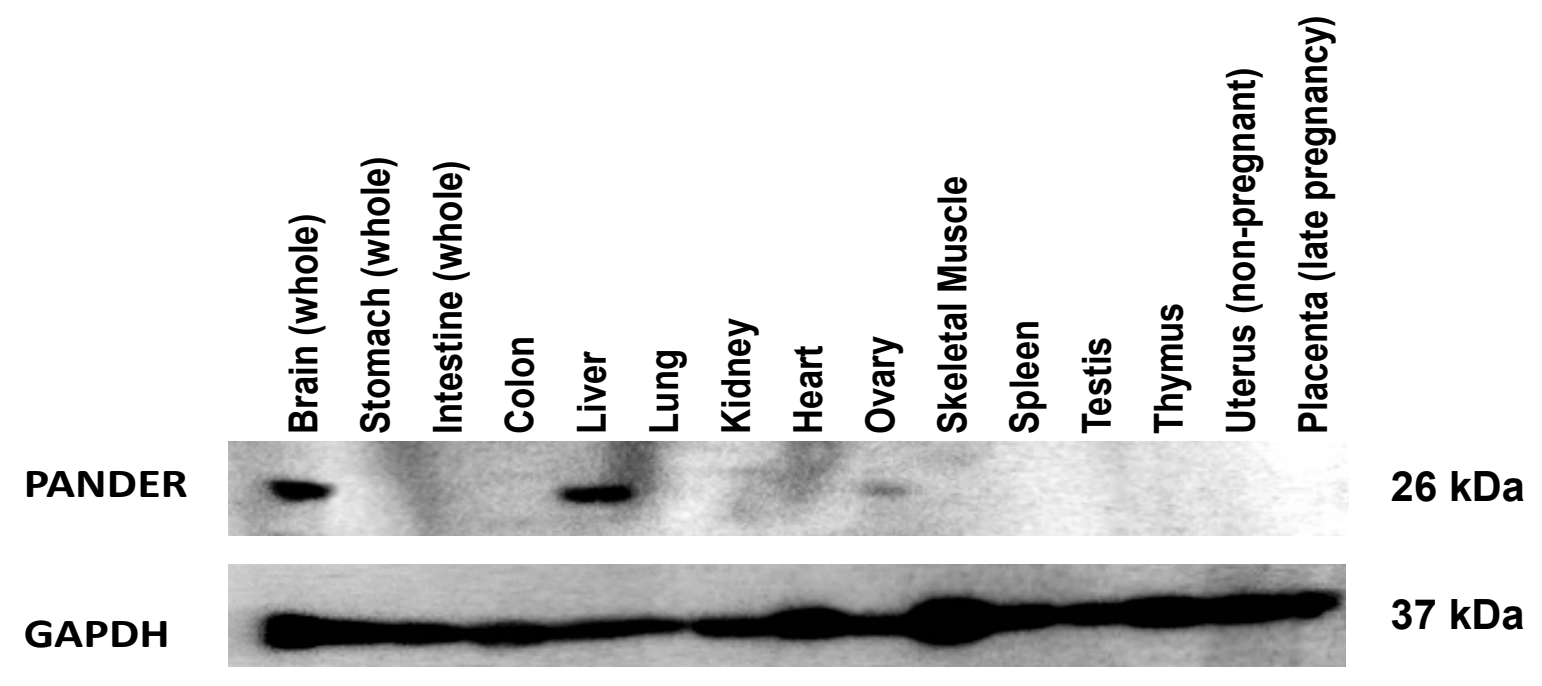

Figure 13: continued. 


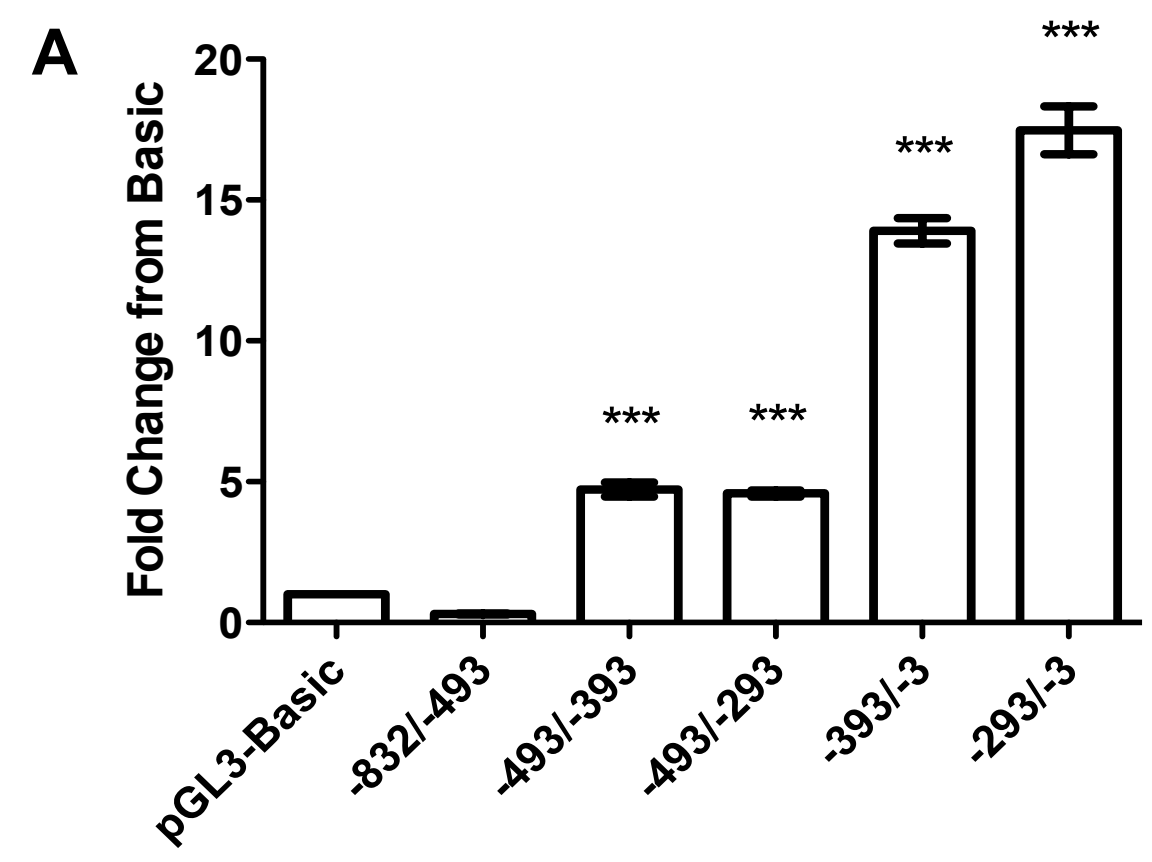

Figure 14: PANDER promoter deletion analysis. (A) PGL-3 luciferase reporter plasmids containing various truncations (as shown) of the PANDER promoter were obtained and transfected into BNLCL2 cells. The resultant luciferase activity is shown. (B) Several pGL-3 luciferase constructs were created with new truncations of the PANDER promoter encompassing the translational start codon. To identify the minimal element necessary for glucose-responsiveness in the PANDER promoter, four luciferase reporter constructs were used containing the $493 /-393,-393 /-293,-393 /-3$ and $-293 /-3$ promoter regions. (A) These constructs were transfected into BNLCL2 cells and treated with $0 \mathrm{mM}, 5.5 \mathrm{mM}$ or $22 \mathrm{mM}$ glucose, as described previously. Luciferase activity was measured after $18 \mathrm{~h}$ and is reported as a fold change from the $0 \mathrm{mM}$ condition. Normalized $0 \mathrm{mM}$ condition is set at 1 . All data are shown as mean $\pm S . E$. from 2 independent experiments with each PANDER/luciferase construct transfected in triplicate. These constructs were transfected into BNLCL2 cells. Resultant luciferase activity is shown. ** and $* * \star$ denotes a $P-$ value $<0.01$ and $<0.001$, respectively, when compared to the

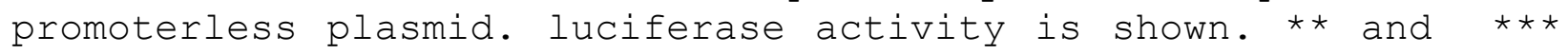
denotes a P-value $<0.01$ and $<0.001$, respectively, when compared to the promoterless plasmid. 


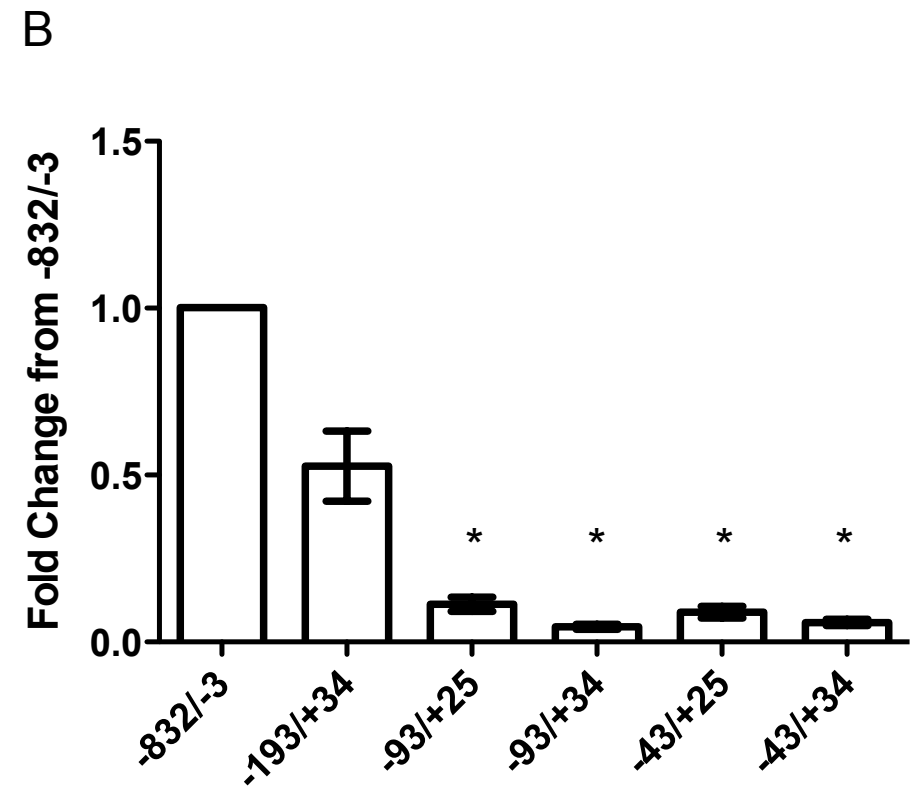

Figure 14: continued. 


\section{A}

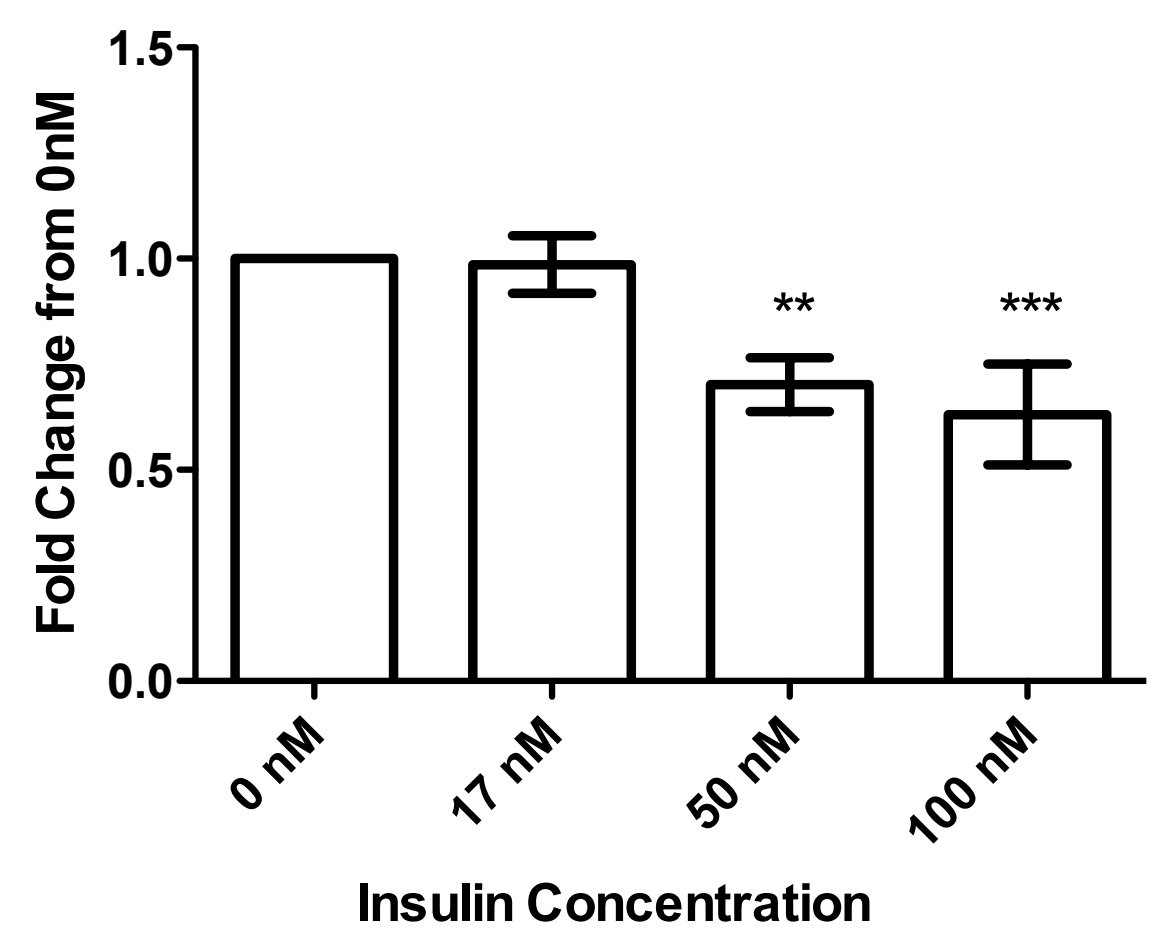

Figure 15: Insulin and palmitate inhibit PANDER promoter activity. The $-832 /-3$ PANDER promoter construct was used to determine the level of stimulation of the promoter in response to several secretagogues. (A) HepG2 cells and (B) BNLCL2 cells were transfected with the PANDER promoter construct. After $2 \mathrm{~h}$ media containing various concentrations of insulin was applied. Luciferase activity was measured after $18 \mathrm{~h}$ and is reported as a fold change from the $0 \mathrm{mM}$ condition. Normalized $0 \mathrm{mM}$ condition is set at 1. All data are shown as mean \pm S.E. from 2 independent experiments with each PANDER/luciferase construct transfected in triplicate. (C) BNLCL2 cells were transfected with the PANDER promoter construct and treated with media containing various concentrations of palmitate alone or palmitate with glucose (D) after $2 \mathrm{~h}$. Luciferase activity after $18 \mathrm{~h}$ is shown as a fold change from the $0 \mathrm{mM}$ condition. The asterisk denotes a p-value of less than 0.05 when compared to the untreated condition. 
B

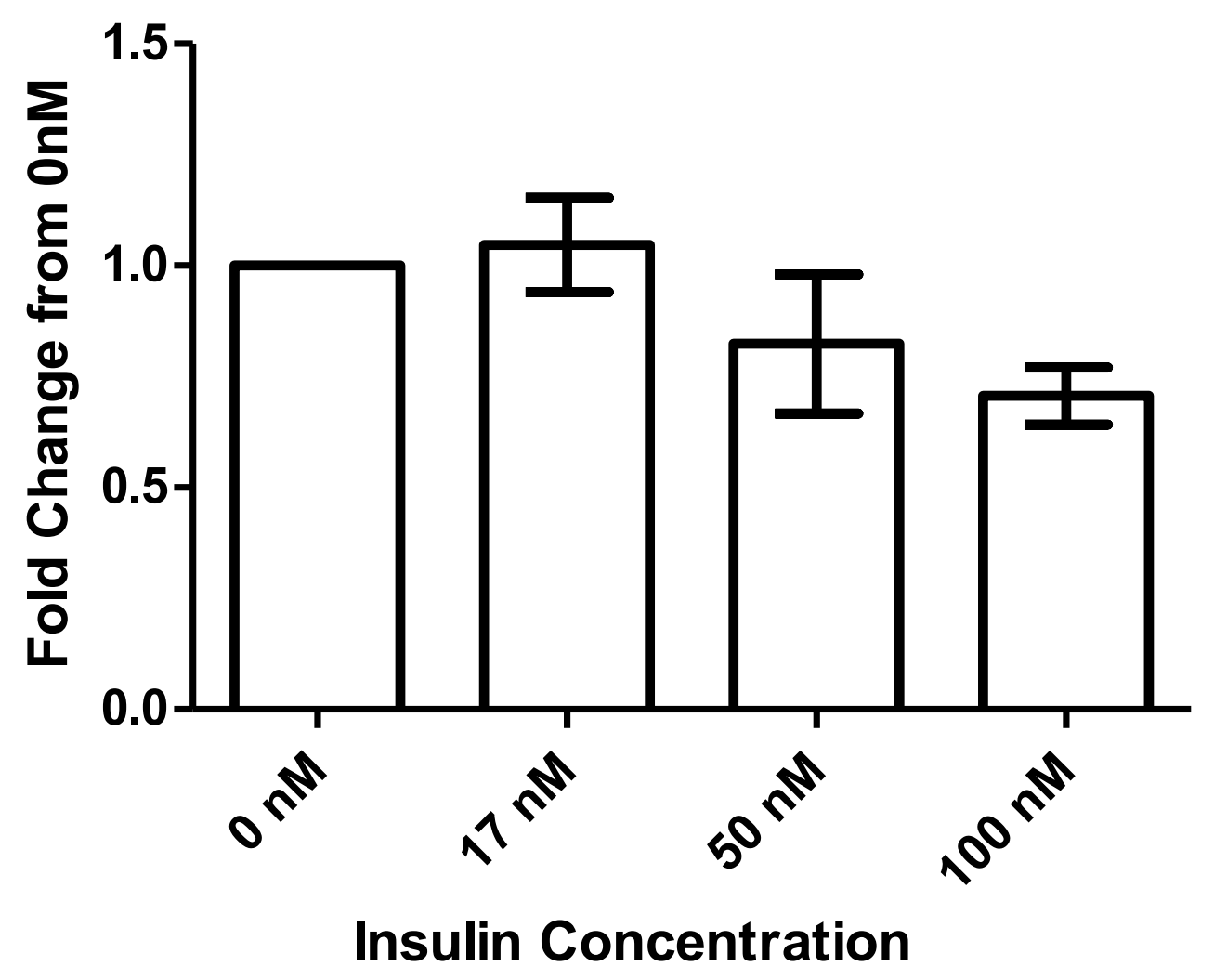

Figure 15: continued. 


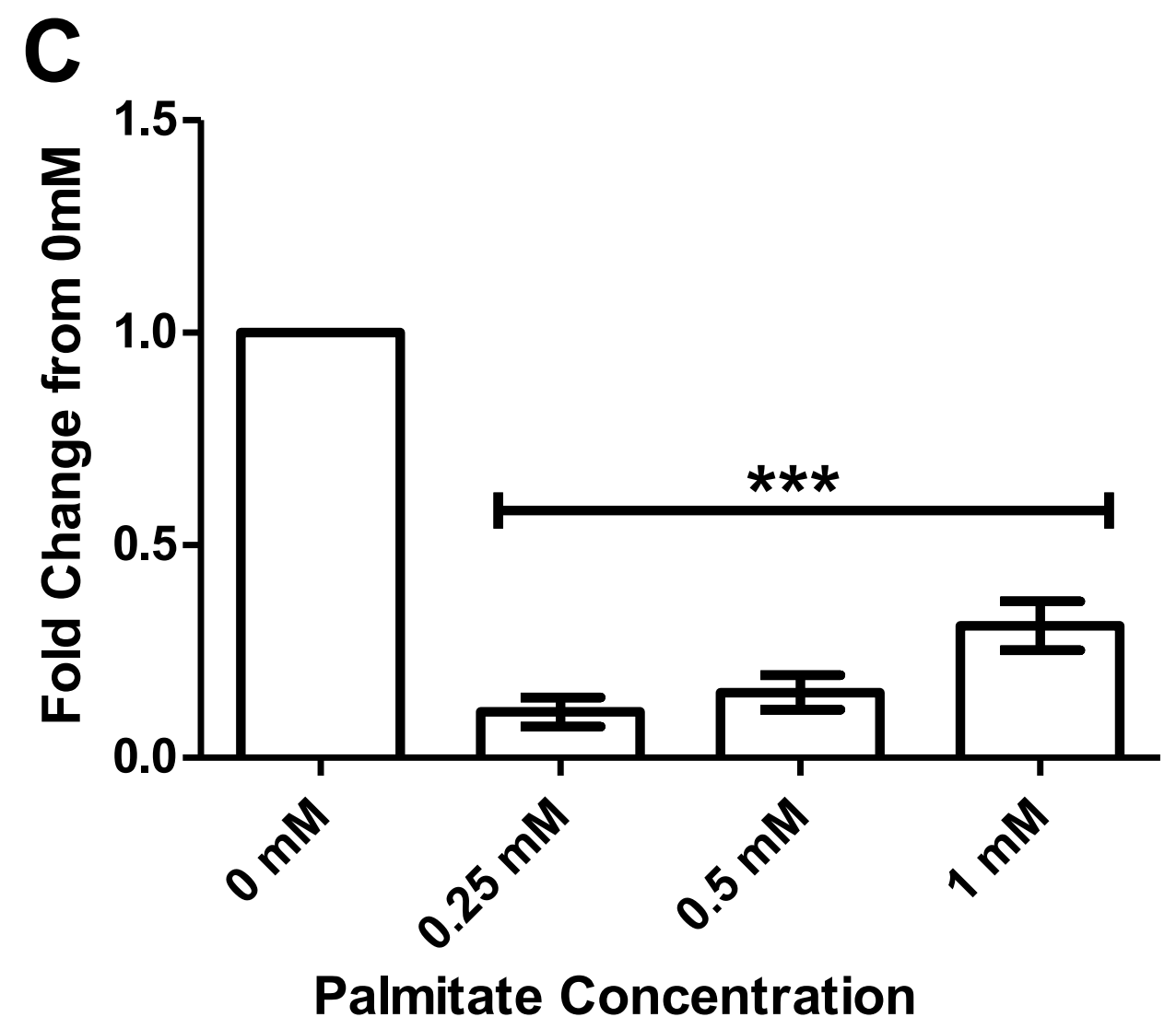

Figure 15: continued. 
D

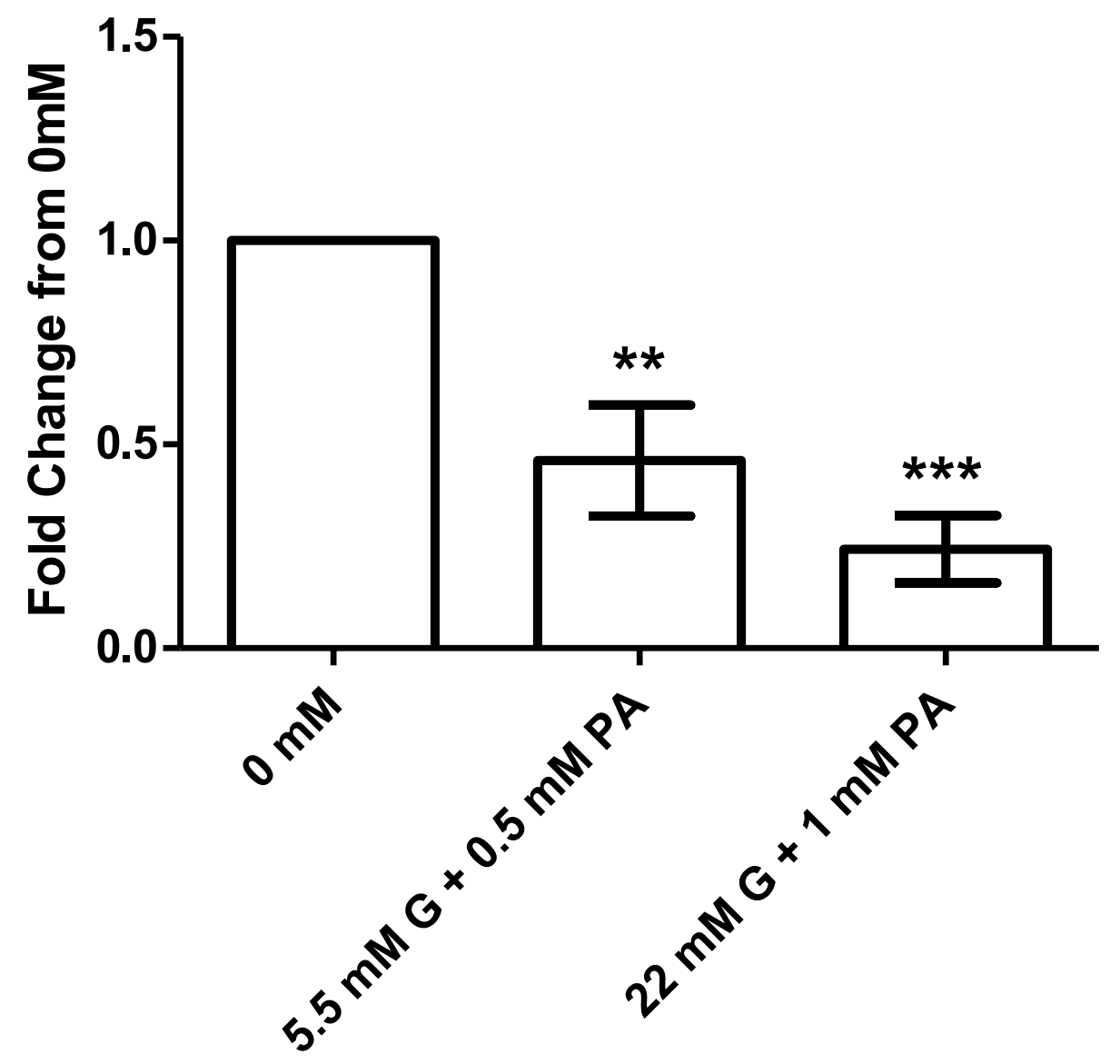

Figure 15: continued. 


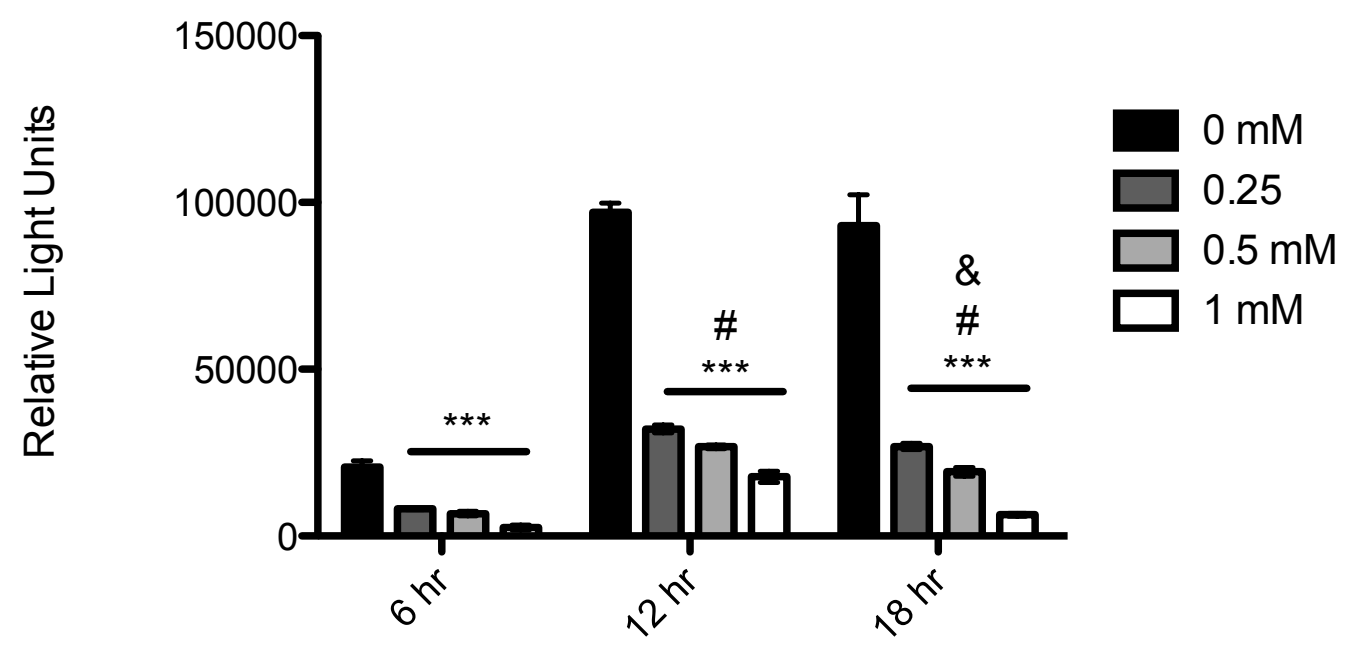

Time Post-Treatement

Figure 16: Palmitate Stimulation of $\beta-T C 3$ cell line. (A) $\beta-T C 3$ cells were treated with $0,0.25,0.5$ and $1 \mathrm{mM}$ palmitate and luciferase assay was performed at 6,12 and 18 hours posttreatment. $* \star \star$ represents a p-value of $<0.001$ compared to the 1 $\mathrm{mM}$ treatment. \# represents a p-value of $<0.05$ between the 0.25 and $1 \mathrm{mM}$ treatment groups. \& represents a p-value of $<0.05$ between 0.25 and $0.5 \mathrm{mM}$ treatment groups. (B) $\beta$-TC3 cells were treated with $0.95 \%$ ethanol or normal culture medium following transfection with a PANDER promoter construct and a luciferase assay was performed. 
B

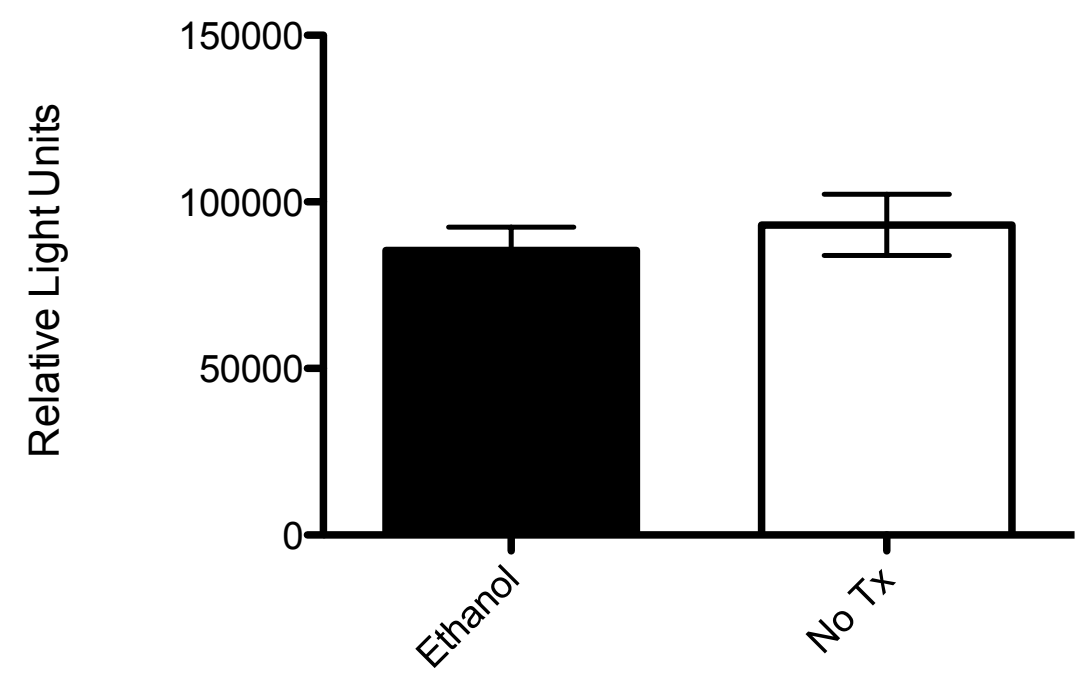

Figure 16: continued. 


\section{A}

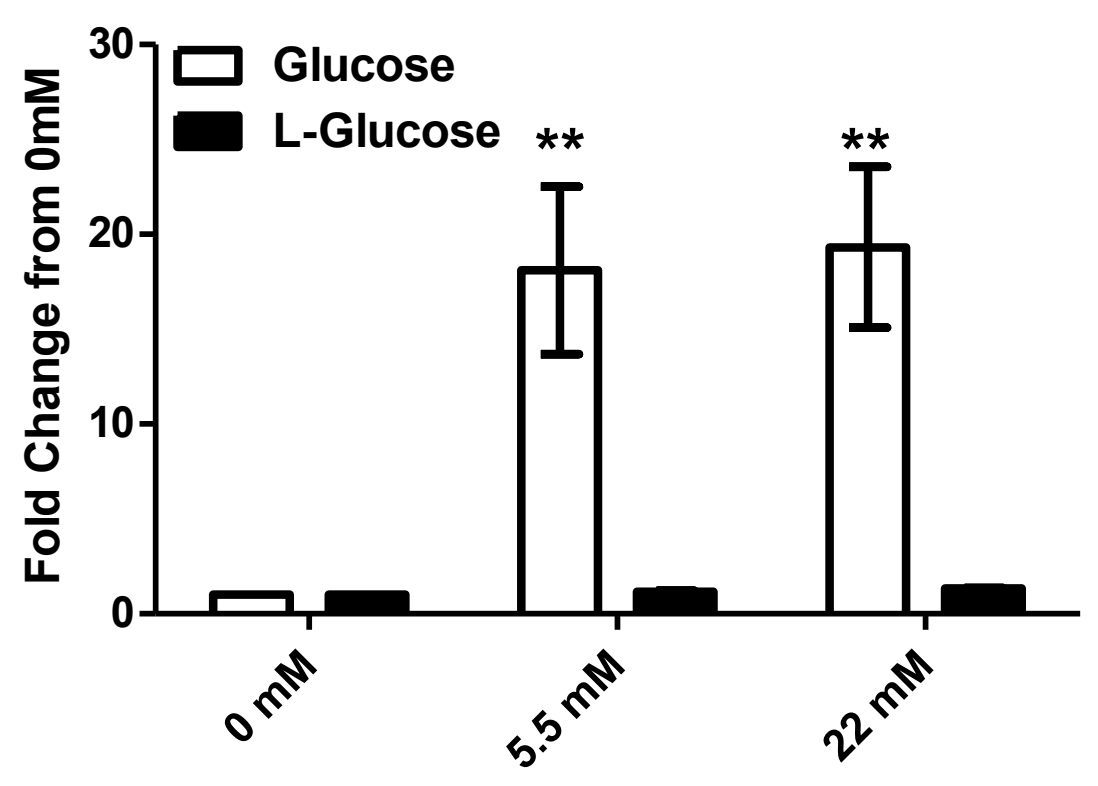

Glucose Concentration

Figure 17: Glucose-responsiveness of the PANDER promoter. Glucose responsiveness of the PANDER promoter was assessed in the $-832 /-3$ construct. (A) This construct was transfected into BNLCL2 cells and treated with $0 \mathrm{mM}, 5.5 \mathrm{mM}$ or $22 \mathrm{mM}$ glucose, as described previously. (B) To identify the minimal element necessary for glucose-responsiveness in the PANDER promoter, two luciferase reporter constructs were used containing the $-393 /-3$ and -293/-3 promoter regions. Luciferase activity was measured after $18 \mathrm{~h}$ and is reported as a fold change from the $0 \mathrm{mM}$ condition. Normalized $0 \mathrm{mM}$ condition is set at 1 . All data are shown as mean $\pm S . E$. from 2 independent experiments with each PANDER/luciferase construct transfected in triplicate. The asterisk denotes a p-value of less than 0.05 when compared to the untreated condition. (C) Relative PANDER mRNA levels were determined in primary hepatocytes treated with $0 \mathrm{mM}, 5.5 \mathrm{mM}, 11$ $\mathrm{mM}$ or $22 \mathrm{mM}$ glucose. Relative $-\Delta \Delta \mathrm{Ct}$ values are reported as a fold change above the $0 \mathrm{mM}$ condition. All data are shown as mean tS.E. from 2 independent experiments. $\star \star \star ~ P<0.001$ from $0 \mathrm{mM}$ glucose condition, \# $\mathrm{P}<0.05$ from $5.5 \mathrm{mM}$ condition and \& $\mathrm{P}<$ 0.05 from $11 \mathrm{mM}$ condition. 


\section{Glucose Concentration}

B

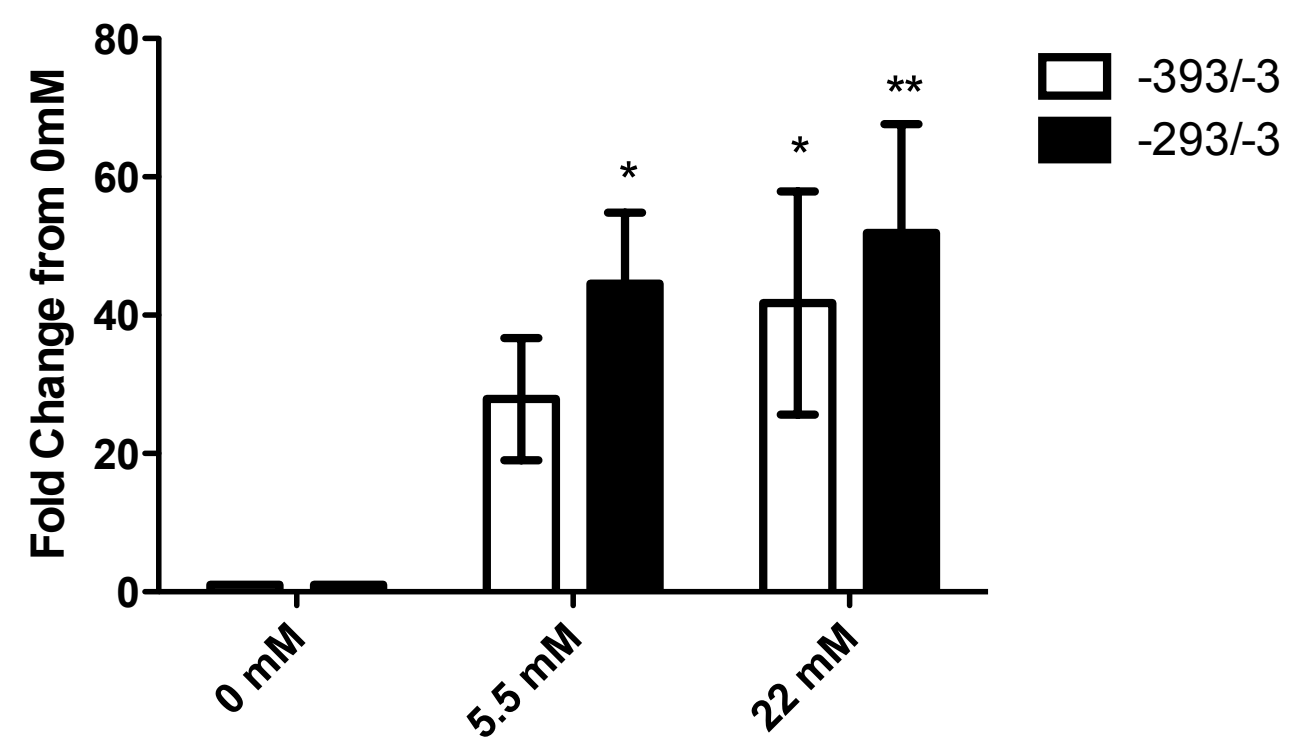

Glucose Concentration

Figure 17: continued. 
C

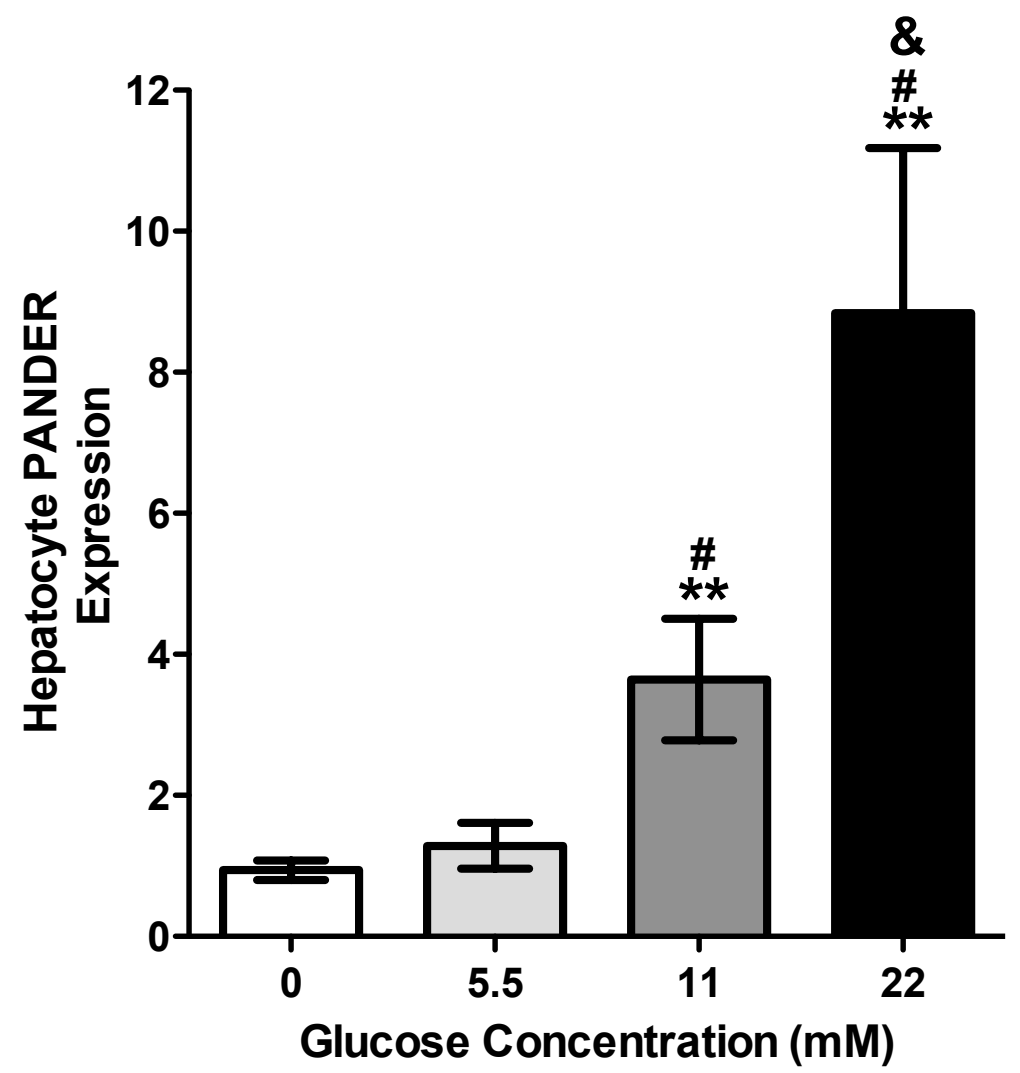

Figure 17: continued. 

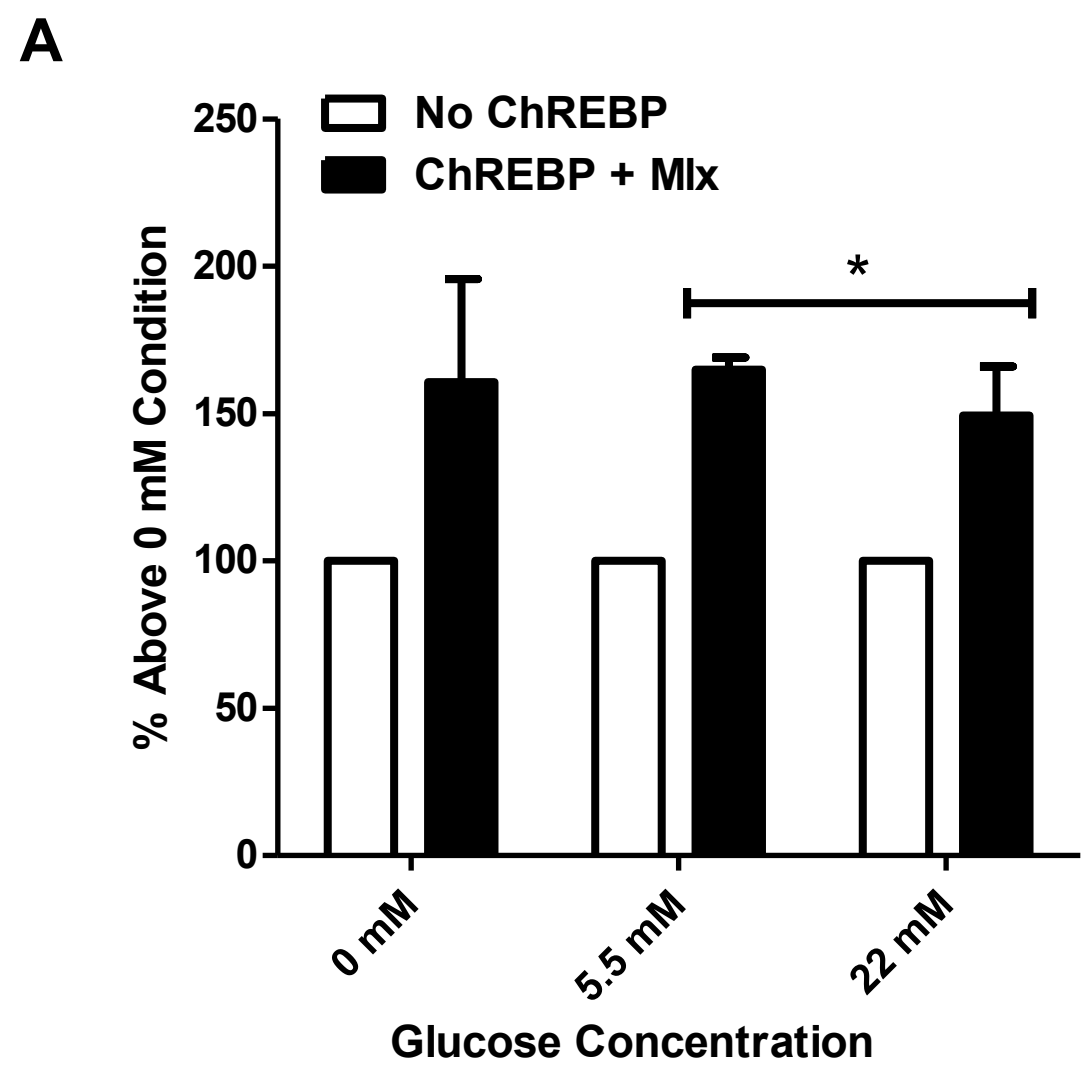

Figure 18: ChREBP mediated activity of the PANDER promoter. (A) Co-transfection experiments were performed to determine the effect of increased carbohydrate responsive element binding protein (ChREBP) on PANDER promoter activity. BNLCL2 cells were transfected with either the $-832 /-3$ PANDER promoter construct alone or in combination with the wild-type ChREBP expression plasmid along with the Mlx coactivator expression plasmid and stimulated with $0 \mathrm{mM}, 5.5 \mathrm{mM}$ or $22 \mathrm{mM}$ glucose. Luciferase activity after $18 \mathrm{~h}$ is reported as fold change over the PANDER promoter All data are shown as mean \pm S.E. only condition. Normalized promoter only condition is set at 1 . from 2 independent experiments with each PANDER/luciferase construct transfected in triplicate. The asterisk denotes a p-value of less than 0.05 when compared to the promoter only condition. (B) Chromatin immunoprecipitation was performed on crosslinked DNA obtained from primary murine hepatocytes treated with $11 \mathrm{mM}$ or $22 \mathrm{mM}$ glucose to determine binding of ChREBP to the PANDER promoter. The representative PCR results using primers specific to the $-193 /+25$ region of the PANDER promoter following ChIP purification on a $1 \%$ agarose gel are shown. 


\section{B}

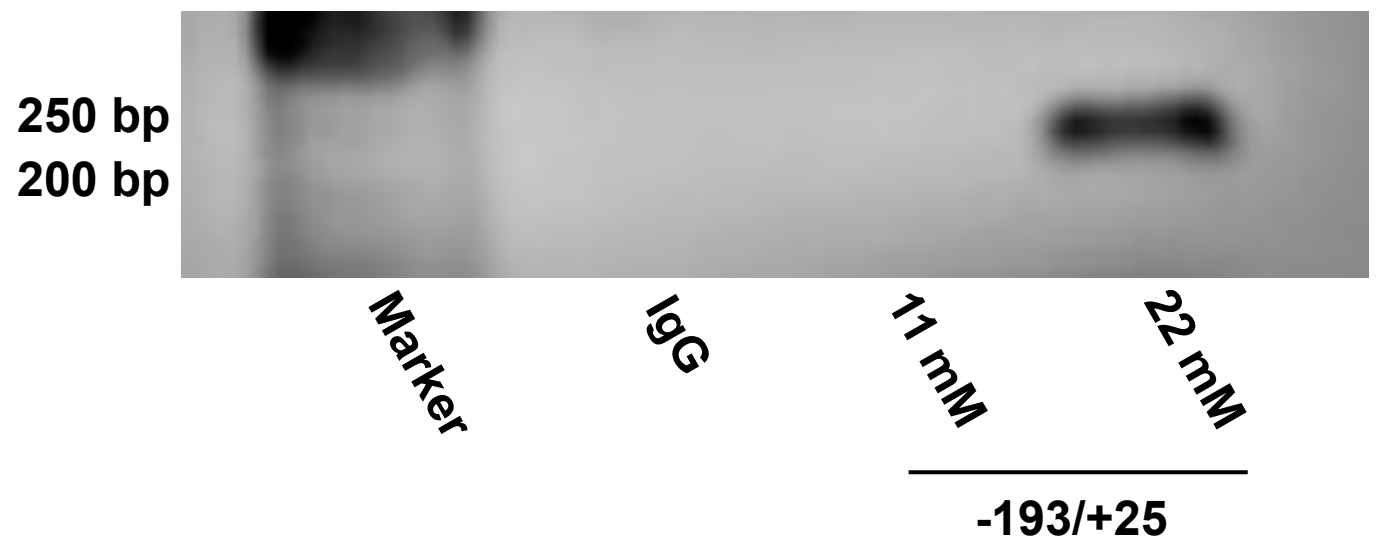

Figure 18: continued. 


\section{A}

Parental Construct (-493/-3):

E-BOX 3
ATGGATCCTC CCATTTGCTG GGAATTGGTC CACCCTTTTC TGCCTGCCCC -79

Mutant construct (E-Box 3 Deletion):

ATGGATCCTC CAATCCTG GGAATGGTC CACCCTTTTC TGCCTGCCCC -79

Figure 19: E-box 3 deletion decreases basal and glucose stimulated PANDER promoter activity. The third E-box element was removed via site-directed mutagenesis from the parental (-493/3) PANDER/luciferase plasmid. (A) Baseline activity of the PANDER promoter under basal cell culture conditions of $5.5 \mathrm{mM}$ glucose was assessed in BNLCL2 cells. Data is presented as percentage of the -493/-3 PANDER/luciferase activity. (B) BNLCL2 cells were treated with $0 \mathrm{mM}, 5.5 \mathrm{mM}, 11 \mathrm{mM}$ or $22 \mathrm{mM}$ glucose after transfection with either the $-493 /-3$ PANDER/luciferase plasmid or the E-box 3 deleted construct. Data is presented as the fold change in relative activity from the $-493 /-3$ PANDER/luciferase plasmid in the $0 \mathrm{mM}$ condition. Luciferase activity was measured after 18 hours. All data are shown as mean $\pm S . E$. from 2 independent experiments with each transfection performed in triplicate. $\star * \star ~ P<0.001$ from $0 \mathrm{mM}$ condition and \# $\mathrm{P}<0.05$ from $5.5 \mathrm{mM}$ condition. 
B

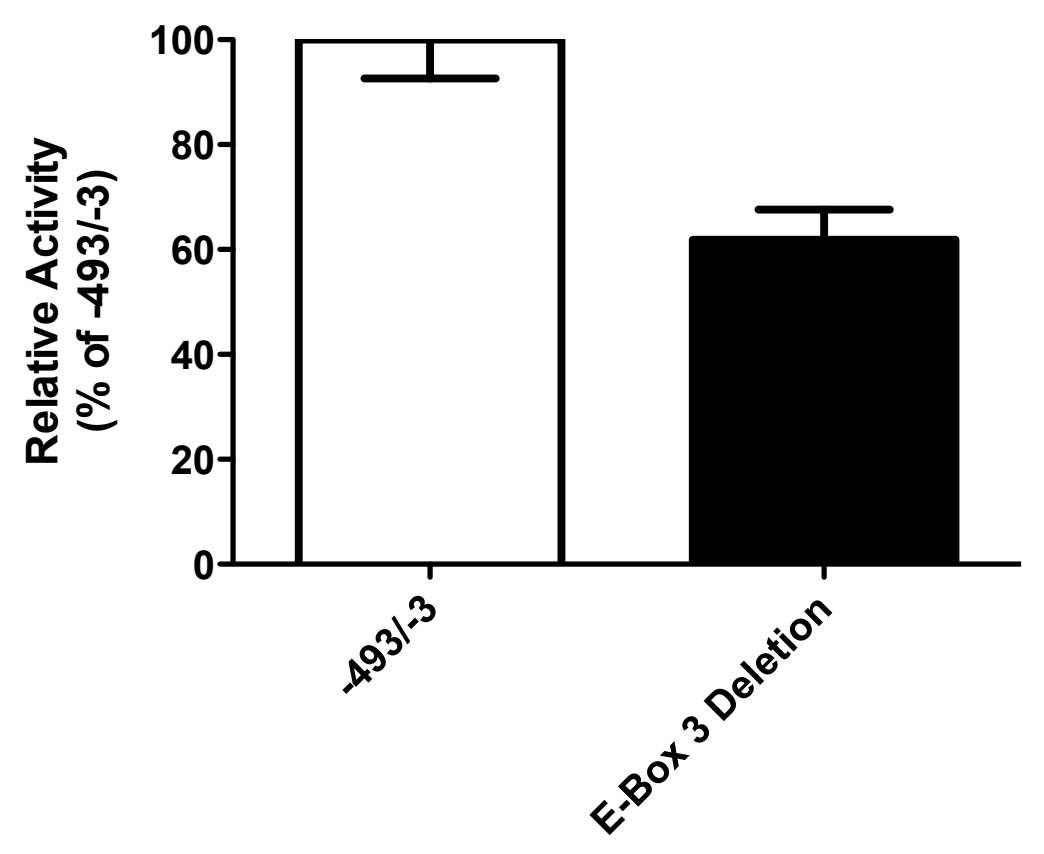

Figure 19: continued. 
C

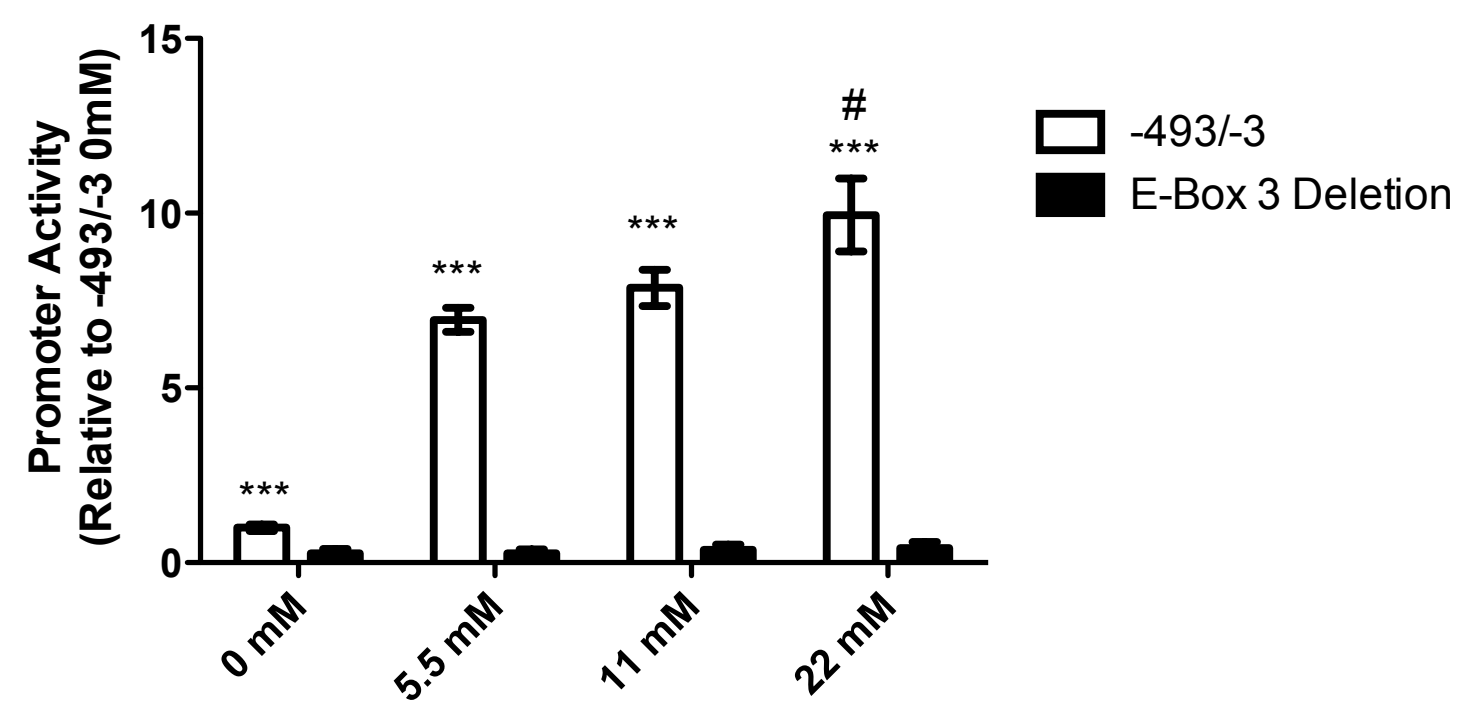

Figure 19: continued. 


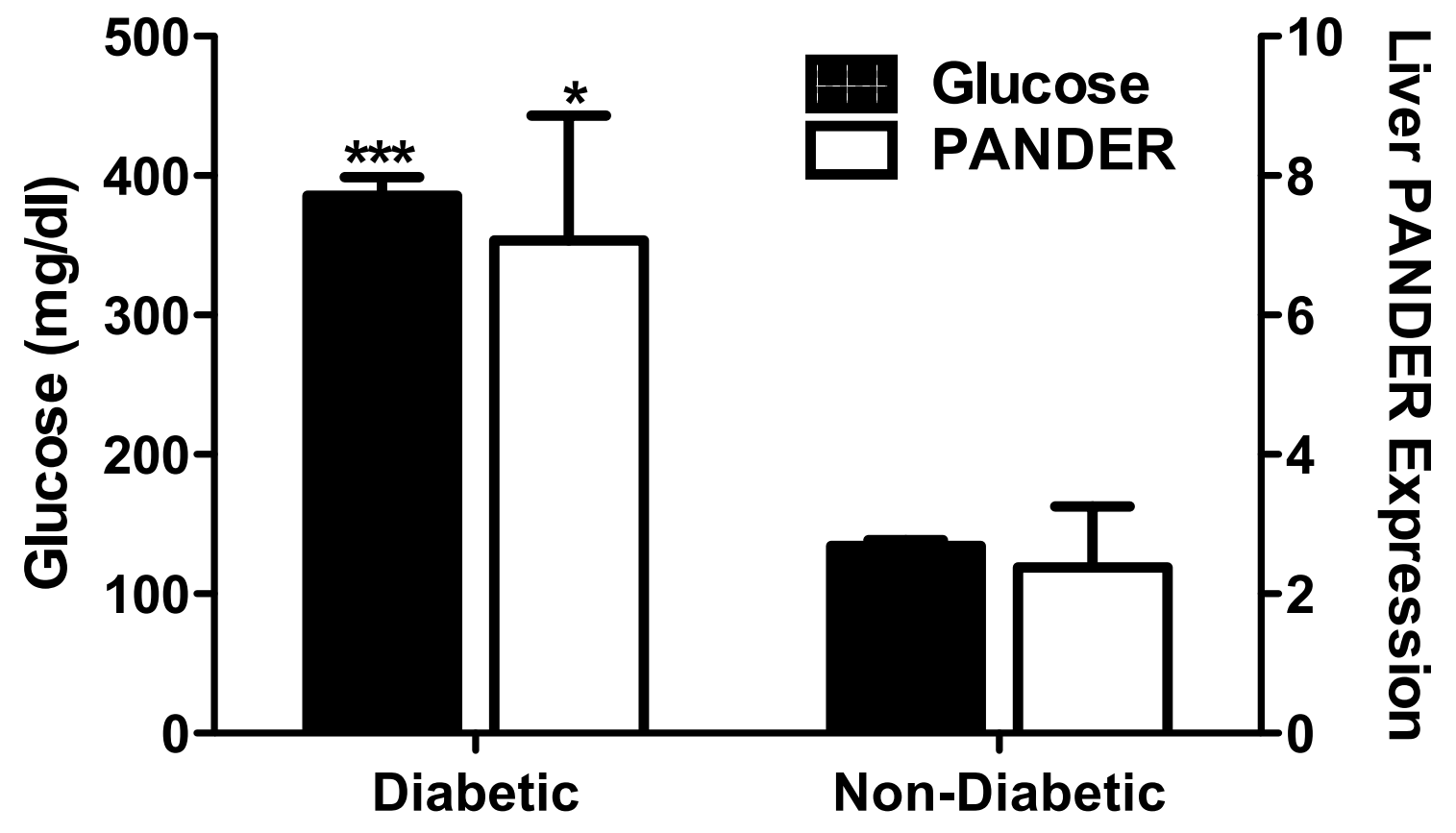

Figure 20: Hepatic PANDER levels in diabetic and non-diabetic NOD mice. RT-PCR was performed on livers obtained from 28-weekold female NOD mice. Glucose concentration (left y-axis) and relative PANDER levels (right y-axis) are presented for diabetic and non-diabetic NOD mice. RT-PCR PANDER expression levels were normalized to non-diabetic NOD mice. All data are shown as mean \pm S.E. $* \star \star \quad P<0.001$ and $\star P<0.05$. 


\section{CHAPTER 4: DISCUSSION}

\section{Note to Reader}

Portions of this chapter have been previously published in Molecular and Cellular Endocrinology, 2015, 413: 101-112, and have been reproduced with permission from Elsevier. Whitney A. Ratliff designed the study, acquired, analyzed and interpreted the data and wrote the manuscript. Mark G. Athanason, Alicia C. Chechele, Melanie N. Kuehl, Amanda M. Fernandez and Catherine B. Marelia assisted with data acquisition. Brant $R$. Burkhardt designed the study, interpreted the data and contributed to manuscript writing. All authors read and approved the final manuscript.

This study provides the first in-depth analysis of the activity and regulation of the PANDER promoter specifically within the liver. Our findings have demonstrated that PANDER protein is robustly expressed in liver tissue and this is driven by the cell type specific activity of the PANDER promoter with glucose being the most potent secretagogue. Taken together, our data is highly supportive of prior in-vivo studies examining the biological function of PANDER in various murine models. For 
example, Li et al. demonstrated that PANDER mRNA and protein levels were increased in the livers of $d b / d b$ mice as compared with $d b / m$ mice. Their findings also showed that PANDER protein expression was increased in the livers of mice fed a high fat diet for 12 weeks. Physical exercise attenuated hepatic PANDER expression. Similarly, increased hepatic PANDER expression in the livers of HFD-fed rats was also reversed by physical exercise, concomitant with an amelioration of fatty liver [67]. In addition, our findings support the recent analysis of the PANDER transgenic model whereby hepatic PANDER protein levels were found to be increased in the post-prandial state in both wild-type and PANTG mice [70]. Our studies suggest that the increase in PANDER expression may be more due to the hyperglycemic state of the previously mentioned models more so than increased systemic lipidemia or insulinemia.

In addition, our reported findings are in strong concordance to that observed with PANDER expression in the endocrine pancreas. Previous studies have shown that the PANDER promoter is highly glucose-responsive within the pancreas and that this is due, in part, to regulation by the pancreas-specific transcription factor Pancreatic Duodenal Homeobox-1 (PDX-1) [111, 118]. Our characterization of a novel liver-specific transcriptional start site suggests that transcriptional regulation of the PANDER 
promoter within the liver is under a different TF-mediated mechanism as that found for expression from the endocrine pancreas, despite both being glucose-responsive. There are many genes that are regulated by multiple transcriptional start sites. In fact, it is estimated that approximately 35\% of genes annotated by GENCODE possess more than one start site [120]. Data suggest that these start sites are regulated differently depending on their position relative to the gene. One study found that transcription start sites that are located far downstream are more responsive to transcription factors, whereas upstream start sites may be regulated more by chromatin organization and other factors [121]. Another major determinant of alternative transcription start sites is with regard to cell type specificity. Caninci et al. described that mammalian promoters can be separated into two classes: (1) conserved TATA box-enriched promoters that contain a defined site of TSS and (2) more plastic, broad and evolvable CpG-rich promoters [122]. Their quantitative analysis of promoter usage in different tissues demonstrated that differentially regulated alternative TSSs are a common feature in protein-coding genes. Other investigators have also demonstrated via large scale TSS analysis that promoters do not always initiate transcription at a single defined location of base pairs but rather at multiple start sites located across a broad region [123]. The PANDER 
promoter does lack a TATA box and therefore may fall into the category of promoters that may contain alternative TSS that are regulated by different mechanisms depending on the tissue and secretogues of activation.

ChREBP serves a critical role in hepatic de novo lipogenesis and potentially the onset of T2D and metabolic syndrome [124]. ChREBP appears to regulate approximately 50\% of the genes critical for hepatic lipogenesis [125]. Potentially acting in concert with PANDER, ChREBP may be synergistically both promoting and enhancing the PANDER induced hepatic lipogenesis observed in PANDER overexpressing animal models [67, 70]. Our recent characterization of our PANTG murine model revealed that PANDER does globally activate hepatic lipid metabolism. A comprehensive proteomic analysis of livers obtained from PANTG mice utilizing stable isotope labeling of amino acids in cell culture (SILAC) was performed. This quantitative proteomic strategy metabolically labels the entire proteome and allows for identification and network elucidation by mass spectrometry analysis followed computational determination of differentially expressed proteins and networks. Of the approximately 1600 proteins identified and quantitated, the differentially expressed proteins found in the PANTG revealed that lipid metabolism was the top associated network that was significantly 
increased. Computational analysis predicted an increased quantity of triacylglycerol. Taken together, we find that both ChREBP and PANDER may be working together to comprehensively impact hepatic lipogenesis. In addition, ChREBP knockout phenotypes may potentially be attributed to the absence of PANDER as well.

In addition, ChREBP may be directly involved with the onset or progression of type 2 diabetes in a manner similar to that reported for PANDER [95, 105, 126]. Expression of ChREBP is increased in the liver of adolescents with glucose intolerance or T2D [127]. Furthermore, hepatic ChREBP levels are increased in obese as compared to lean subjects [128]. In a consistent manner to ChREBP, during pathological conditions (ie. Hyperglycemia or insulinemia), several review articles have detailed that PANDER may impact both T2D and nonalcoholic fatty liver disease by inducing selective hepatic insulin resistance [107, 129]. A single report has revealed that PANDER circulating levels are increased in subjects with T2D [130]. Overexpression of PANDER (systemically or liver-specific) results in a discuption of insulin signaling with observed decreased phosphorylation of protein kinase $B$ (PKB/Akt) resulting in hepatic insulin resistance [70]. Concordantly, the absence of PANDER as observed in multiple PANDER knockout models, results 
in enhanced hepatic insulin sensitivity [71, 77]. In a similar fashion, knockdown expression of ChREBP in ob/ob mice improves glucose tolerance and fatty liver [131]. Therefore, the compilation of data surrounding both PANDER and ChREBP is very consistent in terms of both serving a role in potentially impacting the onset or progression of T2D.

As mentioned previously, the transcriptional regulation of PANDER in the liver and endocrine pancreas may have distinct mechanisms but there may also be some degree of potential overlap with both tissues in regard to ChREBP. ChREBP is also highly expressed in pancreatic islets and appears to be required for glucose-stimulated pancreatic $\beta$-cell proliferation [132]. Pancreatic islets obtained from PANDER knockout mice exhibit impaired glucose stimulated insulin secretion and calcium signaling. Neither islet architecture nor insulin content is affected. Although not precisely examined in this study, ChREBP may still be interacting with the PANDER promoter within pancreatic $\beta$-cells and also serve as both a facilitator and regulator of PANDER function in this cell type. However, ChREBP over-expression in either MIN6 $\beta$-cells or pancreatic islets decreased PDX-1 gene expression at high glucose concentrations [133]. At low glucose, ChREBP inactivation increases PDX-1 expression. Prior studies regarding the PANDER promoter examined 
within pancreatic $\beta-c e l l$ lines revealed that PDX-1 interacts with the various A-box elements within this genomic region and facilitates glucose responsive gene expression [118]. Therefore, based on this evidence, we would speculate that ChREBP promotes PANDER expression in pancreatic $\beta$-cells. Nonetheless, future studies will have to be performed to accurately determine how ChREBP mediates PANDER expression in the endocrine pancreas.

Much of the research surrounding the investigation of PANDER has been associated with T2D [107, 129, 130]. Little has been done determining whether PANDER's impact or hepatic expression may be altered by type 1 diabetes (T1D). This presented study has initially indicated that glucose serves as a potent secretagogue of hepatic expressed PANDER. The NOD model spontaneously develops T1D and will display higher glucose levels in progressively decreasing insulin concentrations due to autoimmune mediated pancreatic $\beta$-cell death. We revealed that diabetic NOD mice display increased hepatic PANDER as compared to non-diabetic matched NOD controls. This indicates that hepatic PANDER expression may be coupled with decreased glycemic regulation as determined by hyperglycemic conditions or reflective of increased pancreatic and systemic inflammation as observed in T1D. Xu et al. demonstrated that Th1 cytokines such 
as IFN-Y are potent stimulators of PANDER expression from pancreatic cell lines and islets [81]. Concordantly, Th1 cytokines have been demonstrated to accelerate T1D autoimmunity [134, 135]. Therefore, increased circulating glucose and cytokine levels as found with TlD conditions may be promoting hepatic PANDER expression and further exacerbating hyperglycemia by also promoting hepatic insulin resistance. Numerous animal models have indicated that PANDER can promote hepatic insulin resistance and lipogenesis [67, 70]. However, further studies are needed to fully delineate glucose from cytokine mediated hepatic PANDER expression but nonetheless provide some evidence that PANDER may be affecting the onset and progression of both T1D and T2D.

Elucidating the mechanism of PANDER regulation in several tissues may potentially provide further insight into understanding the pathology of glucose intolerance, NAFLD and T2D. Our investigation of the PANDER promoter in the context of expression within the liver has revealed: (1) the critical genetic elements of transcriptional regulation, secretagogues mediating expression, and (3) transcription factor regulating glucose responsive expression (ChREBP). In combination, this information can provide essential insight into 
the highly complex molecular mechanisms within hepatic metabolic regulation [136]. 


\section{CHAPTER 5: PANDER INDUCED TRANSCRIPTION FACTOR PROFILING}

\section{Introduction}

While previous aims have focused primarily on the transcriptional regulation of PANDER within the liver, our next aim was to investigate changes in transcription factor networks within hepatocytes in response to treatment with the same secreted PANDER from which the crystal structure was elucidated by AstraZeneca [64]. A transcription factor array was utilized to evaluate levels of 345 transcription factors in untreated and PANDER treated primary hepatocytes. Such a study has never been performed and will provide invaluable information regarding how PANDER may be influencing the transcriptional regulation of downstream genes within the liver. PANDER has been studied almost exclusively with respect to its function as a modulator of metabolic signaling; this study will provide a foundation for the investigation of PANDER within a multitude of pathways and disease states.

\section{Methods}

Primary hepatocyte culture and treatment. To evaluate the response of transcription factor networks in the presence of 
elevated levels of PANDER protein, primary murine hepatocytes (Triangle Research Labs, Triangle Park, NC) were plated on a 6well dish at a concentration of $1.2 \times 10^{6}$ cells/well using the Cryopreserved Animal Hepatocyte Thawing and Plating Medium provided. The following day, media was removed and replaced with fresh media containing no treatment or 10 nM PANDER (AstraZeneca, Södartälje, Sweden). PANDER protein obtained from AstraZeneca was the same isolated secreted PANDER protein used in determining the PANDER crystal structure [64]. After $18 \mathrm{~h}$, cells were removed from culture for nuclear extraction using the Panomics Nuclear Extraction Kit (Panomics, Fremont, CA) according to manufacturer's instructions. One full 6-well plate was harvested per treatment group and combined. Following extraction, protein concentrations were determined using the Pierce 660 nm Protein Assay Reagent (Pierce, Rockford, IL).

Transcription factor protein/DNA Array. To assess the transcription factor response to treatment with PANDER or insulin, the Panomics Protein/DNA Combo Array was used (Affymetrix, Santa Clara, CA). An overview of the principals of this assay is included in Figure 21. Nuclear extracts are mixed with biotinylated oligonucleotide probes, resulting in DNA/protein complexes for those transcription factors present within the nucleus at the time of extraction. A column-based 
system is used to remove unbound probe and elute the DNA/protein complexes. The samples are then denatured to liberate the free probe. These probes are then hybridized to membranes spotted with the consensus binding sequences of 345 transcription factors (map shown in Figure 22). After the hybridization step, the membranes were developed using ECL Western Blotting Substrate (Pierce, Rockford, IL). The membranes were visualized using the Amersham Imager 600 (General Electric Healthcare Life Sciences, Pittsburgh, PA).

Data analysis for TF Array. Relative densitometry analysis was performed on each blot using the ImageQuant TL Version 8.1 analysis software (General Electric Healthcare Life Sciences, Pittsburgh, PA). The identified differentially regulated proteins were assigned fold change values from the untreated condition, in cases where the untreated condition's densitometry value was 0 , the lowest detected densitometry value was assigned. The data was then analyzed using the Ingenuity Pathway Analysis (IPA) program to evaluate canonical pathways.

Western analysis of PANDER in cell lines. This western blot was prepared in collaboration with Punashi Dutta of Dr. Meera Nanjundan's laboratory, according to the Nanjundan lab's protocols using antibody provided by our lab. Protein was 
harvested from several cancerous and noncancerous cell lines; cells were lysed for 1 hour in the appropriate volume of lysis buffer containing $150 \mathrm{mM} \mathrm{NaCl}, 1 \mathrm{mM} \mathrm{MgCl2,} 1 \mathrm{mM}$ EGTA, $50 \mathrm{mM}$ HEPES, 1\% Triton X-100, 10\% glycerol and protease inhibitor tablet (Roche, Madison, WI) in Nanopure water. Cells were collected and centrifuged at 14,000 rpm for 10 minutes at $4^{\circ} \mathrm{C}$. The Bicinchoninic Assay (BCA) Kit (Pierce, Pittsburgh, PA) was used to assess protein concentration of the supernatant. Protein concentration was normalized to the lowest concentrated sample and diluted in lysis buffer. To prepare the samples for loading, $10 \mathrm{ml} 6 \mathrm{X}$ SDS dye was added to $50 \mathrm{ml}$ sample and $20 \mathrm{ml}$ was loaded into each well on a 12\% SDS-PAGE gel. The gel was run for 2 hours at 100 Volts and then transferred to a PVDF membrane using a semi-dry transfer apparatus (Bio-Rad, Hercules, CA) at 0.11 Amps (or 0.15 Amps for 2 gels) for 2 hours. The membranes were washed for 10 minutes in TBST (Bio-Rad, Hercules, CA) on an orbital shaker (Thermo Scientific, Hudson, New Hampshire). Membranes were then blocked for 1 hour in 5\% milk in TBST. Next, the blots were rinsed in TBST and probed for PANDER using the FAM3B M-80 rabbit polyclonal antibody (Santa Cruz Biotechnology, Santa Cruz, CA) at a 1:1000 dilution in commercial StartBlock ${ }^{\mathrm{TM}}$ blocking buffer (Thermo Scientific, Waltham, MA) and incubated overnight on an orbital shaker at $4{ }^{\circ} \mathrm{C}$. The following day, the membranes were washed 4 times for 15 mins each in TBST. 
Following the wash a secondary antibody was applied (goat antirabbit (\#107-5046, Immun-Star, Biorad, Hercules, CA) at a 1:10,000 dilution. After the 1 hour secondary antibody incubation, the blots were washed 6 times for 15 mins in TBST. The blots were developed using enhanced chemiluminescence solution (Immun-Star, Bio-Rad) followed by exposure of the membrane to X-ray film [137]. Densitometry analysis was performed on the ovarian cell lines using ImageJ software (National Institutes of Health, Bethesda, MD).

\section{Results}

Identification of differentially expressed transcription factors in response to PANDER treatment. Densitometry analysis was performed on the untreated and PANDER treated transcription factor array membranes (Figure 23). In total, 63 differentially regulated transcription factors were identified; 55 were upregulated in the PANDER treated condition when compared to untreated and 8 were downregulated. A complete list of the identified factors and their fold change from the untreated condition is shown in Table 2. Of the proteins that were contained within the Uniprot database, the top 10 upregulated were as follows: GATA-2 [138], CEBPA [139], TCF3 [140, 141], PAX6 [142], SP1 [143], YBX1 [144], SAA1 [145], YY1 [146], PAX8 [147], USF1 [148]. The top 4 downregulated transcription factors 
were: TEAD1 [149], MYC [150], THRA [151], TNNC1 [152]. Table 3 details these 14 factors along with their potential roles in disease and/or biological functions. Interestingly, several of these proteins have been identified as having roles in development and cancer or as factors associated with proliferation in hepatocytes.

\section{IPA analysis of differentially regulated proteins.}

Differentially regulated proteins were analyzed using the Ingenuity Pathway Analysis (IPA) program to elucidate trends and identify canonical functions. As expected given the functions of the most highly differentially regulated transcription factors, the top disease states identified were developmental disorders and cancer (Table 4). Gene expression, cellular growth and proliferation, cell death and survival, cellular development and cell cycle were all identified as the top molecular and cellular functions (Table 5), further supporting the potential roles of these factors in development and cancer. Among physiological system development, hematological and embryonic development were identified, as well as several other developmental functions (Table 6). Looking specifically within the liver functions, there are several proteins, which have been associated with hepatotoxicity, including: proliferation, cell death and hyperplasia (Figure 24). The top network identified by IPA was 
that of gene expression, cellular development and embryonic development (Table 7). Figure $\mathbf{2 5}$ details the interactions of differentially regulated transcription factors within this network. Taken together, our IPA analysis strongly suggests a potential role of PANDER within development and cancer.

PANDER expression in cell lines. To further investigate PANDER'S potential role in cancer, several cell lines were evaluated for PANDER protein expression via western blot. This blot is shown in Figure 26A. Interestingly, we see expression of PANDER in all investigated cell lines including: lung, ovarian, breast, pancreatic and bone. PANDER has not been studied with respect to these tissue types, with the exception of pancreatic, although PANDER was identified in the ovary in the tissue blot shown in Figure 13B. The ovarian cell lines are of particular interest because they include both noncancerous and cancerous cell lines. Densitometry analysis reveals that the relative expression of PANDER in all cancerous cell lines is decreased when compared to the noncancerous ovarian cell line (T80) (Figure 26B). This study provides supportive evidence suggesting that PANDER may play a role in suppressing the development or progression of ovarian and other cancers. 


\section{Discussion}

Significance of this study. This study provides the first analysis of a transcription factor profile in hepatocytes following treatment with pure secreted PANDER. Previous studies investigating PANDER stimulation have utilized either recombinant PANDER protein treatment or overexpression using an Ad-PANDER vector $[72,76]$, which could include both cleaved and uncleaved PANDER. This is the first study to use the pure secreted (cleaved) PANDER protein used to identify the crystal structure [64]. Given that PANDER is secreted from the pancreas and binds to its putative receptor on the liver cell membrane [84], it stands to reason that treatment of hepatocytes with secreted PANDER would provide the most biologically relevant snapshot of PANDER's functional effect on transcription factor activity. As such, the differentially regulated transcription factors identified in this study provide a unique and broadbased view into the potential functions of PANDER outside of its highly studied effects on metabolic signaling. In particular, we have identified a prospective role of PANDER in both cancer and development. The relationship between insulin resistance and cancer and PANDER's role in insulin resistance provide support for PANDER's function in cancer development and progression. PANDER's role in development has not been studied, although one array study identified FAM3B as being expressed in the 
endometrium of women recently implanted with an embryo and was dysregulated in women with unexplained infertility [153]. This study underscores the real need for further study of PANDER with respect to both cancer and embryonic development.

\section{Canonical networks effected by PANDER treatment. Ingenuity} Pathway Analysis identified several of the differentially regulated transcription factors associate with PANDER treatment as being involved with the gene expression, cellular development and embryonic development network (Figure 25). Many of the identified transcription factors play central roles within this network. CCAAT/enhancer-binding protein (CEBP) was the second most highly upregulated transcription factor in the PANDER treated hepatocytes and is highly involved with several other proteins within this network. CEBP is a transcription factor that has been implicated as both a tumor suppressor and a tumor promoter under different conditions in several cancers, due to its control of proliferative arrest and differentiation [154]. CEBP has also been identified as a regulator of the transcription of PEPCK [139, 155-157]. PEPCK is a gluconeogenic protein that has been previously shown to be upregulated in the PANDER transgenic overexpressor mouse [70] and downreguated in the PANDER knockout mouse [106]. This recent data regarding the upregulation of $\mathrm{CEBP}$ in response to PANDER treatment suggests 
that PANDER may be having an effect on PEPCK levels through a CEBP-dependent mechanism, and not solely through a regulation of insulin signaling. CEBP also plays an important role in proliferation arrest and differentiation of hepatocytes [158], as well as lipogenesis through regulation of SREBP-1c [159]. One of the most highly downregulated transcription factors in response to PANDER treatment was TEAD1 (TEF-1), which also plays a central role in the IPA identified top network, interacting with SP1, TNNC1, TBP and Smad2/3. TEAD1 is a part of the Hippo signaling pathway, which is associated with organ size control and tumor suppression through apoptosis [160]. In contrast, TEAD1 itself has been implicated as a biomarker for poor prognosis in prostate cancer [161] and as an activator of viral oncogenes [149, 162]. The downregulation of this protein in association with PANDER treatment further supports our initial data that suggest that PANDER may play a suppressive role in cancer. While there has been little direct research into PANDER's role in cancer, previous studies on PANDER's role in insulin resistance and apoptosis may provide insight into potential mechanisms.

Insulin resistance and cancer. Insulin resistance is characterized by the body's reduced ability to respond to insulin and suppress hepatic glucose production and stimulate 
glucose elimination [163]. As a result, blood glucose levels rise and, as part of a compensatory mechanism, insulin secretion increases to normalize glucose levels. This process leads to a hallmark of insulin resistance: hyperinsulinemia. Insulin resistance coincides with a number of conditions associated with an elevated risk of cancer, including obesity [164], central body fat distribution [165], physical inactivity [166], Type 2 diabetes [167], and hyperglycemia [168]. In addition, there has been extensive research linking characteristics associated with insulin resistance (in the absence of another concurrent disease) to the development and progression of cancer. Previous studies have shown that in rats treated with azoxymethane (a potent inducer of colon cancer) tumorigenesis was significantly enhanced by injection of insulin [169, 170]. Conversely, calorie restriction, an effective means to reduce insulin resistance, was shown to diminish the development of aberrant foci in the colon of these rats [171]. In human epidemiological studies, there has been a positive association identified between levels of insulin and/or c-peptide, both of which are measures of insulin resistance, and occurrence of pancreatic cancer [172, 173]. The mechanism by which insulin resistance and hyperinsulinemia promote cancer is not well understood, however, the downstream targets of the insulin signaling pathway reveal potential clues as to how insulin may be supporting cancer 
development. Insulin binding to the insulin receptor in the cell membrane activates two major pathways: MAPK and PI3K signaling pathways (Figure 27) [163]. The MAPK signaling pathway is involved in promoting cell survival and growth. PI3K signaling activates Akt, which, in turn, promotes cell proliferation and inhibits apoptosis and cell cycle arrest [163]. Both of these pathways are promoting cellular behaviors that are characteristic of cancer cells. Therefore, the hyperinsulinemia associated with the insulin resistant state may be promoting the development and progression of cancer though it's activation of the MAPK and PI3K pathways.

PANDER and insulin resistance. PANDER is localized to the insulin granules in $\beta$ cells, suggesting that it may be cosecreted with insulin. Pancreatic $\beta$ cell lines treated with glucose saw marked increases in both PANDER and insulin in a dose and time dependent manner [82]. In addition, chronic hyperglycemia associated with insulin resistance may activate PANDER expression and stimulate its cosecretion with insulin in pancreatic $\beta$ cells [107]. PANDER also has marked effects on the downstream targets of the insulin signaling pathway. PANDER has been shown to inhibit the insulin-stimulated activation of the PI3K pathway, including directly inhibiting the phosphorylation and subsequent activation of Akt (Figure 27) [84]. By inhibiting 
Akt, PANDER is effectively increasing the cell's ability to arrest the cell cycle and undergo apoptosis and decreasing cell proliferation. This suggests that PANDER may be inhibiting the cell's ability to undergo a transformation into a cancer cell. The MAPK signaling pathway, which induces cell growth and survival, has been shown to inhibit glucose-induced expression of PANDER. This indicates that, when hyperinsulinemia induces increased cell survival and growth through MAPK signaling, PANDER is subsequently decreased. This decrease in PANDER would then cause there to be a further increase in cell proliferation and anti-apoptotic effects though it's inability to inhibit Akt. This would suggest that the downregulation of PANDER may be necessary for the development of a cancerous phenotype, particularly in the insulin-resistant and hyperinsulinemic states.

\section{PANDER and p21.}

In addition to its role in the insulin signaling pathway, PANDER has been shown to induce apoptosis in primary pancreatic islet and islet cell lines. A study seeking to elucidate the mechanism behind this response showed that treatment with PANDER resulted in a significant downregulation of p21 in murine islets, eventually leading to cell death [76]. Cyclin-dependent kinase inhibitor $1 \mathrm{~A}$ (p21) is a cell cycle regulator and an important 
mediator of tumor suppressor p53 [174, 175]. Several studies have demonstrated that p21 expression can have an effect on the development and progression of cancer; however, the exact nature of this effect has varied. The function of p21 as a checkpoint protein suggests that it may have a proapoptotic (and anticancer) nature; several studies have supported this notion [176179]. Despite this, new studies suggest that p21 can be found at increased levels in tumors and metastases and that p21 deficiency can protect p53 mutant mice from developing tumors [180-183]. While the exact nature of PANDER and p21's interaction is unknown it is reasonable to surmise, given p21's potential role in both cancer development and in cancer suppression, that PANDER may also have some role in cancer as well.

PANDER and cancer. Given PANDER's ability to inhibit insulinstimulated PI3K and Akt activation and its association with p21 it is not hard to imagine that PANDER, itself, may play a role in the development and/or progression of cancer. New studies have shown PANDER to be downregulated in gastric cancer patients [184]. Another study identified PANDER to be the fourth most highly downregulated transcript in prostate cancer patients [185]. Preliminary data from our lab, in collaboration with Dr. Meera Nanjundan, suggest downregulation of PANDER among several 
ovarian cancer cell lines when compared to normal ovarian surface epithelial cells (Figure 26). A very similar result was identified in oral squamous carcinoma cell lines when compared to normal oral squamous cells [186]. Conversely, a non-secretory form of PANDER has been shown to increase invasion and migration in human colon cancer cells [187]. This suggests that PANDER my play a duel role in cancer pathogenesis or that secretory and non-secretory may serve different purposes. There have been no other studies to date investigating what role PANDER may be playing in cancer cell's ability to develop and proliferate. The role of PANDER in human cancers could be causative of cancer; it could be supportive of a previously established cancer; or it could even be inhibitory as part of the body's defense against cancer cells. PANDER's inhibition of known anti-apoptotic and pro-proliferative pathways suggests that PANDER may play an inhibitory role in cancer development and progression and that the downregulation of PANDER in patients promotes the growth and proliferation of cancer cells.

\section{Acknowledgements}

I would like to thank my mentor, Dr. Burkhardt, for his assistance in study design and data analysis. I would also like to thank Mark Athanason for his assistance with Ingenuity 
Pathway Analysis and Robert Hill for the use of his laboratory experiment. 
Biotin-labeled TF probes are mixed with nuclear extract

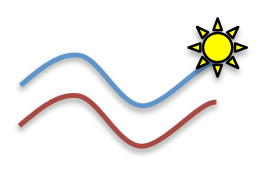

TF Probe 1

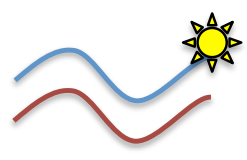

TF Probe 2

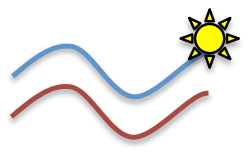

TF Probe 3

TFs present in extract bind probes

Columns separate unbound probe

Denaturation liberates free probe

Probes are hybridized to array containing TF consensus binding sequences
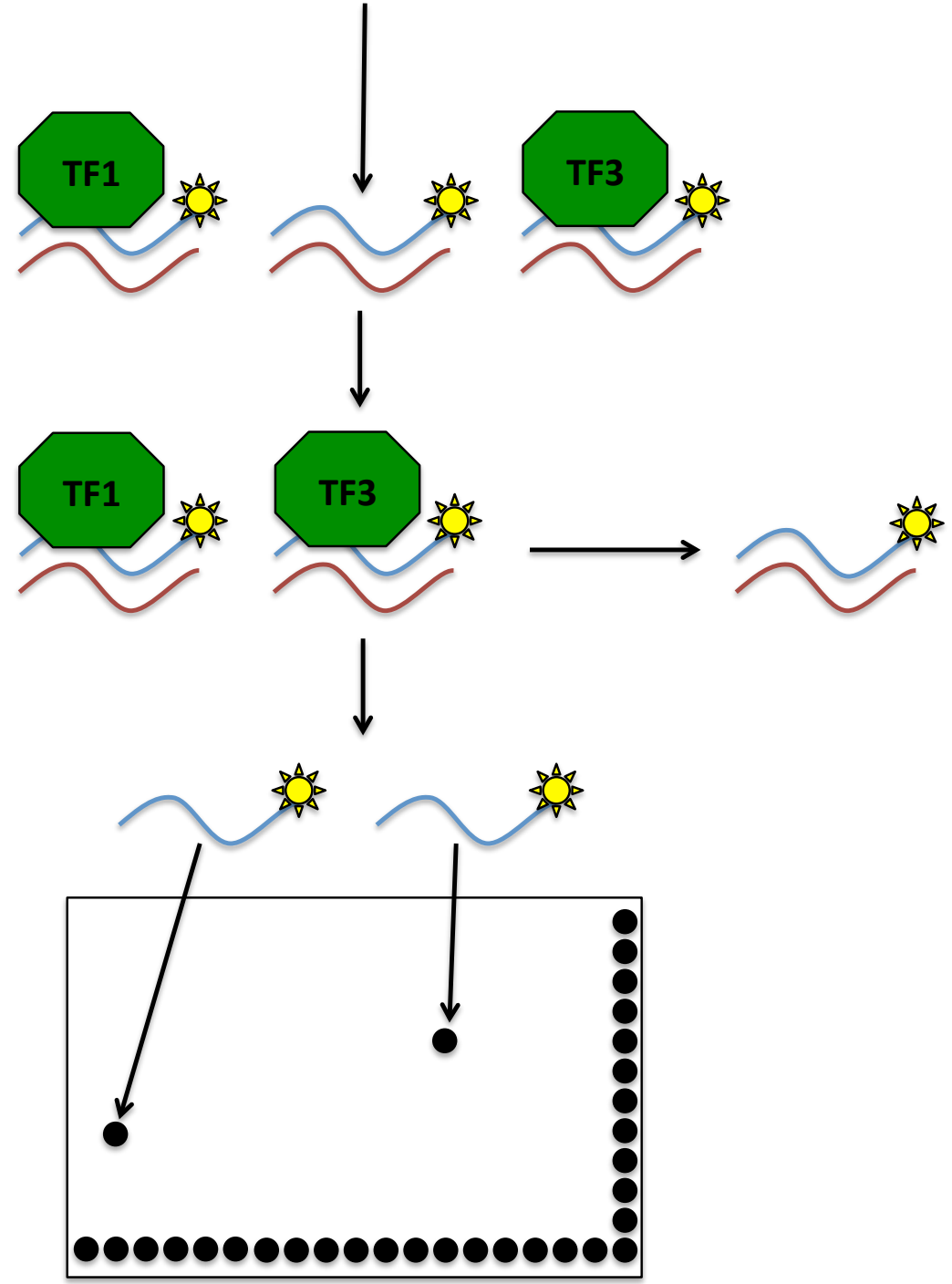

Figure 21: Overview of Transcription Factor Array. Biotinylated DNA oligonucleotide probes are mixed with hepatocyte nuclear extract. Transcription factors present in extract are then bound to probes and moved into columns where unbound probes are separated. Denaturation allows for the liberation of free probe, which is then hybridized to a membrane array containing consensus DNA binding sequences for 345 transcription factors. Biotin controls are located on the bottom and right of membrane to ensure equal distribution of extract. Adapted from Affymetrix (wWw.affymetrix.com). 


\section{ComboArray: Schematic Diagram of Protein/DNA ComboArray}

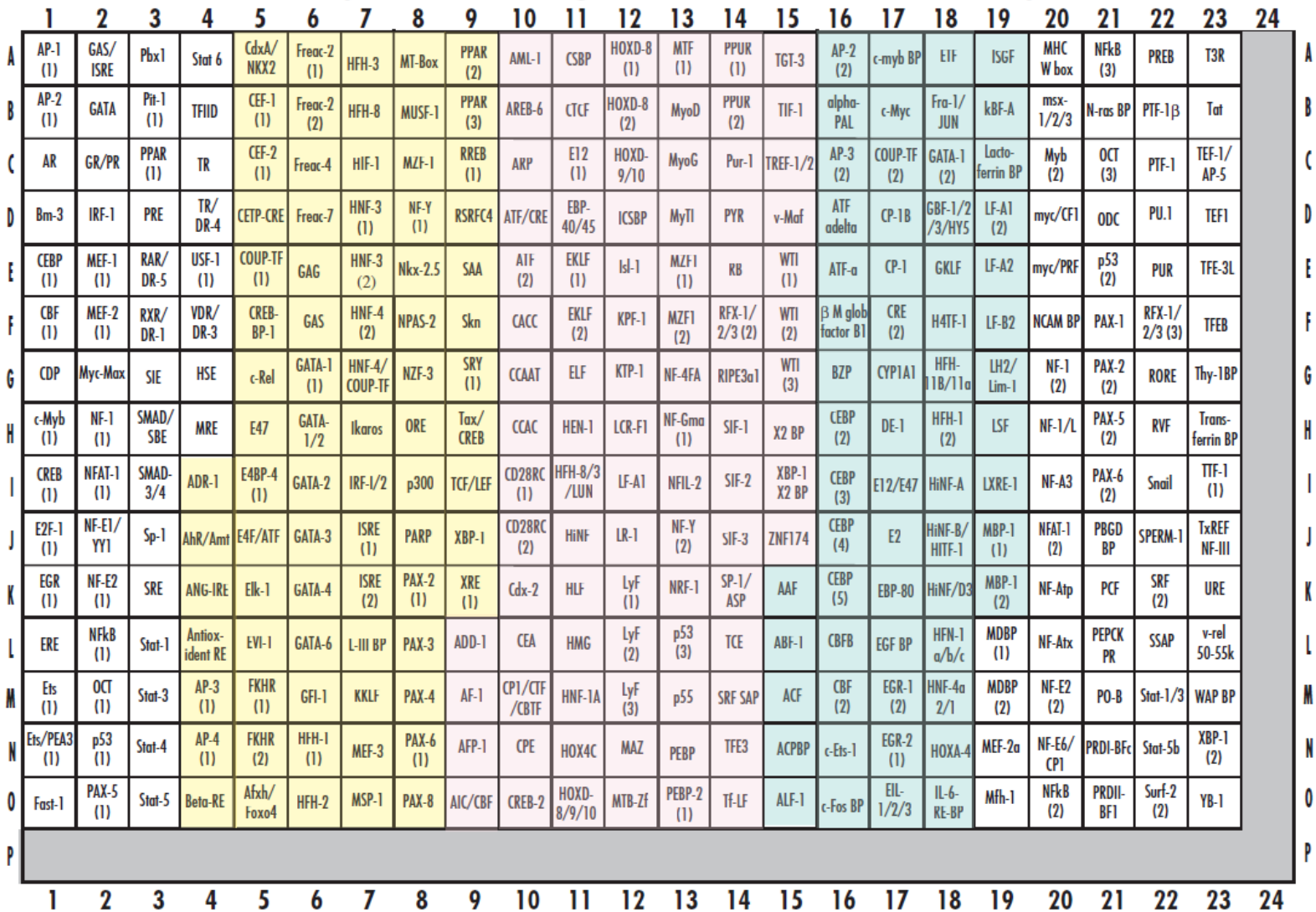

Figure 22: Diagram of Protein/DNA ComboArray from Affymetrix. The Panomics Protein/DNA ComboArray from Affymetrix is spotted with 345 transcription factor consensus binding sequences. The grey rows and columns contain the biotinylated DNA positive controls for the assay. 


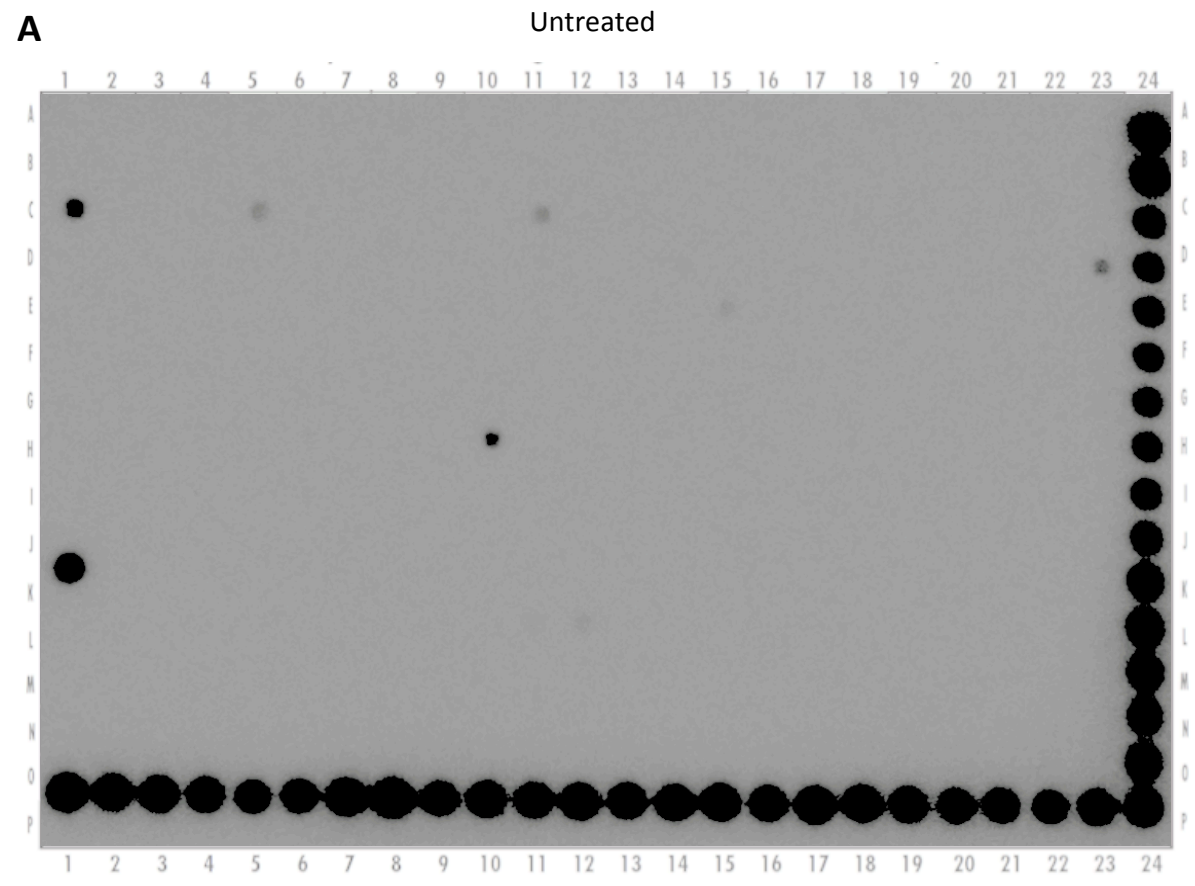

B

PANDER Treated

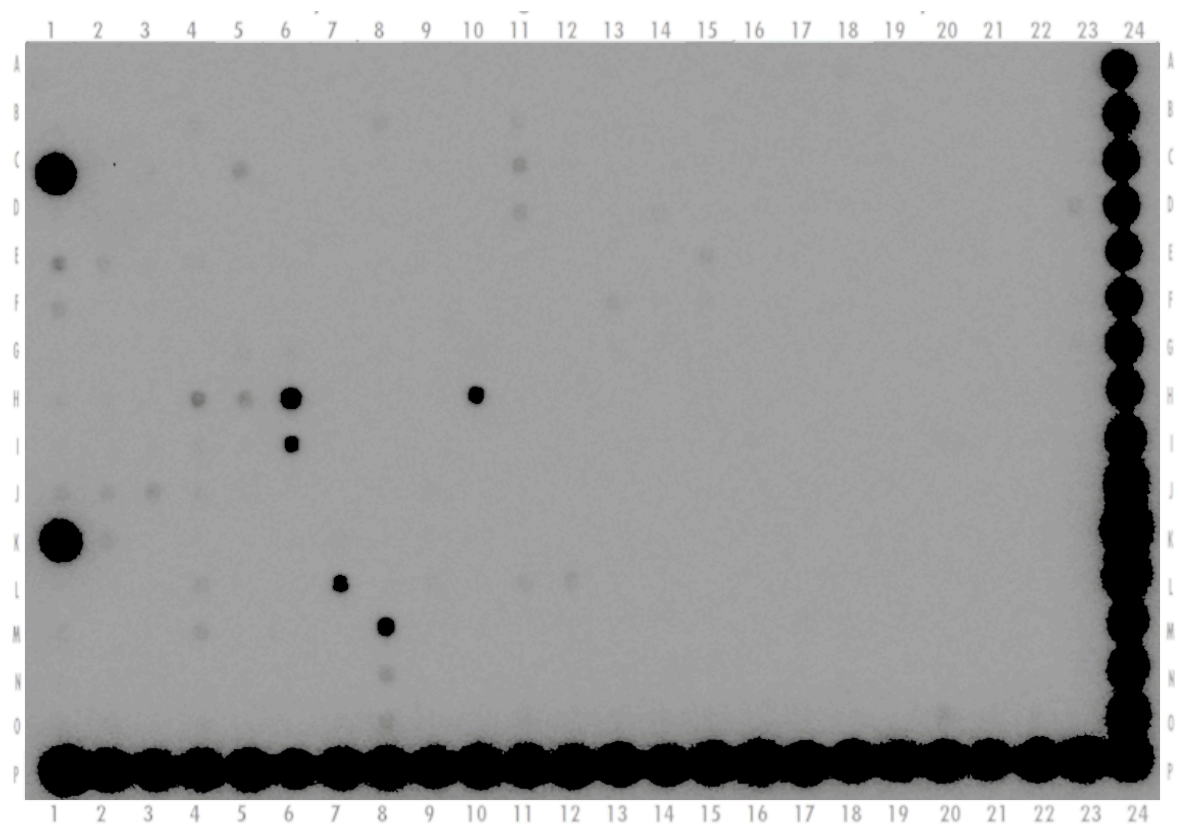

Figure 23: Transcription Factor Array membranes for untreated and pander treated hepatocytes. Untreated (A) and 10 nM PANDER treated (B) membranes are shown. Row $P$ and Column 24 represent biotinylated DNA positive controls. 
Table 2: Differentially regulated Transcription Factor Array.

\begin{tabular}{|l|r|}
\hline Protein & Fold Change \\
\hline GATA-2 & 1375.13 \\
\hline CEBP & 588.66 \\
\hline E47 & 442.92 \\
\hline PAX-6 & 371.69 \\
\hline Sp-1 & 367.88 \\
\hline AP-3 & 320.81 \\
\hline CBF & 276.47 \\
\hline MUSF-1 & 192.61 \\
\hline NF-E1/YY1 & 191.41 \\
\hline MEF-1 & 184.20 \\
\hline PAX-8 & 144.26 \\
\hline USF-1 & 125.00 \\
\hline TFIID & 114.96 \\
\hline AhR/Amt & 110.90 \\
\hline NFkB (2) & 101.48 \\
\hline PPAR & 83.81 \\
\hline C-Rel & 80.27 \\
\hline ISRE(2) & 79.97 \\
\hline XBP-1 & 75.77 \\
\hline ADR-1 & 71.97 \\
\hline RAR/DR-5 & 68.49 \\
\hline CCAAT & 68.31 \\
\hline EGR-1(2) & 67.67 \\
\hline MBP-1(1) & 63.77 \\
\hline PRE & 61.75 \\
\hline kBF-A & 59.71 \\
\hline ISGF & 57.16 \\
\hline NF-A3 & 55.09 \\
\hline E2F-1(1) & 54.25 \\
\hline TIF-I & 54.12 \\
\hline PAX-4 & 51.71 \\
\hline GATA-1/2 & 48.06 \\
\hline
\end{tabular}

\footnotetext{
**All data shown represent
} condition. proteins identified with

\begin{tabular}{|l|r|}
\hline Protein & Fold Change \\
\hline E4F/ATF & 32.08 \\
\hline Cdx-2 & 25.17 \\
\hline L-III BP & 24.23 \\
\hline WTI(2) & 22.81 \\
\hline MRE & 18.57 \\
\hline ETF & 17.27 \\
\hline MZF1 & 13.58 \\
\hline SMAD-3/4 & 8.99 \\
\hline Antioxident & 8.55 \\
\hline PAX-5 & 7.61 \\
\hline E2 & 6.00 \\
\hline EBP-40/45 & 4.55 \\
\hline Ets & 4.49 \\
\hline GATA-1 (1) & 4.26 \\
\hline c-Myb & 3.47 \\
\hline ADD-1 & 3.05 \\
\hline CTCF & 2.51 \\
\hline AR & 2.12 \\
\hline PYR & 2.08 \\
\hline CCAC & 1.46 \\
\hline HMG & 1.45 \\
\hline EGR & 1.35 \\
\hline LyF & 1.08 \\
\hline E12 & 0.98 \\
\hline CEF-2 & 0.82 \\
\hline WTI(1) & 0.76 \\
\hline TR & 0.40 \\
\hline MT-Box & 0.40 \\
\hline c-Myc & 0.34 \\
\hline TEF1 & 0.13 \\
\hline GATA-1 (2) & 0.04 \\
\hline
\end{tabular}

fold change from the untreated 
Table 3: Top differentially regulated proteins

\author{
Transcription Factor \\ GATA-2 \\ CEBPA \\ TCF3/E47 \\ PAX-6 \\ Sp-1 \\ YBX1/CBF \\ SAA1/MUSF-1 \\ NF-E1/YY1 \\ PAX-8 \\ USF-1 \\ TEAD1/TEF1 \\ $\mathrm{MYC} / \mathrm{c}-\mathrm{Myc}$ \\ THRA/TR \\ TNNC1/CEF-2
}

\section{Exp. Value}

1375.133

588.663

442.919

371.695

367.885

276.467

192.614

191.414

144.256

124.996

$-7.720$

$-2.905$

$-2.485$

$-1.215$

\section{Function}

Hematopoietic development Hepatocyte proliferation, gluconeogenesis Liver/Pancreatic development, Cancer CNS/Pancreatic developmemt

Hepatocyte proliferation

Cancer

Inflammation

DNA damage response, cancer

Embryonic development, cancer

Insulin signaling, lipogenesis

\author{
Cancer \\ Cancer \\ Development \\ Cardiac Muscle Contraction
}


Table 4: Top diseases and disorders identified by IPA

Diseases and Disorders

Name p-value

Developmental Disorder $\quad 7.02 \mathrm{E}-05-2.40 \mathrm{E}-13$

$\begin{array}{ll}\text { Cancer } & 7.07 \mathrm{E}-05-3.00 \mathrm{E}-11\end{array}$

Organismal Injury and Abnormalities

Gastrointestinal Disease

$7.07 \mathrm{E}-05-3.00 \mathrm{E}-11$

7.02E-05 - 1.10E-09

Hepatic System Disease

7.02E-05 - 1.10E-09

\#Molecules
24

37

37

16

15 
Table 5: Top molecular and cellular functions identified by IPA

Molecular and Cellular Functions

$\begin{array}{llc}\text { Name } & \text { p-value } & \text { \#Molecules } \\ \text { Gene Expression } & 7.28 \mathrm{E}-05-4.64 \mathrm{E}-35 & 41 \\ \text { Cellular Growth and Proliferation } & 6.15 \mathrm{E}-05-1.81 \mathrm{E}-20 & 42 \\ \text { Cell Death and Survival } & 6.04 \mathrm{E}-05-1.82 \mathrm{E}-19 & 40 \\ \text { Cellular Development } & 7.02 \mathrm{E}-05-2.96 \mathrm{E}-19 & 38 \\ \text { Cell Cycle } & 7.02 \mathrm{E}-05-3.27 \mathrm{E}-12 & 26\end{array}$




\section{Table 6: Top development functions identified by IPA}

Physiological System Development and Function

$\begin{array}{lc}\text { Name } & \text { p-value } \\ \text { Hematological System Development and Function } & 7.02 \mathrm{E}-05-5.78 \mathrm{E}-19\end{array}$

Organismal Survival

$2.38 \mathrm{E}-05-3.32 \mathrm{E}-17$

$6.92 \mathrm{E}-05-5.02 \mathrm{E}-16$

7.26E-05 - 5.02E-16

Organismal Development

7.02E-05 - 3.52E-15

\#Molecules

Hematopoiesis 
Analysis: PANDEREvalIPA - 2015-10-12 02:53 PM

- PANDEREvalIPA - 2015-10-12 02:53 PM

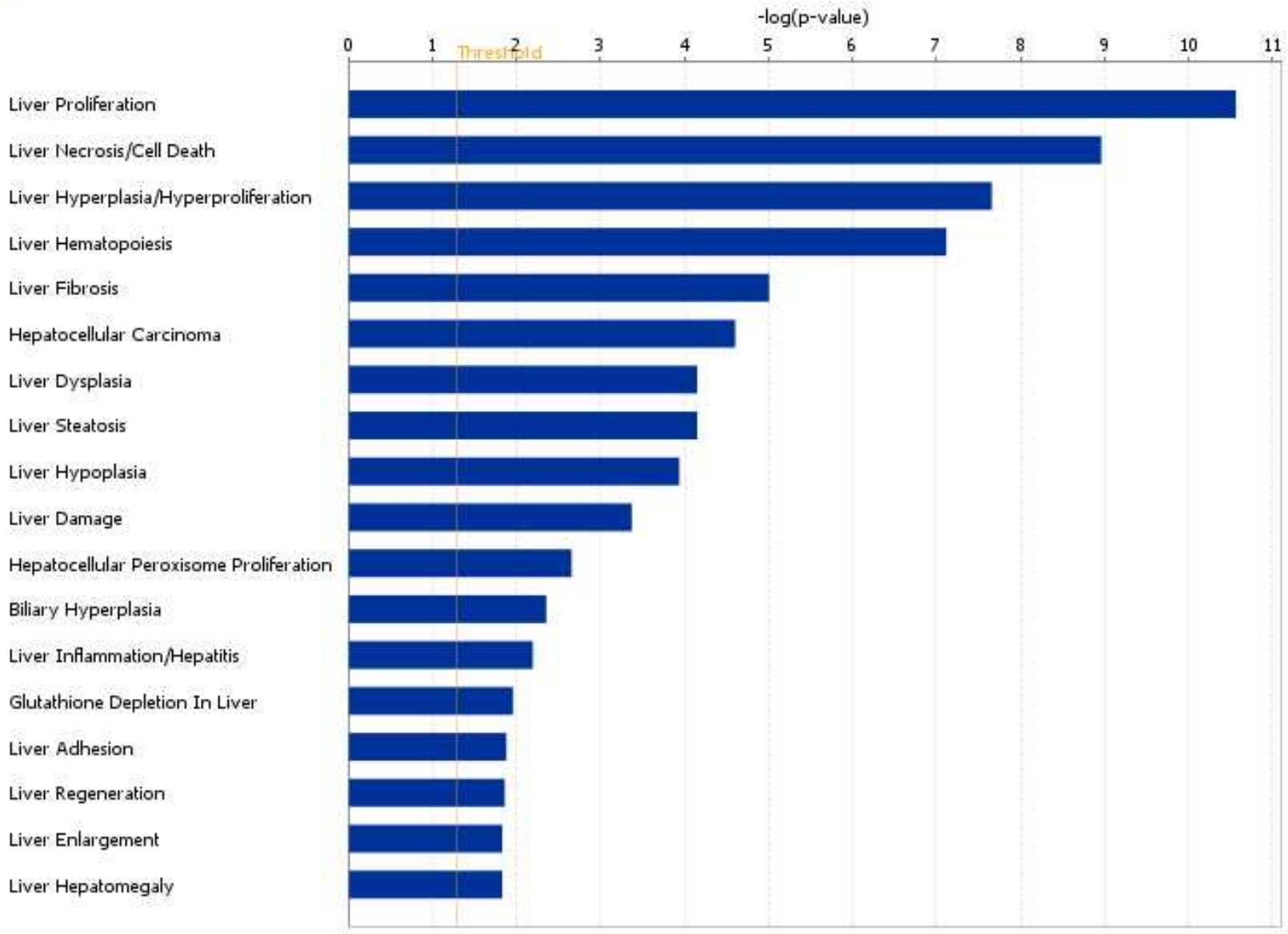

Q 2000-2015 QIAGEN. All rights reserved.

Figure 24: Top liver toxicity functions identified by IPA. IPA analysis identified several liver toxicity functions associated with the proteins that were differentially regulated in PANDER treated hepatocytes. 


\section{Table 7: Top networks identified by IPA}

\begin{tabular}{|c|c|c|}
\hline & Associated Network Functions & Score \\
\hline 1 & Gene Expression, Cellular Development, Embryonic Development & 28 \\
\hline 2 & Cellular Growth and Proliferation, Lymphoid Tissue Structure and Development, Cellular Development & 23 \\
\hline 3 & Gene Expression, Cellular Development, Cellular Growth and Proliferation & 23 \\
\hline 4 & Gene Expression, Carbohydrate Metabolism, Cellular Function and Maintenance & 13 \\
\hline & $\begin{array}{l}\text { Cell-To-Cell Signaling and Interaction, Hematological System Development and Function, Immune Cell } \\
\text { Trafficking }\end{array}$ & 7 \\
\hline
\end{tabular}


Path Designer Network 1

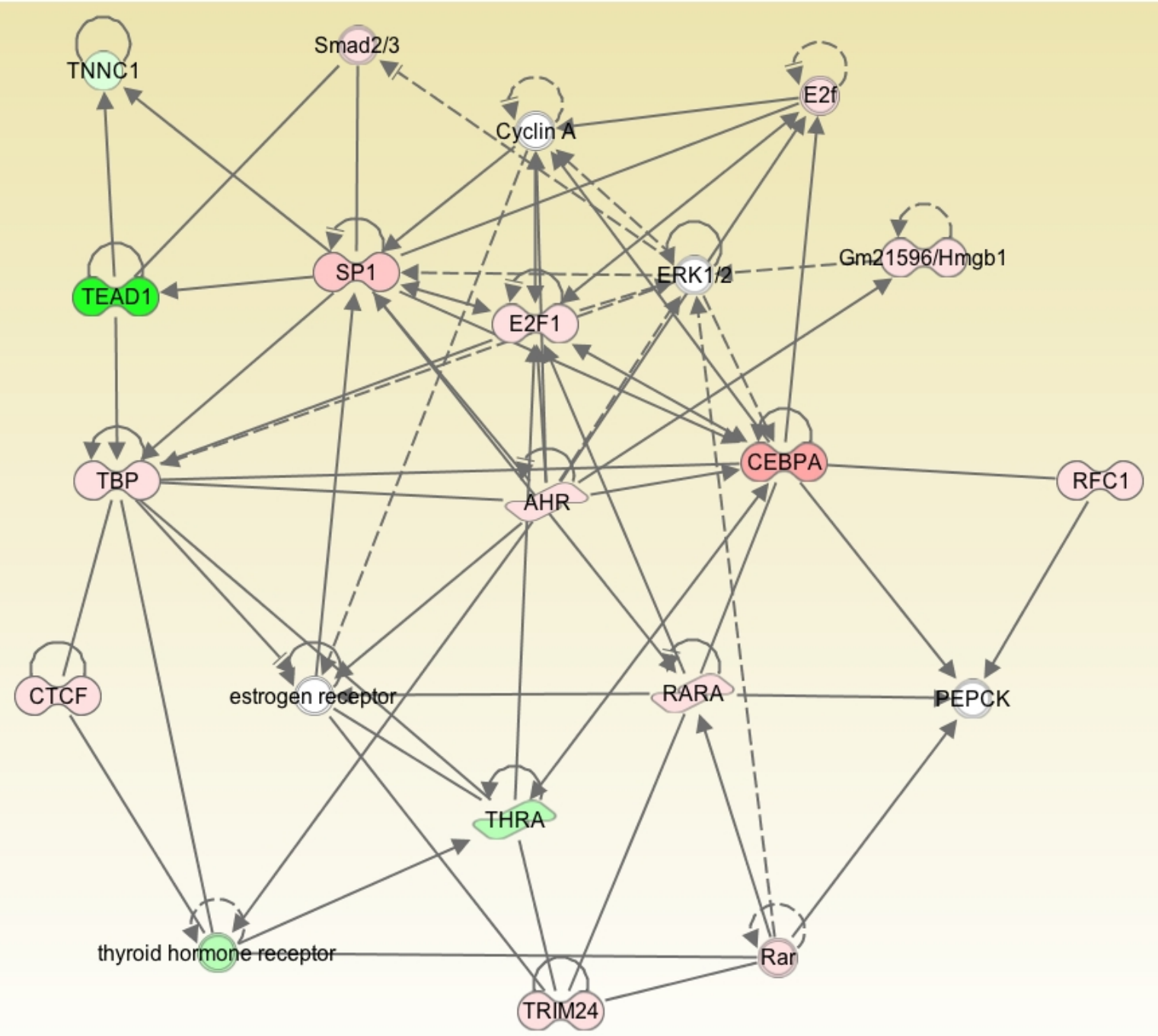

() 2000-2015 QIAGEN. All rights reserved.

Figure 25: Gene expression, cellular development and embryonic development network. Several differentially regulated proteins were found to be associated with the gene expression, cellular development and embryonic development network. These factors interact with each other as well as other important proteins, such as PEPCK and the estrogen receptor. Red indicates an upregulation in response to PANDER treatment and green indicates a downregulation in response to PANDER treatment. Proteins in white were not identified in the TF Array, but are important for functions within this network. 
A
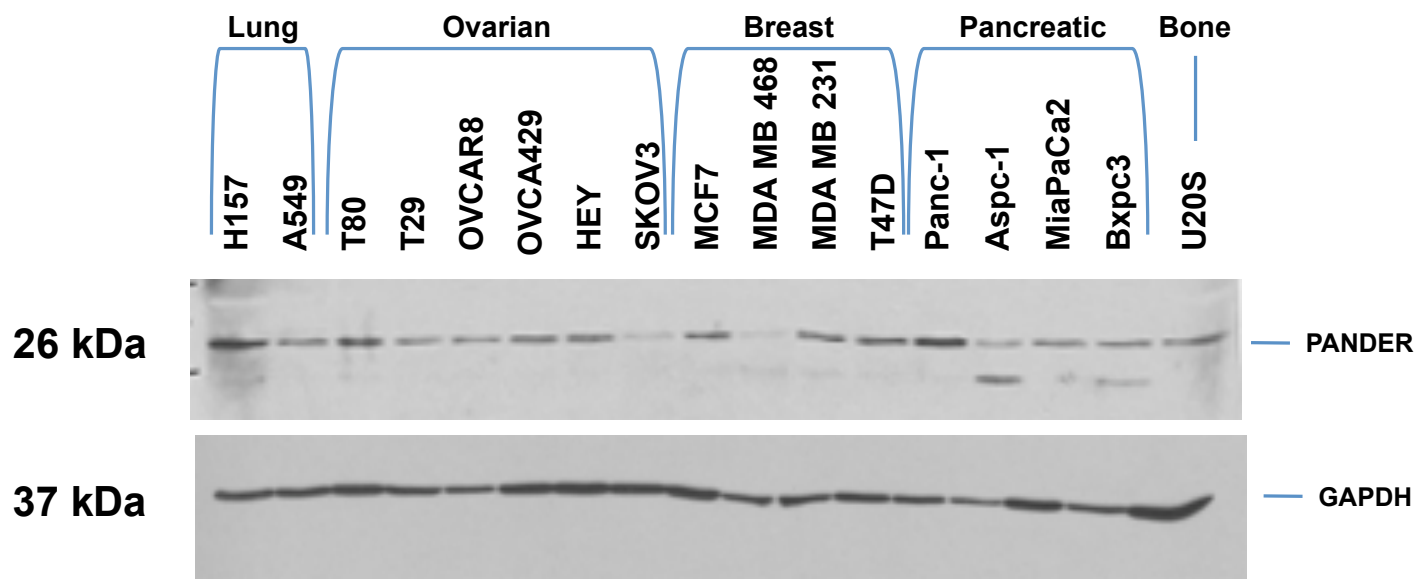

Figure 26: PANDER protein levels in ovarian cancer cell lines. (A) Western blot for PANDER in several cell lines performed in collaboration with Dr. Meera Nanjundan. (B) Densitometry performed on ovarian cell lines, noncancerous cell line shown in white, cancerous cell lines shown in black. 
B

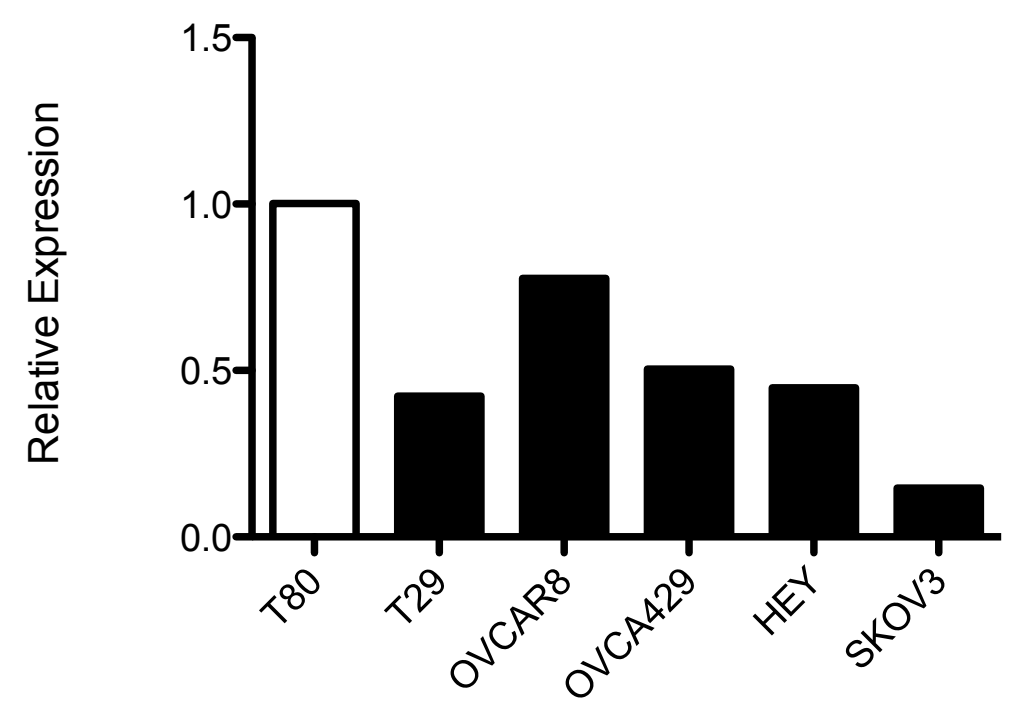

Figure 25: continued. 


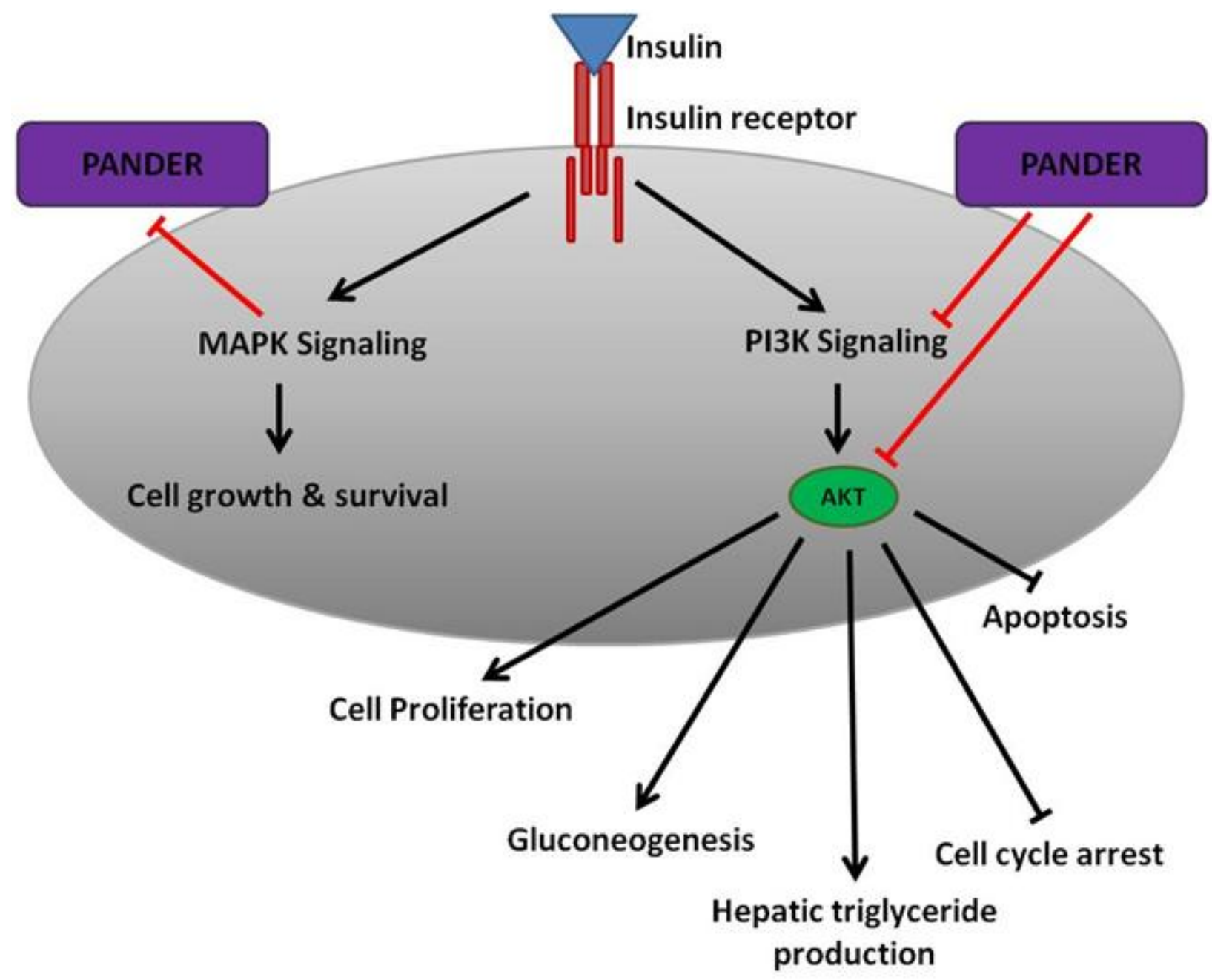

Figure 27: PANDER's activity within the insulin signaling pathway. The binding of insulin to the insulin receptor activates two major downstream pathways: MAPK and PI3K signaling pathways [163]. PANDER inhibits insulin-stimulated activation of the PI3K pathway, thereby reducing Akt's inhibition of apoptosis and cell cycle arrest and promotion of cell proliferation. MAPK signaling, which promotes cell growth and survival, has been shown to inhibit glucose-induced PANDER expression [84]. 


\section{CHAPTER 6: IMPLICATIONS AND FUTURE DIRECTIONS}

\section{Summary of Major Findings}

Type 2 Diabetes is characterized by insulin resistance, resulting in an overall state of hyperglycemia, hyperinsulinemia and hyperlipidemia [5]. Recent research has implicated several cytokines and other signaling molecules in contributing to the development and progression of this insulin resistant state [10]. Pancreatic-Derived Factor (PANDER) is one such molecule that has been identified as having a potential role in insulin resistance and T2D. The primary focus of previous PANDER research has been on its expression from and function within the pancreas; however, the liver has been identified as a novel tissue for expression [67]. This dissertation focuses primarily on the regulation of PANDER expression in the liver. As presented in chapter 3, we confirmed both the activity of the PANDER promoter and the presence of PANDER protein within liver tissues. We were also able to identify a novel transcriptional start site 26 bp upstream of the translational start codon. This is in contrast to the pancreatic start site, previously identified 520 bp upstream of the translational start codon 
[111]. This may suggest that there are differential mechanisms for the regulation of expression of PANDER within these two tissues. Further, we identified the minimal element for baseline promoter expression as being the $-293 /-3$ region, with the $-193 /-$ 93 region being essential for promoter activity. This region is also the minimal element responsible for the high level of glucose-stimulated activity of the PANDER promoter. Insulin and palmitate were not found to have any stimulatory effect. PANDER mRNA levels in primary hepatocytes mirror the promoter studies, significantly increasing in a dose-dependent manner with glucose treatment.

Within the identified minimal element of the PANDER promoter there are several putative transcription factor binding sites [118]. There are three E-Boxes within this region that are of particular interest as E-Boxes are known binding sites for Carbohydrate Responsive Element Binding Protein (ChREBP) [95]. ChREBP is a known glucose-responsive transcription factor within the liver and is involved in the activation of transcription of several genes associated with lipogenesis [99]. We investigated the impact of ChREBP expression on the PANDER promoter and found that when ChREBP is expressed in a liver cell line, PANDER promoter activity is upregulated in both the fasted and fed states. We have also identified the $-193 /-3$ region of the PANDER 
promoter as being bound by ChREBP under glucose-stimulatory conditions using chromatin immunoprecipitation in primary hepatocytes. There is only one intact E-box within that region: E-box 3. Site-directed mutagenesis was used to remove E-box 3 from the PANDER promoter. This mutant construct was significantly less active under baseline conditions and had a significantly reduced glucose-response. This suggests that E-box 3 is important for PANDER promoter activity, particularly under glucose-stimulatory conditions, and that this may be the specific site for ChREBP binding .

PANDER has not been studied in the liver with respect to its effect on transcription factor activity. In chapter 5, we utilized a transcription factor array and evaluated the response of 345 different transcription factors in primary hepatocytes with PANDER treatment. We found that there were 63 differentially regulated transcription factors: 55 were upregulated in response to PANDER treatment and 8 were downregulated. Ingenuity Pathway Analysis identified several of these transcription factors as having roles in cancer and/or development. Of particular interest was the significant upregulation of the CEBP transcription factor. CEBP has been shown to activate transcription of PEPCK [156]. PEPCK has been shown to be increased in the PANDER transgenic overexpressor 
mouse and decreased in the PANDER knockout mouse [70, 77]. PANDER's upregulation of CEBP is a novel finding and may implicate CEBP as an upstream factor resulting in the increased expression of PEPCK in the presence of elevated levels of PANDER •

\section{Chapter 3: Limitations of the Studies}

In Chapter 3 we investigated the regulation of PANDER in the liver by utilizing PANDER promoter constructs. In this respect, we are equating PANDER promoter activity with overall PANDER expression. We were able to confirm the association between PANDER promoter activity in response to glucose and PANDER mRNA expression in primary hepatocytes; however, we have not been able to confirm an upregulation of PANDER protein levels. This is primarily due to the lack of availability of reliable and specific PANDER antibodies for Western Blot or ELISA, particularly for cell culture lysate where protein concentration is low. We were able to show an increased trend of PANDER protein expression in response to glucose treatment in BNLCL2 cells via ELISA, however expression was below the range of the standard curve and could not be accurately analyzed (data not shown). Further development of commercial antibodies for PANDER is necessary to confirm these trend in protein. 


\section{Chapter 5: Limitations of the Studies}

Our TF array data provides some useful insight into the effects that PANDER has on transcription factor networks and disease; however, there are some limitations to this assay. Although protein concentration was normalized when applied to the membranes and exposure time was the same during imaging, there is no normalization control on the membrane itself to account for potential differences in transcription factor binding or exposure time to assay reagents. The positive controls on the membrane only serve as confirmation that the hybridization occurred evenly across the membrane. As such, minimal increases or decreases in transcription factor expression between the untreated and PANDER treated groups may be due to differences in membrane handling and not differences in expression. Due to this possibility, all of the identified factors should be validated by other means, such as RT-PCR or traditional western blot. In addition, further investigation into PANDER's expression in cancerous cell lines and tissues should be further evaluated based on the western blot showing decreased expression in cancerous versus normal ovarian epithelial cells to confirm the identified tend. 


\section{Future Directions}

The studies detailed in this dissertation have provided invaluable information regarding regulation of the PANDER promoter within the liver and how PANDER expression may be influencing transcription factor activity. This has provided a platform for future PANDER research; as there are several questions about PANDER that have yet to be addressed.

Human PANDER promoter activity. All of the previous studies investigating the activity of the PANDER promoter have focused on the murine PANDER promoter and murine cell lines. Although the murine and human PANDER genes share 78\% homology [110], it is unknown how the promoter may be differentially regulated in human tissues. As no human PANDER promoter constructs are known to exist, new constructs will need to be cloned into luciferase vectors and evaluated in a similar manner to how the murine promoter has been evaluated in the pancreas [111, 118] and in the liver [136].

ChREBP regulation of PANDER in the liver. Our studies showed that ChREBP binds to the PANDER promoter and increases its activity. We also identified a putative binding site for ChREBP at E-box 3. Further research is necessary to confirm E-box 3 as the binding site for ChREBP. Electrophoretic Mobility Shift 
Assay (EMSA) can be used to confirm the binding of ChREBP to the PANDER promoter and further narrow down its binding site. The PANDER mutant construct can also be used to determine if the exclusion of E-box 3 abrogates binding. In addition, further research is necessary to determine if E-boxes 1 and 2 are potential binding sites for ChREBP or other factors. To better understand ChREBP regulation of PANDER in an in vivo model, a ChREBP knockout mouse could be utilized. The ChREBP knockout mouse displays reduced lipogenesis and glycolysis [125, 131]; it would be interesting to see whether PANDER levels are also reduced when compared to WT mice.

PANDER regulation in other cell types. PANDER has been studied primarily in the pancreas and liver; however, data suggests that PANDER is produced within several tissues. The work included in this dissertation identified robust expression of PANDER protein in the murine brain and minor expression within the ovary. There have been no studies to date that have investigated the regulation or function of PANDER in these tissues. A similar promoter study to that performed in this dissertation could be performed in the brain and ovary, as well as other tissues to evaluate the activity and potential regulation of the PANDER promoter. The TF array data detailed in Chapter 5 suggests many potential functions for PANDER outside of its traditional role 
in metabolic signaling, which may speak to PANDER's function in tissues other than the pancreas and liver.

Other regulatory mechanisms of PANDER. Research into the regulation of PANDER has focused almost entirely on its regulation by transcription factors. There have been no major studies into how PANDER may be regulated by micro RNAs, histone modification or other epigenetic mechanisms. There has been a single abstract suggesting that the PANDER gene is silenced by epigenetic modification in gastric cancer cell lines, however, no future studies have been published based on this abstract [188]. We have demonstrated that PANDER has multiple cellspecific transcriptional start sites. One study found that transcription start sites that are located far downstream are more responsive to transcription factors, whereas upstream start sites may be regulated more by chromatin organization and other factors [121]. This suggests that while the liver-specific start site may be more highly regulated by transcription factors, while the pancreas-specific start site (520 bp upstream of the TSC) may be regulated by other factors.

CEBP, PEPCK and PANDER. Our transcription factor array identified 63 differentially regulated TFs in response to PANDER treatment. One of the most highly upregulated transcription 
factors in response to PANDER treatment was CEBP. CEBP has been shown to activate the transcription of PEPCK [156], a gluconeogenic protein that has also been shown to be upregulated in response to increased PANDER levels [70]. This suggests that perhaps CEBP represents an upstream factor in the PANDER activation of PEPCK and gluconeogenesis. Further research is necessary to validate the PANDER associated upregulation of CEBP and whether this is directly activating the transcription of PEPCK .

PANDER and cancer. Several of the differentially regulated transcription factors identified with our TF array appeared to be involved in cancer pathogenesis when analyzed using IPA. We also showed that PANDER protein levels appeared to be decreased in ovarian cancer cell lines when compared to a normal ovarian epithelial cell line. This result was very similar to the downregulation of PANDER that was identified in oral squamous carcinoma cell lines [186]. There are a number of studies that could be performed to further elucidate the role that PANDER may have in cancer, or its potential as a cancer biomarker. In vitro, cancer cells could be transiently transfected with a PANDER expression plasmid, similar to what was performed in human colon cancer cell lines [187]. These cells could then be evaluated for overall cell viability, invasion and migration. 
PANDER expression could also be silenced by creating an siRNA molecule specific to the PANDER gene. These cells could be similarly evaluated. In vivo, the PANDER knockout and transgenic mouse models could be utilized for tumor models to assess the viability of tumors when there is an absence or abundance of PANDER. PANDER's role in cancer could be evaluated under various conditions as well as with different cell/tumor types, as well.

PANDER receptor. While PANDER has been shown to bind to the liver cell membrane [84], its specific receptor remains unidentified. One method for identifying a PANDER receptor would be to prepare a plasmid cDNA library from liver cells. This plasmid library could then be transfected and expressed in a cell line that does not normally express the PANDER receptor. Expression of the PANDER receptor would be identified through binding of radiolabeled or fluorescently labeled PANDER protein. The receptor protein can then be identified by the cDNA that was expressed. Expression systems can then be used to synthesize receptor from its cDNA and study its properties.

PANDER as a therapeutic target for T2D. T2D is characterized by the progressive loss of glucose tolerance, resulting in prolonged hyperglycemia. Impaired glucose tolerance and fasting hyperglycemia are already present in many pre-diabetic patients 
and these factors represent strong risk factors for the eventual development of T2D [189]. Fasting hyperglycemia in T2D is also associated with an increase in hepatic glucose production [190]. Our PANDER transgenic mouse model displays a phenotype with multiple metabolic abnormalities that is similar to that of T2D [70]. In addition, patients with T2D appear to have higher circulating levels of PANDER [109]. Not only do diabetic patients display hyperglycemia, hyperinsulinemia and hyperlipidemia; they also appear to display hyperPANDERemia. This implicates PANDER as a potential therapeutic target for the treatment of T2D. Previous studies have shown that hepatic PANDER siRNA knockdown in $d b / d b$ mice significantly decreases hepatic steatosis, insulin resistance and fasting hyperglycemia [67]. These data suggest that a possible T2D therapeutic could be created to inhibit hepatic PANDER signaling, thereby decreasing HGP and improving insulin sensitivity • 


\section{REFERENCES}

1. Shaw, J.E., Sicree, R.A., and Zimmet, P.Z., Global estimates of the prevalence of diabetes for 2010 and 2030. Diabetes Res Clin Pract, 2010. 87(1): p. 4-14.

2. Centers for Disease Control and Prevention: National Diabetes Fact Sheet, 2011. 20116 October 2012]; Available from: http://www.cdc.gov/diabetes/pubs/factsheet11.htm.

3. Engelgau, M.M., Geiss, L.S., Saaddine, J.B., Boyle, J.P., Benjamin, S.M., Gregg, E.W., Tierney, E.F., Rios-Burrows, N., Mokdad, A.H., and Ford, E.S., The evolving diabetes burden in the United States. Annals of Internal Medicine, 2004 . $140(11)$ : p. 945 .

4. Songer, T.J., The economic costs of NIDDM. Diabetes/metabolism reviews, 2009. 8(4): p. 389-404.

5. Lin, Y. and Sun, Z., Current views on type 2 diabetes. Journal of Endocrinology, 2010. 204(1): p. 1-11.

6. Das, S.K. and Elbein, S.C., The genetic basis of type 2 diabetes. Cellscience, 2006. 2(4): p. 100.

7. Defronzo, R.A., Bonadonna, R.C., and Ferrannini, E., Pathogenesis of NIDDM: a balanced overview. Diabetes care, 1992. $15(3):$ p. 318-368.

8. Stumvoll, M., Goldstein, B.J., and van Haeften, T.W., Type 2 diabetes: principles of pathogenesis and therapy. The Lancet, 2005. 365(9467): p. 1333-1346.

9. Choi, S.M., Tucker, D.F., Gross, D.N., Easton, R.M., DiPilato, L.M., Dean, A.S., Monks, B.R., and Birnbaum, M.J., Insulin regulates adipocyte lipolysis via an Aktindependent signaling pathway. Molecular and cellular biology, 2010.30(21): p. 5009-5020.

10. Wang, C., Guan, Y. and Yang, J. Cytokines in the progression of pancreatic beta-cell dysfunction. Int $\mathrm{J}$ Endocrinol, 2010. DOI: 10.1155/2010/515136. 
11. Nov, O., Kohl, A., Lewis, E.C., Bashan, N., Dvir, I., BenShlomo, S., Fishman, S., Wueest, S., Konrad, D., and Rudich, A., Interleukin-lbeta may mediate insulin resistance in liver-derived cells in response to adipocyte inflammation. Endocrinology, 2010. 151(9): p. 4247-56.

12. Zhuang, X.F., Zhao, M.M., Weng, C.L., and Sun, N.L., Adipocytokines: a bridge connecting obesity and insulin resistance. Medical hypotheses, 2009. 73(6): p. 981-985.

13. Van Schaftingen, E. and Gerin, I., The glucose-6phosphatase system. Biochem. J, 2002. 362: p. 513-532.

14. Young, J., Gluconeogenesis in cattle: significance and methodology. Journal of dairy science, 1977. 60(1): p. 115 .

15. Michael, M.D., Kulkarni, R.N., Postic, C., Previs, S.F., Shulman, G.I., Magnuson, M.A., and Kahn, C.R., Loss of insulin signaling in hepatocytes leads to severe insulin resistance and progressive hepatic dysfunction. Molecular cell, 2000. 6(1): p. 87-97.

16. Edgerton, D.S., Lautz, M., Scott, M., Everett, C.A., Stettler, K.M., Neal, D.W., Chu, C.A., and Cherrington, A.D., Insulin's direct effects on the liver dominate the control of hepatic glucose production. Journal of Clinical Investigation, 2006. 116(2): p. 521.

17. Fritsche, L., Weigert, C., Haring, H.-U., and Lehmann, R., How insulin receptor substrate proteins regulate the metabolic capacity of the liver-implications for health and disease. Current medicinal chemistry, 2008. 15(13): p. 1316-1329.

18. Xu, E., Kumar, M., Zhang, Y., Ju, W., Obata, T., Zhang, N., Liu, S., Wendt, A., Deng, S., and Ebina, Y., Intra-islet insulin suppresses glucagon release via GABA-GABA A receptor system. Cell metabolism, 2006. 3(1): p. 47-58.

19. Duncan, R.E., Ahmadian, M., Jaworski, K., Sarkadi-Nagy, E., and Sul, H.S., Regulation of lipolysis in adipocytes. Annual review of nutrition, 2007. 27: p. 79. 
20. Rigazio, S., Lehto, H.-R., Tuunanen, H., Någren, K., Kankaanpaa, M., Simi, C., Borra, R., Naum, A.G., Parkkola, R., and Knuuti, J., The lowering of hepatic fatty acid uptake improves liver function and insulin sensitivity without affecting hepatic fat content in humans. American Journal of Physiology-Endocrinology and Metabolism, 2008. $295(2): p \cdot E 413-E 419$.

21. Boden, G., Chen, X., Capulong, E., and Mozzoli, M., Effects of free fatty acids on gluconeogenesis and autoregulation of glucose production in type 2 diabetes. Diabetes, 2001. $50(4):$ p. 810-816.

22. Sindelar, D.K., Chu, C.A., Rohlie, M., Neal, D.W., Swift, L.L., and Cherrington, A.D., The role of fatty acids in mediating the effects of peripheral insulin on hepatic glucose production in the conscious dog. Diabetes, 1997. $46(2): \mathrm{p} .187-196$.

23. Boden, G., Cheung, P., Stein, T.P., Kresge, K., and Mozzoli, M., FFA cause hepatic insulin resistance by inhibiting insulin suppression of glycogenolysis. American Journal of Physiology-Endocrinology and Metabolism, 2002 . $283(1)$ : p. E12-E19.

24. Griffin, M.E., Marcucci, M.J., Cline, G.W., Bell, K., Barucci, N., Lee, D., Goodyear, L.J., Kraegen, E.W., White, M.F., and Shulman, G.I., Free fatty acid-induced insulin resistance is associated with activation of protein kinase $C$ theta and alterations in the insulin signaling cascade. Diabetes, 1999. $48(6)$ : p. 1270-1274.

25. Boden, G., Role of fatty acids in the pathogenesis of insulin resistance and NIDDM. Diabetes, 1997. 46(1): p. 310 .

26. Marchesini, G., Brizi, M., Morselli-Labate, A.M., Bianchi, G., Bugianesi, E., McCullough, A.J., Forlani, G., and Melchionda, N., Association of nonalcoholic fatty liver disease with insulin resistance. The American journal of medicine, 1999. $107(5)$ : p. 450-455.

27. Miniño, A., Death in the United States, 2011. NCHS Data Brief, 115. Hyattsville, MD: National Center for Health Statistics, 2013. 
28. Browning, J.D., Szczepaniak, L.S., Dobbins, R., Horton, J.D., Cohen, J.C., Grundy, S.M., and Hobbs, H.H., Prevalence of hepatic steatosis in an urban population in the United States: impact of ethnicity. Hepatology, 2004. $40(6)$ : p. 1387-1395.

29. Clark, J.M., Brancati, F.L., and Diehl, A.M., The prevalence and etiology of elevated aminotransferase levels in the United States. The American journal of gastroenterology, 2003. 98(5): p. 960-967.

30. Ruhl, C. and Everhart, J., Fatty liver indices in the multiethnic United States National Health and Nutrition Examination Survey. Alimentary pharmacology \& therapeutics, 2015. $41(1):$ p. 65-76.

31. Clark, J.M. and Diehl, A.M., Nonalcoholic fatty liver disease: an underrecognized cause of cryptogenic cirrhosis. Jama, 2003. 289(22): p. 3000-3004.

32. Bugianesi, E., Moscatiello, S., Ciaravella, M., and Marchesini, G., Insulin resistance in nonalcoholic fatty liver disease. Current pharmaceutical design, 2010. 16(17): p. 1941-1951.

33. Chakrabarti, P. and V Kandror, K., Adipose triglyceride lipase: a new target in the regulation of lipolysis by insulin. Current diabetes reviews, 2011. 7(4): p. 270-277.

34. Jarrar, M., Baranova, A., Collantes, R., Ranard, B., Stepanova, M., Bennett, C., Fang, Y., Elariny, H., Goodman, Z., and Chandhoke, V., Adipokines and cytokines in nonalcoholic fatty liver disease. Alimentary pharmacology \& therapeutics, 2008. 27(5): p. 412-421.

35. Xu, H., Uysal, K.T., Becherer, J.D., Arner, P., and Hotamisligil, G.S., Altered tumor necrosis factor- $\alpha$ (TNF- $\alpha$ ) processing in adipocytes and increased expression of transmembrane TNF- $\alpha$ in obesity. Diabetes, 2002. 51(6): p. $1876-1883$.

36. Feldstein, A.E., Werneburg, N.W., Canbay, A., Guicciardi, M.E., Bronk, S.F., Rydzewski, R., Burgart, L.J., and Gores, G.J., Free fatty acids promote hepatic lipotoxicity by stimulating TNF- $\alpha$ expression via a lysosomal pathway. Hepatology, 2004. $40(1)$ : p. 185-194. 
37. Peraldi, P., Hotamisligil, G.S., Buurman, W.A., White, M.F., and Spiegelman, B.M., Tumor necrosis factor (TNF)- $\alpha$ inhibits insulin signaling through stimulation of the p55 TNF receptor and activation of sphingomyelinase. Journal of Biological Chemistry, 1996. 271(22): p. 13018-13022.

38. Uysal, K.T., Wiesbrock, S.M., Marino, M.W., and Hotamisligil, G.S., Protection from obesity-induced insulin resistance in mice lacking TNF- $\alpha$ function. Nature, 1997. $389(6651)$ : p. 610-614.

39. Valenti, L., Fracanzani, A.L., Dongiovanni, P., Santorelli, G., Branchi, A., Taioli, E., Fiorelli, G., and Fargion, S., Tumor necrosis factor a promoter polymorphisms and insulin resistance in nonalcoholic fatty liver disease. Gastroenterology, 2002. $122(2)$ : p. 274-280.

40. Hirosumi, J., Tuncman, G., Chang, L., Görgün, C.Z., Uysal, K.T., Maeda, K., Karin, M., and Hotamisligil, G.S., A central role for JNK in obesity and insulin resistance. Nature, 2002. $420(6913):$ p. 333-336.

41. Wieckowska, A., Papouchado, B.G., Li, Z., Lopez, R., Zein, N.N., and Feldstein, A.E., Increased hepatic and circulating interleukin-6 levels in human nonalcoholic steatohepatitis. The American journal of gastroenterology, 2008. $103(6)$ : p. 1372-1379.

42. Bugianesi, E., Pagotto, U., Manini, R., Vanni, E., Gastaldelli, A., de Iasio, R., Gentilcore, E., Natale, S., Cassader, M., and Rizzetto, M., Plasma adiponectin in nonalcoholic fatty liver is related to hepatic insulin resistance and hepatic fat content, not to liver disease severity. The Journal of Clinical Endocrinology \& Metabolism, 2005.90(6): p. 3498-3504.

43. Chu, C.-J., Lu, R.-H., Wang, S.-S., Chang, F.-Y., Lin, S.Y., Yang, C.-Y., Lin, H.-C., Chang, C.-Y., Wu, M.-Y., and Lee, S.-D., Plasma levels of interleukin-6 and interleukin8 in Chinese patients with non-alcoholic fatty liver disease. Hepato-gastroenterology, 2006. 54(79): p. 20452048 .

44. Engelgau, M.M., Geiss, L.S., Saaddine, J.B., Boyle, J.P., Benjamin, S.M., Gregg, E.W., Tierney, E.F., Rios-Burrows, N., Mokdad, A.H., and Ford, E.S., The evolving diabetes burden in the United States. Annals of internal medicine, 2004. 140(11): p. 945-950. 
45. Unwin, N., Gan, D., and Whiting, D., The IDF Diabetes Atlas: providing evidence, raising awareness and promoting action. Diabetes research and clinical practice, 2010. $87(1): p \cdot 2-3$

46. Imperatore, G., Boyle, J.P., Thompson, T.J., Case, D., Dabelea, D., Hamman, R.F., Lawrence, J.M., Liese, A.D., Liu, L.L., and Mayer-Davis, E.J., Projections of Type 1 and Type 2 Diabetes Burden in the US Population Aged< 20 Years Through 2050 Dynamic modeling of incidence, mortality, and population growth. Diabetes Care, 2012. 35(12): p. 25152520 .

47. Patterson, C.C., Dahlquist, G.G., Gyürüs, E., Green, A., Soltész, G., and Group, E.S., Incidence trends for childhood type 1 diabetes in Europe during 1989-2003 and predicted new cases 2005-20: a multicentre prospective registration study. The Lancet, 2009. 373(9680): p. 20272033 .

48. Vehik, K., Ajami, N.J., Hadley, D., Petrosino, J.F., and Burkhardt, B.R., The changing landscape of type 1 diabetes: recent developments and future frontiers. Current diabetes reports, 2013. 13(5): p. 642-650.

49. Bluestone, J.A., Herold, K., and Eisenbarth, G., Genetics, pathogenesis and clinical interventions in type [thinsp] 1 diabetes. Nature, 2010.464(7293): p. 1293-1300.

50. Laakso, M. and Pyörälä, K., Age of onset and type of diabetes. Diabetes care, 1985. 8(2): p. 114-117.

51. Strachan, D.P., Hay fever, hygiene, and household size. Bmj, 1989. 299(6710): p. 1259-1260.

52. Kolb, $\mathrm{H}$. and Elliott, R., Increasing incidence of IDDM a consequence of improved hygiene? Diabetologia, 1994. 37(7): p. $729-729$.

53. Dahlquist, G., Can we slow the rising incidence of childhood-onset autoimmune diabetes? The overload hypothesis. Diabetologia, 2006. $49(1)$ : p. 20-24.

54. Barrett, J.C., Clayton, D.G., Concannon, P., Akolkar, B., Cooper, J.D., Erlich, H.A., Julier, C., Morahan, G., Nerup, J., and Nierras, C., Genome-wide association study and meta-analysis find that over 40 loci affect risk of type 1 diabetes. Nature genetics, 2009. 41(6): p. 703-707. 
55. Todd, J.A., Walker, N.M., Cooper, J.D., Smyth, D.J., Downes, K., Plagnol, V., Bailey, R., Nejentsev, S., Field, S.F., and Payne, F., Robust associations of four new chromosome regions from genome-wide analyses of type 1 diabetes. Nature genetics, 2007. 39(7): p. 857-864.

56. Zhang, A., Sun, H., and Wang, X., Serum metabolomics as a novel diagnostic approach for disease: a systematic review. Analytical and bioanalytical chemistry, 2012. 404(4): p. $1239-1245$.

57. Sysi-Aho, M., Ermolov, A., Gopalacharyulu, P.V., Tripathi, A., Seppanen-Laakso, T., Maukonen, J., Mattila, I., Ruohonen, S.T., Vahatalo, L., and Yetukuri, L., Metabolic regulation in progression to autoimmune diabetes. PLos Comput Biol, 2011. 7(10): p. e1002257.

58. Atkinson, M.A. and Eisenbarth, G.S., Type 1 diabetes: new perspectives on disease pathogenesis and treatment. The Lancet, 2001. 358(9277): p. 221-229.

59. Matsushima, M., Laporte, R., Maruyama, M., Shimizu, K., Nishimura, R., Tajima, N., and Group, D.M.S., Geographic variation in mortality among individuals with youth-onset diabetes mellitus across the world. Diabetologia, 1997.

$40(2): \mathrm{p} .212-216$.

60. Podar, T., Solntsev, A., Reunanen, A., Urbonaite, B., Zalinkevicius, R., Karvonen, M., LaPorte, R.E., and Tuomilehto, J., Mortality in patients with childhood-onset type 1 diabetes in Finland, Estonia, and Lithuania: followup of nationwide cohorts. Diabetes Care, 2000. 23(3): p. 290-294.

61. Aurora, R. and Rose, G.D., Seeking an ancient enzyme in Methanococcus jannaschii using ORF, a program based on predicted secondary structure comparisons. Proceedings of the National Academy of Sciences, 1998. 95(6): p. 28182823 .

62. Abdel-Meguid, S.S., Shieh, H.S., Smith, W.W., Dayringer, H.E., Violand, B.N., and Bentle, L.A., Three-dimensional structure of a genetically engineered variant of porcine growth hormone. Proceedings of the National Academy of Sciences, 1987. 84(18): p. 6434-6437. 
63. Wang, O., Cai, K., Pang, S., Wang, T., Qi, D., Zhu, Q., Ni, Z., and Le, Y., Mechanisms of glucose-induced expression of pancreatic-derived factor in pancreatic beta-cells. Endocrinology, 2008. $149(2)$ : p. 672-80.

64. Johansson, P., Bernstrom, J., Gorman, T., Oster, L., Backstrom, S., Schweikart, F., Xu, B., Xue, Y., and Schiavone, L.H., FAM3B PANDER and FAM3C ILEI represent a distinct class of signaling molecules with a non-cytokinelike fold. Structure, 2013. 21(2): p. 306-13.

65. Zhu, Y., Xu, G., Patel, A., McLaughlin, M.M., Silverman, C., Knecht, K.A., Sweitzer, S., Li, X., McDonnell, P., and Mirabile, R., Cloning, expression, and initial characterization of a novel cytokine-like gene family. Genomics, 2002. 80(2): p. 144-150.

66. Burkhardt, B.R., Yang, M.C., Robert, C.E., Greene, S.R., McFadden, K.K., Yang, J., Wu, J., Gao, Z., and Wolf, B.A., Tissue-specific and glucose-responsive expression of the pancreatic derived factor (PANDER) promoter. Biochimica et biophysica acta, 2005. $1730(3)$ : p. 215-25.

67. Li, J., Chi, Y., Wang, C., Wu, J., Yang, H., Zhang, D., Zhu, Y., Wang, N., Yang, J., and Guan, Y., Pancreaticderived factor promotes lipogenesis in the mouse liver: Role of the Forkhead box 1 signaling pathway. Hepatology, 2011. $53(6)$ : p. 1906-1916.

68. Cao, X., Gao, Z., Robert, C.E., Greene, S., Xu, G., Xu, W., Bell, E., Campbell, D., Zhu, Y., and Young, R., Pancreaticderived factor (FAMBB), a novel islet cytokine, induces apoptosis of insulin-secreting $\beta$-cells. Diabetes, 2003. $52(9)$ : p. 2296-2303.

69. Wilson, C.G., Schupp, M., Burkhardt, B.R., Wu, J., Young, R.A., and Wolf, B.A., Liver-specific overexpression of pancreatic-derived factor (PANDER) induces fasting hyperglycemia in mice. Endocrinology, 2010. 151(11): p. 5174-84.

70. Robert-Cooperman, C.E., Dougan, G.C., Moak, S.L., Athanason, M.G., Kuehl, M.N., Bell-Temin, H., Stevens, S.M., and Burkhardt, B.R., PANDER transgenic mice display fasting hyperglycemia and hepatic insulin resistance. Journal of Endocrinology, 2014. 220(3): p. 219-231. 
71. Moak, S.L., Dougan, G.C., MarElia, C.B., Danse, W.A., Fernandez, A.M., Kuehl, M.N., Athanason, M.G., and Burkhardt, B.R., Enhanced glucose tolerance in pancreatic derived factor (PANDER) knockout C57BL/6 mice. Dis Model Mech, 2014 .

72. Cao, X., Yang, J., Burkhardt, B.R., Gao, Z., Wong, R.K., Greene, S.R., Wu, J., and Wolf, B.A., Effects of overexpression of pancreatic derived factor (FAMBB) in isolated mouse islets and insulin-secreting $\beta T C 3$ cells. American Journal of Physiology-Endocrinology And Metabolism, 2005. 289(4): p. E543-E550.

73. Detjen, K.M., Murphy, D., Welzel, M., Farwig, K., Wiedenmann, B., and Rosewicz, S., Downregulation of p2lwaf/cip-1 mediates apoptosis of human hepatocellular carcinoma cells in response to interferon-r. Experimental cell research, 2003. 282(2): p. 78-89.

74. Liadis, N., Murakami, K., Eweida, M., Elford, A.R., Sheu, L., Gaisano, H.Y., Hakem, R., Ohashi, P.S., and Woo, M., Caspase-3-dependent $\beta$-cell apoptosis in the initiation of autoimmune diabetes mellitus. Molecular and Cellular Biology, 2005. 25(9): p. 3620-3629.

75. Jin, Y.H., Yoo, K.J., Lee, Y.H., and Lee, S.K., Caspase 3mediated cleavage of p21 WAFI/CIPI associated with the cyclin A-cyclin-dependent kinase 2 complex is a prerequisite for apoptosis in SK-HEP-1 cells. Journal of Biological Chemistry, 2000. 275(39): p. 30256-30263.

76. Burkhardt, B.R., Greene, S.R., White, P., Wong, R.K., Brestelli, J.E., Yang, J., Robert, C.E., Brusko, T.M., Wasserfall, C.H., Wu, J., Atkinson, M.A., Gao, Z., Kaestner, K.H., and Wolf, B.A., PANDER-induced cell-death genetic networks in islets reveal central role for caspase3 and cyclin-dependent kinase inhibitor $1 \mathrm{~A}$ (p21). Gene, 2006. 369: p. 134-41.

77. Robert-Cooperman, C.E., Carnegie, J.R., Wilson, C.G., Yang, J., Cook, J.R., Wu, J., Young, R.A., Wolf, B.A., and Burkhardt, B.R., Targeted disruption of pancreatic-derived factor (PANDER, FAM3B) impairs pancreatic beta-cell function. Diabetes, 2010. 59(9): p. 2209-18. 
78. Marie, J., Bailbe, D., Gylfe, E., and Portha, B., Defective glucose-dependent cytosolic Ca2t handling in islets of GK and nSTZ rat models of type 2 diabetes. Journal of endocrinology, 2001. 169(1): p. 169-176.

79. Giroix, M.-H., Sener, A., Bailbe, D., Leclercqmeyer, V., Portha, B., and Malaisse, W., Metabolic, ionic, and secretory response to D-glucose in islets from rats with acquired or inherited non-insulin-dependent diabetes. Biochemical medicine and metabolic biology, 1993. 50(3): p. $301-321$.

80. Roe, M.W., Philipson, L.H., Frangakis, C.J., Kuznetsov, A., Mertz, R.J., Lancaster, M.E., Spencer, B., Worley, J.F., and Dukes, I.D., Defective glucose-dependent endoplasmic reticulum Ca2t sequestration in diabetic mouse islets of Langerhans. Journal of Biological Chemistry, 1994. 269(28): p. 18279-18282.

81. Xu, W., Gao, Z., Wu, J., and Wolf, B.A., Interferon-rinduced regulation of the pancreatic derived cytokine FAMBB in islets and insulin-secreting BTC3 cells. Molecular and cellular endocrinology, 2005. $240(1)$ : p. 74-81.

82. Yang, J., Robert, C.E., Burkhardt, B.R., Young, R.A., Wu, J., Gao, Z., and Wolf, B.A., Mechanisms of glucose-induced secretion of pancreatic-derived factor (PANDER or FAM3B) in pancreatic $\beta$-cells. Diabetes, 2005. 54(11): p. 3217-3228.

83. Carnegie, J.R., Robert-Cooperman, C.E., Wu, J., Young, R.A., Wolf, B.A., and Burkhardt, B.R., Characterization of the expression, localization, and secretion of PANDER in alpha-cells. Mol Cell Endocrinol, 2010. 325(1-2): p. 36-45.

84. Yang, J., Wang, C., Li, J., Burkhardt, B.R., RobertCooperman, C.E., Wilson, C., Gao, Z., and Wolf, B.A., PANDER binds to the liver cell membrane and inhibits insulin signaling in HepG2 cells. FEBS Lett, 2009. 583(18): p. 3009-15.

85. Monetti, M., Nagaraj, N., Sharma, K., and Mann, M., Largescale phosphosite quantification in tissues by a spike-in SILAC method. Nature methods, 2011. 8(8): p. 655-658.

86. Liu, X. and Gorovsky, M.A., Mapping the 5' and 3' ends of Tetrahymena thermophila mRNAs using RNA ligase mediated amplification of $C D N A$ ends (RLM-RACE). Nucleic acids research, 1993. 21(21): p. 4954-4960. 
87. Burkhardt, B.R., Cook, J.R., Young, R.A., and Wolf, B.A., PDX-1 interaction and regulation of the Pancreatic Derived Factor (PANDER, FAM3B) promoter. Biochimica et biophysica acta, 2008. $1779(10)$ : p. 645-51.

88. Saltiel, A.R. and Kahn, C.R., Insulin signalling and the regulation of glucose and lipid metabolism. Nature, 2001. $414(6865):$ p. 799-806.

89. Whiteman, E.L., Cho, H., and Birnbaum, M.J., Role of Akt/protein kinase $B$ in metabolism. Trends in Endocrinology \& Metabolism, 2002. 13(10): p. 444-451.

90. Tzivion, G., Dobson, M., and Ramakrishnan, G., FoxO transcription factors; Regulation by AKT and 14-3-3 proteins. Biochimica et Biophysica Acta (BBA)-Molecular Cell Research, 2011. 1813(11): p. 1938-1945.

91. Kim, S.-Y., Kim, H.-i., Kim, T.-H., Im, S.-S., Park, S.-K., Lee, I.-K., Kim, K.-S., and Ahn, Y.-H., SREBP-IC mediates the insulin-dependent hepatic glucokinase expression. Journal of Biological Chemistry, 2004. 279(29): p. 3082330829 .

92. Foretz, M., Pacot, C., Dugail, I., Lemarchand, P., Guichard, C., le Lièpvre, X., Berthelier-Lubrano, C., Spiegelman, B., Kim, J.B., and Ferré, P., ADD1/SREBP-1C is required in the activation of hepatic lipogenic gene expression by glucose. Molecular and cellular biology, 1999. $19(5):$ p. 3760-3768.

93. Yamamoto, T., Shimano, H., Nakagawa, Y., Ide, T., Yahagi, N., Matsuzaka, T., Nakakuki, M., Takahashi, A., Suzuki, H., and Sone, H., SREBP-1 interacts with hepatocyte nuclear factor $-4 \alpha$ and interferes with $P G C-1$ recruitment to suppress hepatic gluconeogenic genes. Journal of Biological Chemistry, 2004.279(13): p. 12027-12035.

94. Dentin, R., Pégorier, J.-P., Benhamed, F., Foufelle, F., Ferré, P., Fauveau, V., Magnuson, M.A., Girard, J., and Postic, C., Hepatic glucokinase is required for the synergistic action of ChREBP and SREBP-IC on glycolytic and lipogenic gene expression. Journal of Biological Chemistry, 2004. $279(19)$ : p. 20314-20326. 
95. Dentin, R., Girard, J., and Postic, C., Carbohydrate responsive element binding protein (ChREBP) and sterol regulatory element binding protein-1C (SREBP-1C): two key regulators of glucose metabolism and lipid synthesis in liver. Biochimie, 2005. $87(1)$ : p. 81-86.

96. Yamashita, H., Takenoshita, M., Sakurai, M., Bruick, R.K., Henzel, W.J., Shillinglaw, W., Arnot, D., and Uyeda, K., A glucose-responsive transcription factor that regulates carbohydrate metabolism in the liver. Proceedings of the National Academy of Sciences of the United States of America, 2001. $98(16)$ : p. 9116-21.

97. Ishii, S., IIzuka, K., Miller, B.C., and Uyeda, K., Carbohydrate response element binding protein directly promotes lipogenic enzyme gene transcription. Proceedings of the National Academy of Sciences of the United States of America, 2004. 101(44): p. 15597-15602.

98. Uyeda, K. and Repa, J.J., Carbohydrate response element binding protein, ChREBP, a transcription factor coupling hepatic glucose utilization and lipid synthesis. Cell metabolism, 2006. 4(2): p. 107-110.

99. da Silva Xavier, G., Rutter, G.A., Diraison, F., Andreolas, C., and Leclerc, I., ChREBP binding to fatty acid synthase and L-type pyruvate kinase genes is stimulated by glucose in pancreatic $\beta$-cells. Journal of lipid research, 2006. $47(11)$ : p. 2482-2491.

100. Kawaguchi, T., Osatomi, K., Yamashita, H., Kabashima, T., and Uyeda, K., Mechanism for Fatty Acid "Sparing" Effect on Glucose-induced Transcription REGULATION OF CARBOHYDRATERESPONSIVE ELEMENT-BINDING PROTEIN BY AMP-ACTIVATED PROTEIN KINASE. Journal of Biological Chemistry, 2002. 277(6): p. 3829-3835.

101. Kawaguchi, T., Takenoshita, M., Kabashima, T., and Uyeda, K., Glucose and CAMP regulate the L-type pyruvate kinase gene by phosphorylation/dephosphorylation of the carbohydrate response element binding protein. Proceedings of the National Academy of Sciences, 2001. 98(24): p. 13710-13715. 
102. Iizuka, K., Bruick, R.K., Liang, G., Horton, J.D., and Uyeda, K., Deficiency of carbohydrate response elementbinding protein (ChREBP) reduces lipogenesis as well as glycolysis. Proceedings of the National Academy of Sciences of the United States of America, 2004. 101(19): p. 7281-6.

103. Koo, S.H., Dutcher, A.K., and Towle, H.C., Glucose and insulin function through two distinct transcription factors to stimulate expression of lipogenic enzyme genes in liver. The Journal of biological chemistry, 2001. 276(12): p. $9437-45$.

104. Yamashita, H., Takenoshita, M., Sakurai, M., Bruick, R.K., Henzel, W.J., Shillinglaw, W., Arnot, D., and Uyeda, K., A glucose-responsive transcription factor that regulates carbohydrate metabolism in the liver. Proceedings of the National Academy of Sciences, 2001.98(16): p. 9116-9121.

105. Dentin, R., Benhamed, F., Hainault, I., Fauveau, V., Foufelle, F., Dyck, J.R., Girard, J., and Postic, C., Liver-specific inhibition of ChREBP improves hepatic steatosis and insulin resistance in ob/ob mice. Diabetes, 2006. $55(8)$ : p. 2159-2170.

106. Robert-Cooperman, C.E., Wilson, C.G., and Burkhardt, B.R., PANDER KO mice on high-fat diet are glucose intolerant yet resistant to fasting hyperglycemia and hyperinsulinemia. FEBS Lett, 2011. 585(9): p. 1345-9.

107. Wang, C., Burkhardt, B.R., Guan, Y., and Yang, J., Role of pancreatic-derived factor in type 2 diabetes: evidence from pancreatic beta cells and liver. Nutr Rev, 2012. $70(2)$ : p. $100-6$.

108. Yang, J. and Guan, Y., Family with sequence similarity 3 gene family and nonalcoholic fatty liver disease. J Gastroenterol Hepatol, 2013. 28 Suppl 1: p. 105-11.

109. Cao, X., Yang, C., Lai, F., Hong, Z., Lin, H., Liu, J., and Li, Y., Elevated circulating level of a cytokine, PANcreatic DERived factor, is associated with metabolic syndrome components in a Chinese population. Journal of Diabetes Investigation, 2015.

110. Wilson, C.G., Robert-Cooperman, C.E., and Burkhardt, B.R., PANcreatic-DERived factor: novel hormone PANDERing to glucose regulation. FEBS Lett, 2011. 585(14): p. 2137-43. 
111. Burkhardt, B.R., Yang, M.C., Robert, C.E., Greene, S.R., McFadden, K.K., Yang, J., Wu, J., Gao, Z., and Wolf, B.A., Tissue-specific and glucose-responsive expression of the pancreatic derived factor (PANDER) promoter. Biochim Biophys Acta, 2005. 1730 (3) : p. 215-25.

112. Davies, M.N., O'Callaghan, B.L., and Towle, H.C., Glucose activates ChREBP by increasing its rate of nuclear entry and relieving repression of its transcriptional activity. Journal of Biological Chemistry, 2008. 283(35): p. 2402924038 .

113. Koo, S.H., Dutcher, A.K., and Towle, H.C., Glucose and insulin function through two distinct transcription factors to stimulate expression of lipogenic enzyme genes in liver. J Biol Chem, 2001. 276(12): p. 9437-45.

114. Ma, L., Robinson, L.N., and Towle, H.C., ChREBP•MIx Is the Principal Mediator of Glucose-induced Gene Expression in the Liver. Journal of Biological Chemistry, 2006. 281(39): p. 28721-28730.

115. Ma, L., Tsatsos, N.G., and Towle, H.C., Direct role of ChREBP.MIx in regulating hepatic glucose-responsive genes. J Biol Chem, 2005. 280(12): p. 12019-27.

116. Stoeckman, A.K., Ma, L., and Towle, H.C., MIX is the functional heteromeric partner of the carbohydrate response element-binding protein in glucose regulation of lipogenic enzyme genes. Journal of Biological Chemistry, 2004. $279(15)$ : p. 15662-15669.

117. Tsatsos, N.G. and Towle, H.C., Glucose activation of ChREBP in hepatocytes occurs via a two-step mechanism. Biochemical and biophysical research communications, 2006. 340(2): p. $449-456$.

118. Burkhardt, B.R., Cook, J.R., Young, R.A., and Wolf, B.A., PDX-1 interaction and regulation of the Pancreatic Derived Factor (PANDER, FAMBB) promoter. Biochim Biophys Acta, 2008. $1779(10):$ p. 645-51.

119. Oosterveer, M.H. and Schoonjans, K., Hepatic glucose sensing and integrative pathways in the liver. Cell Mol Life Sci, 2014. 71(8): p. 1453-67. 
120. Harrow, J., Frankish, A., Gonzalez, J.M., Tapanari, E., Diekhans, M., Kokocinski, F., Aken, B.L., Barrell, D., Zadissa, A., and Searle, S., GENCODE: the reference human genome annotation for The ENCODE Project. Genome research, 2012. $22(9)$ : p. 1760-1774.

121. Cheng, C., Alexander, R., Min, R., Leng, J., Yip, K.Y., Rozowsky, J., Yan, K.-K., Dong, X., Djebali, S., and Ruan, Y., Understanding transcriptional regulation by integrative analysis of transcription factor binding data. Genome research, 2012. 22(9): p. 1658-1667.

122. Carninci, P., Sandelin, A., Lenhard, B., Katayama, S., Shimokawa, K., Ponjavic, J., Semple, C.A., Taylor, M.S., Engstrom, P.G., Frith, M.C., Forrest, A.R., Alkema, W.B., Tan, S.L., Plessy, C., Kodzius, R., Ravasi, T., Kasukawa, T., Fukuda, S., Kanamori-Katayama, M., Kitazume, Y., Kawaji, H., Kai, C., Nakamura, M., Konno, H., Nakano, K., Mottagui-Tabar, S., Arner, P., Chesi, A., Gustincich, S., Persichetti, F., Suzuki, H., Grimmond, S.M., Wells, C.A., Orlando, V., Wahlestedt, C., Liu, E.T., Harbers, M., Kawai, J., Bajic, V.B., Hume, D.A., and Hayashizaki, Y., Genomewide analysis of mammalian promoter architecture and evolution. Nat Genet, 2006. 38(6): p. 626-35.

123. Kawaji, H., Frith, M.C., Katayama, S., Sandelin, A., Kai, C., Kawai, J., Carninci, P., and Hayashizaki, Y., Dynamic usage of transcription start sites within core promoters. Genome Biology, 2006. 7 (12).

124. Iizuka, K., Recent progress on the role of ChREBP in glucose and lipid metabolism. Endocr J, 2013. 60(5): p. 543-55.

125. Iizuka, K., Bruick, R.K., Liang, G., Horton, J.D., and Uyeda, K., Deficiency of carbohydrate response elementbinding protein (ChREBP) reduces lipogenesis as well as glycolysis. Proc Natl Acad Sci U S A, 2004. 101(19): p. $7281-6$.

126. Poungvarin, N., Lee, J., Yechoor, V., Li, M., Assavapokee, T., Suksaranjit, P., Thepsongwajja, J., Saha, P., Oka, K., and Chan, L., Carbohydrate response element-binding protein (ChREBP) plays a pivotal role in beta cell glucotoxicity. Diabetologia, 2012. 55(6): p. 1783-1796. 
127. Kursawe, R., Caprio, S., Giannini, C., Narayan, D., Lin, A., D'Adamo, E., Shaw, M., Pierpont, B., Cushman, S.W., and Shulman, G.I., Decreased transcription of ChREBP-alpha/beta isoforms in abdominal subcutaneous adipose tissue of obese adolescents with prediabetes or early type 2 diabetes: associations with insulin resistance and hyperglycemia. Diabetes, 2013. $62(3)$ : p. 837-44.

128. Eissing, L., Scherer, T., Todter, K., Knippschild, U., Greve, J.W., Buurman, W.A., Pinnschmidt, H.O., Rensen, S.S., Wolf, A.M., Bartelt, A., Heeren, J., Buettner, C., and Scheja, L., De novo lipogenesis in human fat and liver is linked to ChREBP-beta and metabolic health. Nat Commun, 2013. 4: p. 1528 .

129. Wilson, C.G., Robert-Cooperman, C.E., and Burkhardt, B.R., PANcreatic-DERived factor: Novel hormone PANDERing to glucose regulation. FEBS Lett, 2011.

130. Zhuang, F., Guan, Q., Gao, L., Yu, C., Tian, L., Wang, J., Fan, Y., Du, D., and Zhao, J., Levels of serum pancreatic derived factor (PANDER) and their correlations with islet beta cell function in newly diagnosed type 2 diabetic patients. Shandong Daxue Xuebao, 2011. 48(5): p. 1-4.

131. Iizuka, K., Miller, B., and Uyeda, K., Deficiency of carbohydrate-activated transcription factor ChREBP prevents obesity and improves plasma glucose control in leptindeficient (ob/ob) mice. Am J Physiol Endocrinol Metab, 2006. $291(2):$ p. E358-64.

132. Metukuri, M.R., Zhang, P., Basantani, M.K., Chin, C., Stamateris, R.E., Alonso, L.C., Takane, K.K., Gramignoli, R., Strom, S.C., O'Doherty, R.M., Stewart, A.F., Vasavada, R.C., Garcia-Ocana, A., and Scott, D.K., ChREBP mediates glucose-stimulated pancreatic beta-cell proliferation. Diabetes, 2012.61(8): p. 2004-15.

133. da Silva Xavier, G., Sun, G., Qian, Q., Rutter, G.A., and Leclerc, I., ChREBP regulates Pdx-1 and other glucosesensitive genes in pancreatic beta-cells. Biochem Biophys Res Commun, 2010. $402(2)$ : p. 252-7. 
134. Rabinovitch, A., Suarez-Pinzon, W., El-Sheikh, A., Sorensen, O., and Power, R.F., Cytokine gene expression in pancreatic islet-infiltrating leukocytes of $B B$ rats: expression of Thl cytokines correlates with beta-cell destructive insulitis and IDDM. Diabetes, 1996. 45(6): p. 749-54.

135. Atkinson, M.A., Bluestone, J.A., Eisenbarth, G.S., Hebrok, M., Herold, K.C., Accili, D., Pietropaolo, M., Arvan, P.R., Von Herrath, M., Markel, D.S., and Rhodes, C.J., How does type 1 diabetes develop?: the notion of homicide or betacell suicide revisited. Diabetes, 2011. 60(5): p. 1370-9.

136. Ratliff, W.A., Athanason, M.G., Chechele, A.C., Kuehl, M.N., Fernandez, A.M., MarElia, C.B., and Burkhardt, B.R., Hepatic nutrient and hormonal regulation of the PANcreaticDERived factor (PANDER) promoter. Molecular and Cellular Endocrinology, 2015.

137. Dutta, P., Genomic Aberrations at the $3 q$ and 14q loci: Investigation of Key Players in Ovarian and Renal Cancer Biology. 2015.

138. Tsai, F.-Y., Keller, G., Kuo, F.C., Weiss, M., Chen, J., Rosenblatt, M., Alt, F.W., and Orkin, S.H., An early haematopoietic defect in mice lacking the transcription factor GATA-2. Nature, 1994. 371(6494): p. 221-226.

139. Lee, Y.-H., Sauer, B., Johnson, P.F., and Gonzalez, F.J., Disruption of the clebp alpha gene in adult mouse liver. Molecular and cellular biology, 1997. 17(10): p. 6014-6022.

140. McLin, V.A., Rankin, S.A., and Zorn, A.M., Repression of Wnt/ $\beta$-catenin signaling in the anterior endoderm is essential for liver and pancreas development. Development, 2007. 134(12): p. 2207-2217.

141. Ikenoue, T., Ijichi, H., Kato, N., Kanai, F., Masaki, T., Rengifo, W., Okamoto, M., Matsumura, M., Kawabe, T., and Shiratori, Y., Analysis of the $\beta$-Catenin/T Cell Factor Signaling Pathway in 36 Gastrointestinal and Liver Cancer Cells. Cancer Science, 2002. 93(11): p. 1213-1220.

142. Turque, N., Plaza, S., Radvanyi, F., Carriere, C., and Saule, S., Pax-QNR/Pax-6, a paired box-and homeoboxcontaining gene expressed in neurons, is also expressed in pancreatic endocrine cells. Molecular endocrinology, 1994. $8(7)$ : p. $929-938$. 
143. Zellmer, S., Schmidt-Heck, W., Godoy, P., Weng, H., Meyer, C., Lehmann, T., Sparna, T., Schormann, W., Hammad, S., and Kreutz, C., Transcription factors ETF, E2F, and SP-1 are involved in cytokine-independent proliferation of murine hepatocytes. Hepatology, 2010. 52(6): p. 2127-2136.

144. Ardito, F., Arena, V., Vellone, M., Grande, G., Pennacchia, I., Majellaro, F., Giovannini, I., Vecchio, F.M., Nuzzo, G., and Giuliante, F., Strong YB-1 Expression Predicts Liver Recurrence Following Resection for Colorectal Metastases. Journal of Gastrointestinal Surgery, 2014. $18(11)$ : p. 1987-1993.

145. Li, X. and Liao, W.S., Cooperative effects of C/EBP-like and NFxB-like binding sites on rat serum amyloid Al gene expression in liver cells. Nucleic acids research, 1992. $20(18)$ : p. 4765-4772.

146. Furlong, E., Rein, T., and Martin, F., YYl and NFI both activate the human p53 promoter by alternatively binding to a composite element, and YYI and EIA cooperate to amplify p53 promoter activity. Molecular and cellular biology, 1996. 16(10): p. 5933-5945.

147. Ozcan, A., Shen, S.S., Hamilton, C., Anjana, K., Coffey, D., Krishnan, B., and Truong, L.D., PAX 8 expression in non-neoplastic tissues, primary tumors, and metastatic tumors: a comprehensive immunohistochemical study. Modern Pathology, 2011. 24(6): p. 751-764.

148. Griffin, M.J. and Sul, H.S., Insulin Regulation of Fatty Acid Synthase Gene Transcription: Roles of USF and SREBP-1C. IUBMB life, 2004. 56(10): p. 595-600.

149. Ishiji, T., Lace, M., Parkkinen, S., Anderson, R., Haugen, T., Cripe, T., Xiao, J., Davidson, I., Chambon, P., and Turek, L., Transcriptional enhancer factor (TEF)-1 and its cell-specific co-activator activate human papillomavirus-16 E6 and $E 7$ oncogene transcription in keratinocytes and cervical carcinoma cells. The EMBO journal, 1992. 11(6): p. 2271 .

150. Terradillos, O., Billet, O., Renard, C.-A., Levy, R., Molina, T., Briand, P., and Buendia, M.A., The hepatitis $B$ virus $X$ gene potentiates c-myc-induced liver oncogenesis in transgenic mice. Oncogene, 1997. 14(4): p. 395-404. 
151. Ng, L., Rüsch, A., Amma, L.L., Nordström, K., Erway, L.C., Vennström, B., and Forrest, D., Suppression of the deafness and thyroid dysfunction in Thrb-null mice by an independent mutation in the Thra thyroid hormone receptor $\alpha$ gene. Human molecular genetics, 2001. 10(23): p. 2701-2708.

152. Berezowsky, C. and Bag, J., Slow troponin C is present in both muscle and nonmuscle cells. Biochemistry and cell Biology, 1992. $70(8)$ : p. 691-697.

153. Altmäe, S., Martinez-Conejero, J., Salumets, A., Simon, C., Horcajadas, J., and Stavreus-Evers, A., Endometrial gene expression analysis at the time of embryo implantation in women with unexplained infertility. Molecular human reproduction, 2010.16(3): p. 178-187.

154. Nerlov, C., The C/EBP family of transcription factors: a paradigm for interaction between gene expression and proliferation control. Trends in cell biology, 2007. 17(7): p. $318-324$.

155. Croniger, C., Leahy, P., Reshef, L., and Hanson, R.W., $C / E B P$ and the control of phosphoenolpyruvate carboxykinase gene transcription in the liver. Journal of Biological Chemistry, 1998. 273(48): p. 31629-31632.

156. Nizielski, S.E., Arizmendi, C., Shteyngarts, A., Farrell, C.J., and Friedman, J.E., Involvement of transcription factor C/EBP-beta in stimulation of PEPCK gene expression during exercise. American Journal of Physiology-Regulatory, Integrative and Comparative Physiology, 1996. 270(5): p. R1005-R1012.

157. O'Brien, R.M., Lucas, P.C., Yamasaki, T., Noisin, E.L., and Granner, D.K., Potential convergence of insulin and cAMP signal transduction systems at the phosphoenolpyruvate carboxykinase (PEPCK) gene promoter through CCAAT/enhancer binding protein (C/EBP). Journal of Biological Chemistry, 1994. $269(48)$ : p. 30419-30428.

158. Mischoulon, D., Rana, B., Bucher, N., and Farmer, S., Growth-dependent inhibition of CCAAT enhancer-binding protein (C/EBP alpha) gene expression during hepatocyte proliferation in the regenerating liver and in culture. Molecular and cellular biology, 1992. 12(6): p. 2553-2560. 
159. Payne, V., Au, W., Lowe, C., Rahman, S., Friedman, J., O'Rahilly, S., and Rochford, J., C/EBP transcription factors regulate SREBPIC gene expression during adipogenesis. Biochem. J, 2010. 425: p. 215-223.

160. Pan, D., The hippo signaling pathway in development and cancer. Developmental cell, 2010. 19(4): p. 491-505.

161. Knight, J., Shepherd, C., Rizzo, S., Brewer, D., Jhavar, S., Dodson, A., Cooper, C., Eeles, R., Falconer, A., and Kovacs, G., TEADI and c-Cbl are novel prostate basal cell markers that correlate with poor clinical outcome in prostate cancer. British journal of cancer, 2008. 99(11): p. $1849-1858$.

162. Maeda, T., Maeda, M., and Stewart, A.F., TEF-1 transcription factors regulate activity of the mouse mammary tumor virus LTR. Biochemical and biophysical research communications, 2002. 296(5): p. 1279-1285.

163. Godsland, I.F., Insulin resistance and hyperinsulinaemia in the development and progression of cancer. Clin Sci (Lond), 2010. $118(5):$ p. 315-32.

164. Calle, E.E. and Thun, M.J., Obesity and cancer. Oncogene, 2004 . 23(38): p. 6365-6378.

165. Schoen, R.E., Tangen, C.M., Kuller, L.H., Burke, G.L., Cushman, M., Tracy, R.P., Dobs, A., and Savage, P.J., Increased blood glucose and insulin, body size, and incident colorectal cancer. Journal of the National Cancer Institute, 1999. 91 (13): p. 1147-1154.

166. Giovannucci, E., Colditz, G.A., Stampfer, M.J., and Willett, W.C., Physical activity, obesity, and risk of colorectal adenoma in women (United States). Cancer Causes and Control, 1996. $7(2)$ : p. 253-263.

167. Coughlin, S.S., Calle, E.E., Teras, L.R., Petrelli, J., and Thun, M.J., Diabetes mellitus as a predictor of cancer mortality in a large cohort of US adults. American journal of epidemiology, 2004. 159(12): p. 1160.

168. Rapp, K., Schroeder, J., Klenk, J., Ulmer, H., Concin, H., Diem, G., Oberaigner, W., and Weiland, S., Fasting blood glucose and cancer risk in a cohort of more than 140,000 adults in Austria. Diabetologia, 2006. 49(5): p. 945-952. 
169. Tran, T.T., Medline, A., and Bruce, W.R., Insulin promotion of colon tumors in rats. Cancer Epidemiology Biomarkers \& Prevention, 1996. 5(12): p. 1013-1015.

170. Corpet, D.E., Jacquinet, C., Peiffer, G., and Taché, S., Insulin injections promote the growth of aberrant crypt foci in the colon of rats. 1997.

171. Steinbach, G., Kumar, S.P., Reddy, B.S., Lipkin, M., and Holt, P.R., Effects of caloric restriction and dietary fat on epithelial cell proliferation in rat colon. Cancer Research, 1993. 53(12): p. 2745.

172. Stolzenberg-Solomon, R.Z., Graubard, B.I., Chari, S., Limburg, P., Taylor, P.R., Virtamo, J., and Albanes, D., Insulin, glucose, insulin resistance, and pancreatic cancer in male smokers. JAMA: the journal of the American Medical Association, 2005. 294(22): p. 2872.

173. Michaud, D.S., Wolpin, B., Giovannucci, E., Liu, S., Cochrane, B., Manson, J.A.E., Pollak, M.N., Ma, J., and Fuchs, C.S., Prediagnostic plasma C-peptide and pancreatic cancer risk in men and women. Cancer Epidemiology Biomarkers \& Prevention, 2007. 16(10): p. 2101-2109.

174. Waldman, T., Kinzler, K.W., and Vogelstein, B., p21 is necessary for the p53-mediated G1 arrest in human cancer cells. Cancer Research, 1995. 55(22): p. 5187.

175. Gartel, A.L., Serfas, M.S., and Tyner, A.L. p21-negative regulator of the cell cycle. 1996. Royal Society of Medicine.

176. Barboza, J.A., Liu, G., Ju, Z., El-Naggar, A.K., and Lozano, G., p21 delays tumor onset by preservation of chromosomal stability. Proceedings of the National Academy of Sciences, 2006. $103(52)$ : p. 19842.

177. Franklin, D.S., Godfrey, V.L., O'Brien, D.A., Deng, C., and Xiong, Y., Functional collaboration between different cyclin-dependent kinase inhibitors suppresses tumor growth with distinct tissue specificity. Molecular and cellular Biology, 2000. 20(16): p. 6147.

178. Philipp, J., Vo, K., Gurley, K.E., Seidel, K., and Kemp, C.J., Tumor suppression by p27Kipl and p2lCipl during chemically induced skin carcinogenesis. Oncogene, 1999. $18(33)$ : p. 4689. 
179. Poole, A.J., Heap, D., Carroll, R.E., and Tyner, A.L., Tumor suppressor functions for the Cdk inhibitor p21 in the mouse colon. Oncogene, 2004. 23(49): p. 8128-8134.

180. Li, L., Luo, Q., Zheng, M., Pan, C., Wu, G., Lu, Y., Feng, B., Chen, X., and Liu, B., P21-activated protein kinase 1 is overexpressed in gastric cancer and induces cancer metastasis. Oncology reports, 2012.

181. Klopfleisch, R. and Gruber, A., Differential expression of cell cycle regulators p21, p27 and p53 in metastasizing canine mammary adenocarcinomas versus normal mammary glands. Research in veterinary science, 2009. 87(1): p. 9196.

182. Klopfleisch, R., von Euler, H., Sarli, G., Pinho, S., Gärtner, F., and Gruber, A., Molecular Carcinogenesis of Canine Mammary Tumors. Veterinary Pathology Online, 2011. $48(1): p .98$.

183. De la Cueva, E., Garcia-Cao, I., Herranz, M., Lopez, P., Garcia-Palencia, P., Flores, J., Serrano, M., FernandezPiqueras, J., and Martin-Caballero, J., Tumorigenic activity of p2lWafl/Cipl in thymic lymphoma. Oncogene, 2006. 25(29): p. 4128-4132.

184. Huang HL, W.B., Yang SB, Shao Y, You WD, Wang WH, Wang MW, Down-regulation of PANDER mRNA in gastric cancer. World Chinese Journal of Digestology, 2008. 16(14): p. 1513-1518.

185. Chandran, U.R., Ma, C., Dhir, R., Bisceglia, M., LyonsWeiler, M., Liang, W., Michalopoulos, G., Becich, M., and Monzon, F.A., Gene expression profiles of prostate cancer reveal involvement of multiple molecular pathways in the metastatic process. BMC Cancer, 2007. 7: p. 64.

186. Shiiba, M., Ishige, S., Saito, Y., Shimizu, T., Minakawa, Y., Kasamatsu, A., Ogawara, K., Uzawa, K., and Tanzawa, H., Down-regulated expression of family with sequence similarity 3, member $B$ (FAM3B), in oral squamous cell carcinoma. Oral Science International, 2012. 9(1): p. 9-16.

187. Li, Z., Mou, H., Wang, T., Xue, J., Deng, B., Qian, L., Zhou, Y., Gong, W., Wang, J.M., and Wu, G., A non-secretory form of FAM3B promotes invasion and metastasis of human colon cancer cells by upregulating Slug expression. Cancer letters, 2013. $328(2):$ p. 278-284. 
188. Kim, H., Park, S., Kang, J.M., Kim, S.J., and Kim, S.-J., Role of FAM3B as a novel tumor suppressor gene in gastric cancer. Cancer Research, 2013. 73(8 Supplement): p. 662662 .

189. Abdul-Ghani, M.A., Lyssenko, V., Tuomi, T., DeFronzo, R.A., and Groop, L., Fasting versus postload plasma glucose concentration and the risk for future type 2 diabetes results from the botnia study. Diabetes Care, 2009. $32(2)$ : p. 281-286.

190. Defronzo, R.A., Ferrannini, E., and Simonson, D.C., Fasting hyperglycemia in non-insulin-dependent diabetes mellitus: contributions of excessive hepatic glucose production and impaired tissue glucose uptake. Metabolism, 1989. 38(4): p. $387-395$. 


\title{
APPENDIX
}

All previously published work contained within this dissertation was included with copyright permissions from the respective publishers. Use of previously published work has been noted within the text. This Appendix contains the terms and conditions of the license agreements for each previously published work.

The following license agreements are included:

\author{
Elsevier (Molecular and Cellular Endocrinology) [136] \\ Elsevier (Structure) [64] \\ BioScientifica Ltd (Journal of Endocrinology) [70]
}


This is a License Agreement between Whitney Ratliff ("You") and Elsevier ("Elsevier") provided by Copyright Clearance Center ("CCC"). The license consists of your order details, the terms and conditions provided by Elsevier, and the payment terms and conditions.

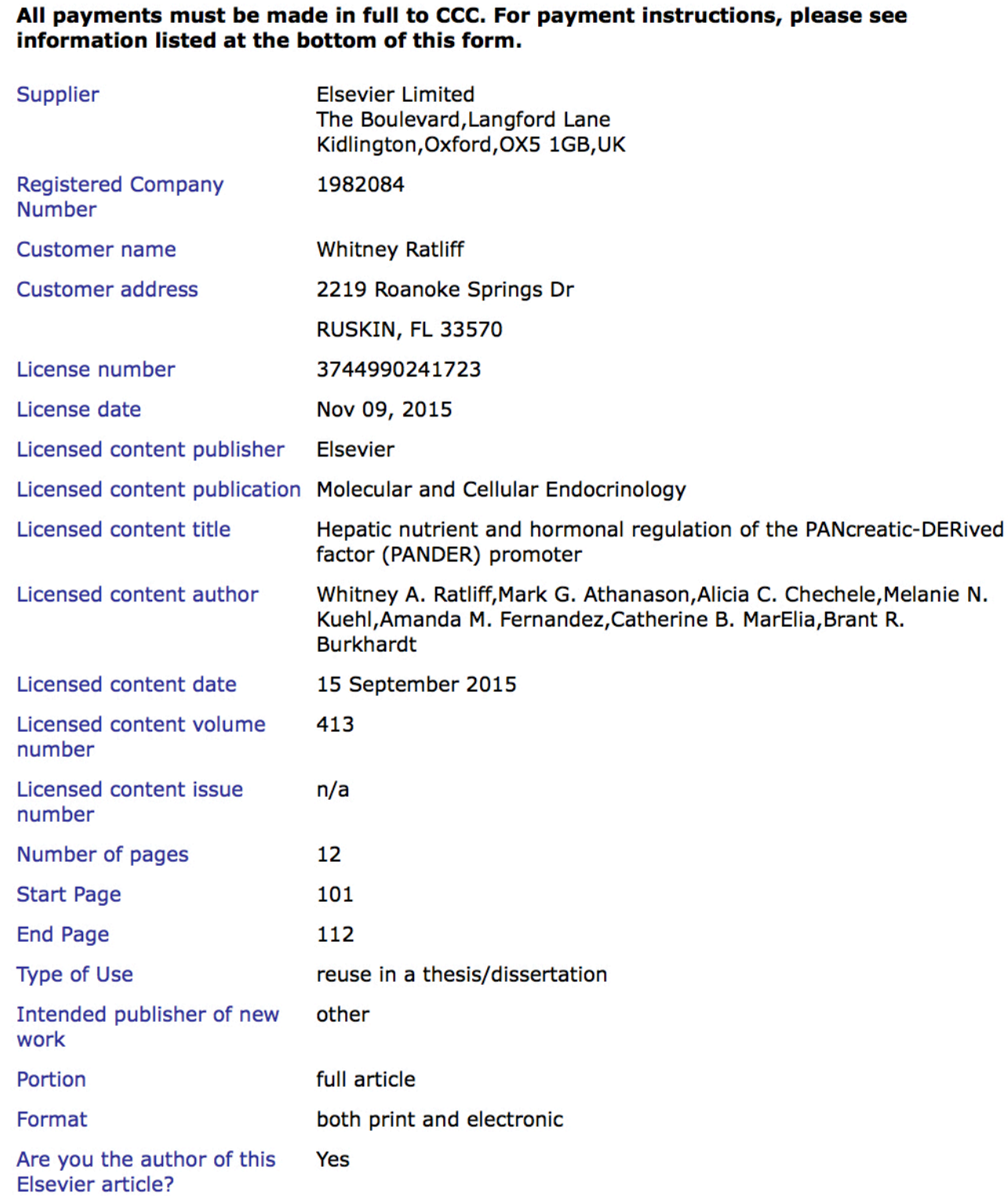

End Page 112

413

$\mathrm{n} / \mathrm{a}$

12

reuse in a thesis/dissertation

other

full article

both print and electronic

Yes 


\begin{tabular}{|c|c|}
\hline Will you be translating? & No \\
\hline $\begin{array}{l}\text { Title of your } \\
\text { thesis/dissertation }\end{array}$ & $\begin{array}{l}\text { Hepatic Nutrient and Hormonal Regulation of the PANcreatic- } \\
\text { DERived Factor (PANDER) Promoter }\end{array}$ \\
\hline Expected completion date & Nov 2015 \\
\hline $\begin{array}{l}\text { Estimated size (number of } \\
\text { pages) }\end{array}$ & 140 \\
\hline Elsevier VAT number & GB 494627212 \\
\hline Permissions price & 0.00 USD \\
\hline VAT/Local Sales Tax & 0.00 USD / 0.00 GBP \\
\hline Total & 0.00 USD \\
\hline
\end{tabular}

\section{INTRODUCTION}

1. The publisher for this copyrighted material is Elsevier. By clicking "accept" in connection with completing this licensing transaction, you agree that the following terms and conditions apply to this transaction (along with the Billing and Payment terms and conditions established by Copyright Clearance Center, Inc. ("CCC"), at the time that you opened your Rightslink account and that are available at any time at http://myaccount.copyright.com).

\section{GENERAL TERMS}

2. Elsevier hereby grants you permission to reproduce the aforementioned material subject to the terms and conditions indicated.

3. Acknowledgement: If any part of the material to be used (for example, figures) has appeared in our publication with credit or acknowledgement to another source, permission must also be sought from that source. If such permission is not obtained then that material may not be included in your publication/copies. Suitable acknowledgement to the source must be made, either as a footnote or in a reference list at the end of your publication, as follows:

"Reprinted from Publication title, Vol /edition number, Author(s), Title of article / title of chapter, Pages No., Copyright (Year), with permission from Elsevier [OR APPLICABLE SOCIETY COPYRIGHT OWNER]." Also Lancet special credit - "Reprinted from The Lancet, Vol. number, Author(s), Title of article, Pages No., Copyright (Year), with permission from Elsevier."

4. Reproduction of this material is confined to the purpose and/or media for which permission is hereby given.

5. Altering/Modifying Material: Not Permitted. However figures and illustrations may be altered/adapted minimally to serve your work. Any other abbreviations, additions, deletions and/or any other alterations shall be made only with prior written authorization of Elsevier Ltd. (Please contact Elsevier at permissions@elsevier.com)

6. If the permission fee for the requested use of our material is waived in this instance, please be advised that your future requests for Elsevier materials may attract a fee. 7. Reservation of Rights: Publisher reserves all rights not specifically granted in the combination of (i) the license details provided by you and accepted in the course of this licensing transaction, (ii) these terms and conditions and (iii) CCC's Billing and Payment terms and conditions.

8. License Contingent Upon Payment: While you may exercise the rights licensed immediately upon issuance of the license at the end of the licensing process for the transaction, provided that you have disclosed complete and accurate details of your proposed use, no license is finally effective unless and until full payment is received from you (either 
by publisher or by CCC) as provided in CCC's Billing and Payment terms and conditions. If full payment is not received on a timely basis, then any license preliminarily granted shall be deemed automatically revoked and shall be void as if never granted. Further, in the event that you breach any of these terms and conditions or any of CCC's Billing and Payment terms and conditions, the license is automatically revoked and shall be void as if never granted. Use of materials as described in a revoked license, as well as any use of the materials beyond the scope of an unrevoked license, may constitute copyright infringement and publisher reserves the right to take any and all action to protect its copyright in the materials.

9. Warranties: Publisher makes no representations or warranties with respect to the licensed material.

10. Indemnity: You hereby indemnify and agree to hold harmless publisher and CCC, and their respective officers, directors, employees and agents, from and against any and all claims arising out of your use of the licensed material other than as specifically authorized pursuant to this license.

11. No Transfer of License: This license is personal to you and may not be sublicensed, assigned, or transferred by you to any other person without publisher's written permission. 12. No Amendment Except in Writing: This license may not be amended except in a writing signed by both parties (or, in the case of publisher, by CCC on publisher's behalf).

13. Objection to Contrary Terms: Publisher hereby objects to any terms contained in any purchase order, acknowledgment, check endorsement or other writing prepared by you, which terms are inconsistent with these terms and conditions or CCC's Billing and Payment terms and conditions. These terms and conditions, together with CCC's Billing and Payment terms and conditions (which are incorporated herein), comprise the entire agreement between you and publisher (and CCC) concerning this licensing transaction. In the event of any conflict between your obligations established by these terms and conditions and those established by CCC's Billing and Payment terms and conditions, these terms and conditions shall control.

14. Revocation: Elsevier or Copyright Clearance Center may deny the permissions described in this License at their sole discretion, for any reason or no reason, with a full refund payable to you. Notice of such denial will be made using the contact information provided by you. Failure to receive such notice will not alter or invalidate the denial. In no event will Elsevier or Copyright Clearance Center be responsible or liable for any costs, expenses or damage incurred by you as a result of a denial of your permission request, other than a refund of the amount(s) paid by you to Elsevier and/or Copyright Clearance Center for denied permissions.

\section{LIMITED LICENSE}

The following terms and conditions apply only to specific license types:

15. Translation: This permission is granted for non-exclusive world English rights only unless your license was granted for translation rights. If you licensed translation rights you may only translate this content into the languages you requested. A professional translator must perform all translations and reproduce the content word for word preserving the integrity of the article.

16. Posting licensed content on any Website: The following terms and conditions apply as follows: Licensing material from an Elsevier journal: All content posted to the web site must maintain the copyright information line on the bottom of each image; A hyper-text must be included to the Homepage of the journal from which you are licensing at http://www.sciencedirect.com/science/journal/xxxxx or the Elsevier homepage for books at http://www.elsevier.com; Central Storage: This license does not include permission for a scanned version of the material to be stored in a central repository such as that provided by 
Heron/XanEdu.

Licensing material from an Elsevier book: A hyper-text link must be included to the Elsevier homepage at http://www.elsevier.com . All content posted to the web site must maintain the copyright information line on the bottom of each image.

Posting licensed content on Electronic reserve: In addition to the above the following clauses are applicable: The web site must be password-protected and made available only to bona fide students registered on a relevant course. This permission is granted for 1 year only. You may obtain a new license for future website posting.

17. For journal authors: the following clauses are applicable in addition to the above:

Preprints:

A preprint is an author's own write-up of research results and analysis, it has not been peerreviewed, nor has it had any other value added to it by a publisher (such as formatting, copyright, technical enhancement etc.).

Authors can share their preprints anywhere at any time. Preprints should not be added to or enhanced in any way in order to appear more like, or to substitute for, the final versions of articles however authors can update their preprints on arXiv or RePEc with their Accepted Author Manuscript (see below).

If accepted for publication, we encourage authors to link from the preprint to their formal publication via its DOI. Millions of researchers have access to the formal publications on ScienceDirect, and so links will help users to find, access, cite and use the best available version. Please note that Cell Press, The Lancet and some society-owned have different preprint policies. Information on these policies is available on the journal homepage. Accepted Author Manuscripts: An accepted author manuscript is the manuscript of an article that has been accepted for publication and which typically includes authorincorporated changes suggested during submission, peer review and editor-author communications.

Authors can share their accepted author manuscript:

- immediately

- via their non-commercial person homepage or blog

- by updating a preprint in arXiv or RePEc with the accepted manuscript

- via their research institute or institutional repository for internal institutional uses or as part of an invitation-only research collaboration work-group

- directly by providing copies to their students or to research collaborators for their personal use

- for private scholarly sharing as part of an invitation-only work group on commercial sites with which Elsevier has an agreement

- after the embargo period

- via non-commercial hosting platforms such as their institutional repository

○ via commercial sites with which Elsevier has an agreement

In all cases accepted manuscripts should:

- link to the formal publication via its DOI

- bear a CC-BY-NC-ND license - this is easy to do

- if aggregated with other manuscripts, for example in a repository or other site, be shared in alignment with our hosting policy not be added to or enhanced in any way to appear more like, or to substitute for, the published journal article. 
Published journal article (JPA): A published journal article (PJA) is the definitive final record of published research that appears or will appear in the journal and embodies all value-adding publishing activities including peer review co-ordination, copy-editing, formatting, (if relevant) pagination and online enrichment.

Policies for sharing publishing journal articles differ for subscription and gold open access articles:

Subscription Articles: If you are an author, please share a link to your article rather than the full-text. Millions of researchers have access to the formal publications on ScienceDirect, and so links will help your users to find, access, cite, and use the best available version. Theses and dissertations which contain embedded PJAs as part of the formal submission can be posted publicly by the awarding institution with DOI links back to the formal publications on ScienceDirect.

If you are affiliated with a library that subscribes to ScienceDirect you have additional private sharing rights for others' research accessed under that agreement. This includes use for classroom teaching and internal training at the institution (including use in course packs and courseware programs), and inclusion of the article for grant funding purposes.

Gold Open Access Articles: May be shared according to the author-selected end-user license and should contain a CrossMark logo, the end user license, and a DOI link to the formal publication on ScienceDirect.

Please refer to Elsevier's posting policy for further information.

18. For book authors the following clauses are applicable in addition to the above: Authors are permitted to place a brief summary of their work online only. You are not allowed to download and post the published electronic version of your chapter, nor may you scan the printed edition to create an electronic version. Posting to a repository: Authors are permitted to post a summary of their chapter only in their institution's repository.

19. Thesis/Dissertation: If your license is for use in a thesis/dissertation your thesis may be submitted to your institution in either print or electronic form. Should your thesis be published commercially, please reapply for permission. These requirements include permission for the Library and Archives of Canada to supply single copies, on demand, of the complete thesis and include permission for Proquest/UMI to supply single copies, on demand, of the complete thesis. Should your thesis be published commercially, please reapply for permission. Theses and dissertations which contain embedded PJAs as part of the formal submission can be posted publicly by the awarding institution with DOI links back to the formal publications on ScienceDirect.

\section{Elsevier Open Access Terms and Conditions}

You can publish open access with Elsevier in hundreds of open access journals or in nearly 2000 established subscription journals that support open access publishing. Permitted third party re-use of these open access articles is defined by the author's choice of Creative Commons user license. See our open access license policy for more information.

Terms \& Conditions applicable to all Open Access articles published with Elsevier: Any reuse of the article must not represent the author as endorsing the adaptation of the article nor should the article be modified in such a way as to damage the author's honour or reputation. If any changes have been made, such changes must be clearly indicated. The author(s) must be appropriately credited and we ask that you include the end user license and a DOI link to the formal publication on ScienceDirect.

If any part of the material to be used (for example, figures) has appeared in our publication with credit or acknowledgement to another source it is the responsibility of the user to ensure their reuse complies with the terms and conditions determined by the rights holder. Additional Terms \& Conditions applicable to each Creative Commons user license: 
CC BY: The CC-BY license allows users to copy, to create extracts, abstracts and new works from the Article, to alter and revise the Article and to make commercial use of the Article (including reuse and/or resale of the Article by commercial entities), provided the user gives appropriate credit (with a link to the formal publication through the relevant DOI), provides a link to the license, indicates if changes were made and the licensor is not represented as endorsing the use made of the work. The full details of the license are available at http://creativecommons.org/licenses/by/4.0.

CC BY NC SA: The CC BY-NC-SA license allows users to copy, to create extracts, abstracts and new works from the Article, to alter and revise the Article, provided this is not done for commercial purposes, and that the user gives appropriate credit (with a link to the formal publication through the relevant DOI), provides a link to the license, indicates if changes were made and the licensor is not represented as endorsing the use made of the work. Further, any new works must be made available on the same conditions. The full details of the license are available at http://creativecommons.org/licenses/by-nc-sa/4.0.

CC BY NC ND: The CC BY-NC-ND license allows users to copy and distribute the Article, provided this is not done for commercial purposes and further does not permit distribution of the Article if it is changed or edited in any way, and provided the user gives appropriate credit (with a link to the formal publication through the relevant DOI), provides a link to the license, and that the licensor is not represented as endorsing the use made of the work. The full details of the license are available at http://creativecommons.org/licenses/by-nc-nd/4.0. Any commercial reuse of Open Access articles published with a CC BY NC SA or CC BY NC ND license requires permission from Elsevier and will be subject to a fee.

Commercial reuse includes:

- Associating advertising with the full text of the Article

- Charging fees for document delivery or access

- Article aggregation

- Systematic distribution via e-mail lists or share buttons

Posting or linking by commercial companies for use by customers of those companies.

\section{Other Conditions:}

\section{v1.8}

Questions? customercare@copyright.com or +1-855-239-3415 (toll free in the US) or +1-978-646-2777. 


\section{ELSEVIER LICENSE} TERMS AND CONDITIONS

This is a License Agreement between Whitney Ratliff ("You") and Elsevier ("Elsevier") provided by Copyright Clearance Center ("CCC"). The license consists of your order details, the terms and conditions provided by Elsevier, and the payment terms and conditions.

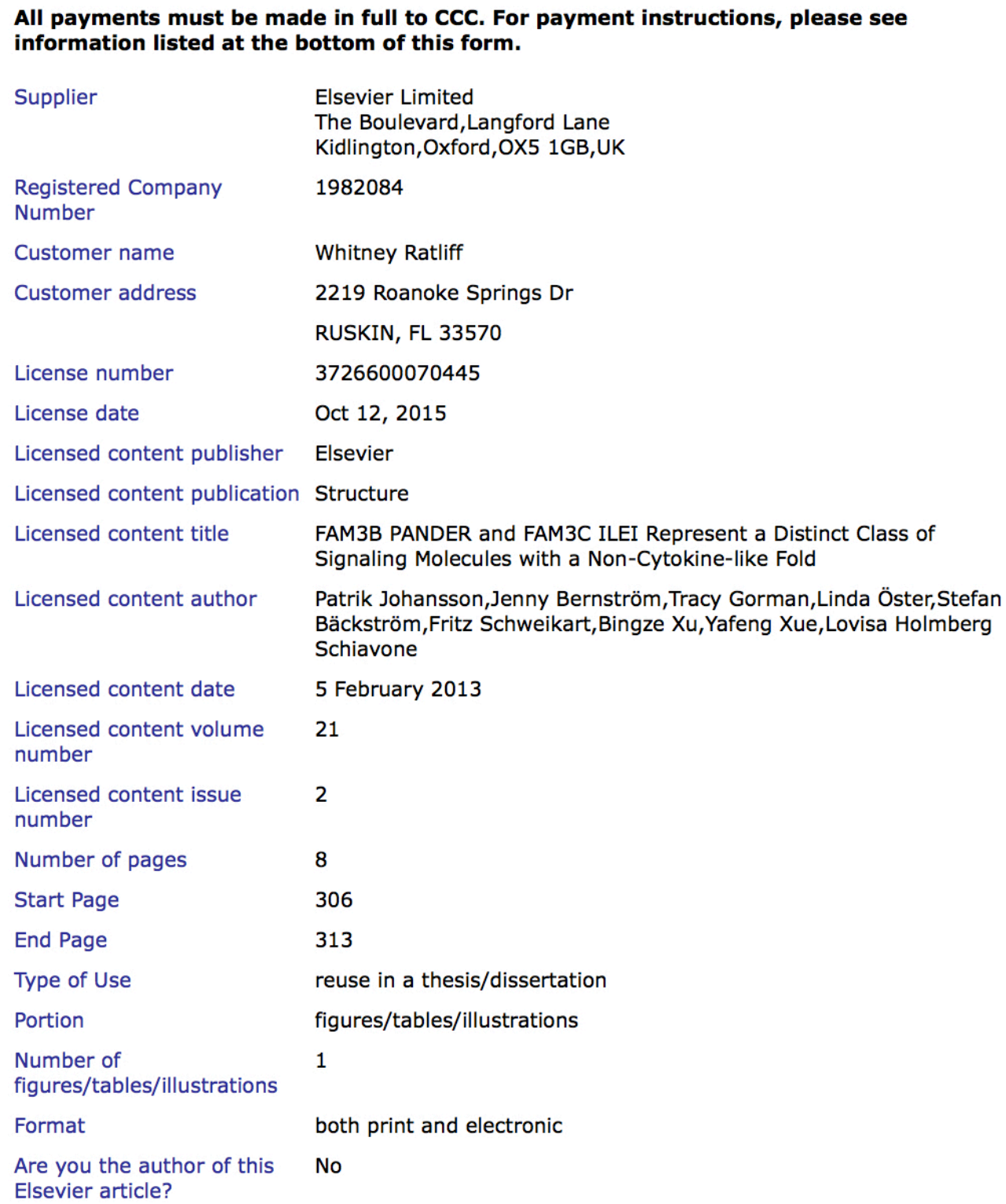

Portion figures/tables/illustrations

1

21

\section{2}

\section{8}

both print and electronic

No 


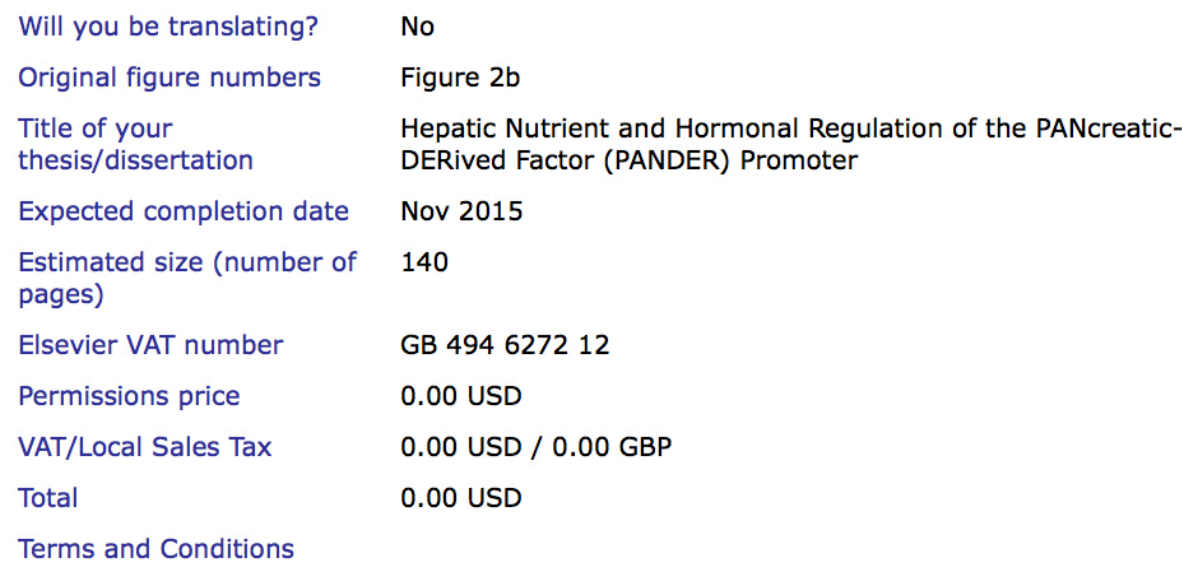

\section{INTRODUCTION}

1. The publisher for this copyrighted material is Elsevier. By clicking "accept" in connection with completing this licensing transaction, you agree that the following terms and conditions apply to this transaction (along with the Billing and Payment terms and conditions established by Copyright Clearance Center, Inc. ("CCC"), at the time that you opened your Rightslink account and that are available at any time at http://myaccount.copyright.com).

\section{GENERAL TERMS}

2. Elsevier hereby grants you permission to reproduce the aforementioned material subject to the terms and conditions indicated.

3. Acknowledgement: If any part of the material to be used (for example, figures) has appeared in our publication with credit or acknowledgement to another source, permission must also be sought from that source. If such permission is not obtained then that material may not be included in your publication/copies. Suitable acknowledgement to the source must be made, either as a footnote or in a reference list at the end of your publication, as follows:

"Reprinted from Publication title, Vol /edition number, Author(s), Title of article / title of chapter, Pages No., Copyright (Year), with permission from Elsevier [OR APPLICABLE SOCIETY COPYRIGHT OWNER]." Also Lancet special credit - "Reprinted from The Lancet, Vol. number, Author(s), Title of article, Pages No., Copyright (Year), with permission from Elsevier."

4. Reproduction of this material is confined to the purpose and/or media for which permission is hereby given.

5. Altering/Modifying Material: Not Permitted. However figures and illustrations may be altered/adapted minimally to serve your work. Any other abbreviations, additions, deletions and/or any other alterations shall be made only with prior written authorization of Elsevier Ltd. (Please contact Elsevier at permissions@elsevier.com)

6. If the permission fee for the requested use of our material is waived in this instance, please be advised that your future requests for Elsevier materials may attract a fee.

7. Reservation of Rights: Publisher reserves all rights not specifically granted in the combination of (i) the license details provided by you and accepted in the course of this licensing transaction, (ii) these terms and conditions and (iii) CCC's Billing and Payment terms and conditions.

8. License Contingent Upon Payment: While you may exercise the rights licensed immediately upon issuance of the license at the end of the licensing process for the 
transaction, provided that you have disclosed complete and accurate details of your proposed use, no license is finally effective unless and until full payment is received from you (either by publisher or by CCC) as provided in CCC's Billing and Payment terms and conditions. If full payment is not received on a timely basis, then any license preliminarily granted shall be deemed automatically revoked and shall be void as if never granted. Further, in the event that you breach any of these terms and conditions or any of CCC's Billing and Payment terms and conditions, the license is automatically revoked and shall be void as if never granted. Use of materials as described in a revoked license, as well as any use of the materials beyond the scope of an unrevoked license, may constitute copyright infringement and publisher reserves the right to take any and all action to protect its copyright in the materials.

9. Warranties: Publisher makes no representations or warranties with respect to the licensed material.

10. Indemnity: You hereby indemnify and agree to hold harmless publisher and CCC, and their respective officers, directors, employees and agents, from and against any and all claims arising out of your use of the licensed material other than as specifically authorized pursuant to this license.

11. No Transfer of License: This license is personal to you and may not be sublicensed, assigned, or transferred by you to any other person without publisher's written permission. 12. No Amendment Except in Writing: This license may not be amended except in a writing signed by both parties (or, in the case of publisher, by CCC on publisher's behalf).

13. Objection to Contrary Terms: Publisher hereby objects to any terms contained in any purchase order, acknowledgment, check endorsement or other writing prepared by you, which terms are inconsistent with these terms and conditions or CCC's Billing and Payment terms and conditions. These terms and conditions, together with CCC's Billing and Payment terms and conditions (which are incorporated herein), comprise the entire agreement between you and publisher (and CCC) concerning this licensing transaction. In the event of any conflict between your obligations established by these terms and conditions and those established by CCC's Billing and Payment terms and conditions, these terms and conditions shall control.

14. Revocation: Elsevier or Copyright Clearance Center may deny the permissions described in this License at their sole discretion, for any reason or no reason, with a full refund payable to you. Notice of such denial will be made using the contact information provided by you. Failure to receive such notice will not alter or invalidate the denial. In no event will Elsevier or Copyright Clearance Center be responsible or liable for any costs, expenses or damage incurred by you as a result of a denial of your permission request, other than a refund of the amount(s) paid by you to Elsevier and/or Copyright Clearance Center for denied permissions.

\section{LIMITED LICENSE}

The following terms and conditions apply only to specific license types: 15. Translation: This permission is granted for non-exclusive world English rights only unless your license was granted for translation rights. If you licensed translation rights you may only translate this content into the languages you requested. A professional translator must perform all translations and reproduce the content word for word preserving the integrity of the article.

16. Posting licensed content on any Website: The following terms and conditions apply as follows: Licensing material from an Elsevier journal: All content posted to the web site must maintain the copyright information line on the bottom of each image; A hyper-text must be included to the Homepage of the journal from which you are licensing at http://www.sciencedirect.com/science/journal/xxxxx or the Elsevier homepage for books at 
http://www.elsevier.com; Central Storage: This license does not include permission for a scanned version of the material to be stored in a central repository such as that provided by Heron/XanEdu.

Licensing material from an Elsevier book: A hyper-text link must be included to the Elsevier homepage at http://www.elsevier.com . All content posted to the web site must maintain the copyright information line on the bottom of each image.

Posting licensed content on Electronic reserve: In addition to the above the following clauses are applicable: The web site must be password-protected and made available only to bona fide students registered on a relevant course. This permission is granted for 1 year only. You may obtain a new license for future website posting.

17. For journal authors: the following clauses are applicable in addition to the above: Preprints:

A preprint is an author's own write-up of research results and analysis, it has not been peerreviewed, nor has it had any other value added to it by a publisher (such as formatting, copyright, technical enhancement etc.).

Authors can share their preprints anywhere at any time. Preprints should not be added to or enhanced in any way in order to appear more like, or to substitute for, the final versions of articles however authors can update their preprints on arXiv or RePEc with their Accepted Author Manuscript (see below).

If accepted for publication, we encourage authors to link from the preprint to their formal publication via its DOI. Millions of researchers have access to the formal publications on ScienceDirect, and so links will help users to find, access, cite and use the best available version. Please note that Cell Press, The Lancet and some society-owned have different preprint policies. Information on these policies is available on the journal homepage.

Accepted Author Manuscripts: An accepted author manuscript is the manuscript of an article that has been accepted for publication and which typically includes authorincorporated changes suggested during submission, peer review and editor-author communications.

Authors can share their accepted author manuscript:

- immediately

- via their non-commercial person homepage or blog

- by updating a preprint in arXiv or RePEc with the accepted manuscript

- via their research institute or institutional repository for internal institutional uses or as part of an invitation-only research collaboration work-group

- directly by providing copies to their students or to research collaborators for their personal use

- for private scholarly sharing as part of an invitation-only work group on commercial sites with which Elsevier has an agreement

- after the embargo period

- via non-commercial hosting platforms such as their institutional repository

- via commercial sites with which Elsevier has an agreement

In all cases accepted manuscripts should:

- link to the formal publication via its DOI

- bear a CC-BY-NC-ND license - this is easy to do

- if aggregated with other manuscripts, for example in a repository or other site, be shared in alignment with our hosting policy not be added to or enhanced in any way to 
appear more like, or to substitute for, the published journal article.

Published journal article (JPA): A published journal article (PJA) is the definitive final record of published research that appears or will appear in the journal and embodies all value-adding publishing activities including peer review co-ordination, copy-editing, formatting, (if relevant) pagination and online enrichment.

Policies for sharing publishing journal articles differ for subscription and gold open access articles:

Subscription Articles: If you are an author, please share a link to your article rather than the full-text. Millions of researchers have access to the formal publications on ScienceDirect, and so links will help your users to find, access, cite, and use the best available version.

Theses and dissertations which contain embedded PJAs as part of the formal submission can be posted publicly by the awarding institution with DOI links back to the formal publications on ScienceDirect.

If you are affiliated with a library that subscribes to ScienceDirect you have additional private sharing rights for others' research accessed under that agreement. This includes use for classroom teaching and internal training at the institution (including use in course packs and courseware programs), and inclusion of the article for grant funding purposes.

Gold Open Access Articles: May be shared according to the author-selected end-user license and should contain a CrossMark logo, the end user license, and a DOI link to the formal publication on ScienceDirect.

Please refer to Elsevier's posting_policy for further information.

18. For book authors the following clauses are applicable in addition to the above: Authors are permitted to place a brief summary of their work online only. You are not allowed to download and post the published electronic version of your chapter, nor may you scan the printed edition to create an electronic version. Posting to a repository: Authors are permitted to post a summary of their chapter only in their institution's repository.

19. Thesis/Dissertation: If your license is for use in a thesis/dissertation your thesis may be submitted to your institution in either print or electronic form. Should your thesis be published commercially, please reapply for permission. These requirements include permission for the Library and Archives of Canada to supply single copies, on demand, of the complete thesis and include permission for Proquest/UMI to supply single copies, on demand, of the complete thesis. Should your thesis be published commercially, please reapply for permission. Theses and dissertations which contain embedded PJAs as part of the formal submission can be posted publicly by the awarding institution with DOI links back to the formal publications on ScienceDirect.

\section{Elsevier Open Access Terms and Conditions}

You can publish open access with Elsevier in hundreds of open access journals or in nearly 2000 established subscription journals that support open access publishing. Permitted third party re-use of these open access articles is defined by the author's choice of Creative Commons user license. See our open access license policy for more information.

Terms \& Conditions applicable to all Open Access articles published with Elsevier: Any reuse of the article must not represent the author as endorsing the adaptation of the article nor should the article be modified in such a way as to damage the author's honour or reputation. If any changes have been made, such changes must be clearly indicated. The author(s) must be appropriately credited and we ask that you include the end user license and a DOI link to the formal publication on ScienceDirect.

If any part of the material to be used (for example, figures) has appeared in our publication with credit or acknowledgement to another source it is the responsibility of the user to ensure their reuse complies with the terms and conditions determined by the rights holder. 
Additional Terms \& Conditions applicable to each Creative Commons user license: CC BY: The CC-BY license allows users to copy, to create extracts, abstracts and new works from the Article, to alter and revise the Article and to make commercial use of the Article (including reuse and/or resale of the Article by commercial entities), provided the user gives appropriate credit (with a link to the formal publication through the relevant DOI), provides a link to the license, indicates if changes were made and the licensor is not represented as endorsing the use made of the work. The full details of the license are available at http://creativecommons.org/licenses/by/4.0.

CC BY NC SA: The CC BY-NC-SA license allows users to copy, to create extracts, abstracts and new works from the Article, to alter and revise the Article, provided this is not done for commercial purposes, and that the user gives appropriate credit (with a link to the formal publication through the relevant DOI), provides a link to the license, indicates if changes were made and the licensor is not represented as endorsing the use made of the work. Further, any new works must be made available on the same conditions. The full details of the license are available at http://creativecommons.org/licenses/by-nc-sa/4.0.

CC BY NC ND: The CC BY-NC-ND license allows users to copy and distribute the Article, provided this is not done for commercial purposes and further does not permit distribution of the Article if it is changed or edited in any way, and provided the user gives appropriate credit (with a link to the formal publication through the relevant DOI), provides a link to the license, and that the licensor is not represented as endorsing the use made of the work. The full details of the license are available at http://creativecommons.org/licenses/by-nc-nd/4.0. Any commercial reuse of Open Access articles published with a CC BY NC SA or CC BY NC ND license requires permission from Elsevier and will be subject to a fee.

Commercial reuse includes:

- Associating advertising with the full text of the Article

- Charging fees for document delivery or access

- Article aggregation

- Systematic distribution via e-mail lists or share buttons

Posting or linking by commercial companies for use by customers of those companies.

\section{Other Conditions:}

v1.8

Questions? customercare@copyright.com or +1-855-239-3415 (toll free in the US) or +1-978-646-2777. 
Copyright permission for Bioscientifica Ltd. (Journal of Endocrinology):

BioScientifica Ltd. LICENSE

TERMS AND CONDITIONS

Oct 15, 2015

This is a License Agreement between Whitney Ratliff ("You") and BioScientifica Ltd. ("BioScientifica Ltd.") provided by Copyright Clearance Center ("CCC"). The license consists of your order details, the terms and conditions provided by BioScientifica Ltd., and the payment terms and conditions.

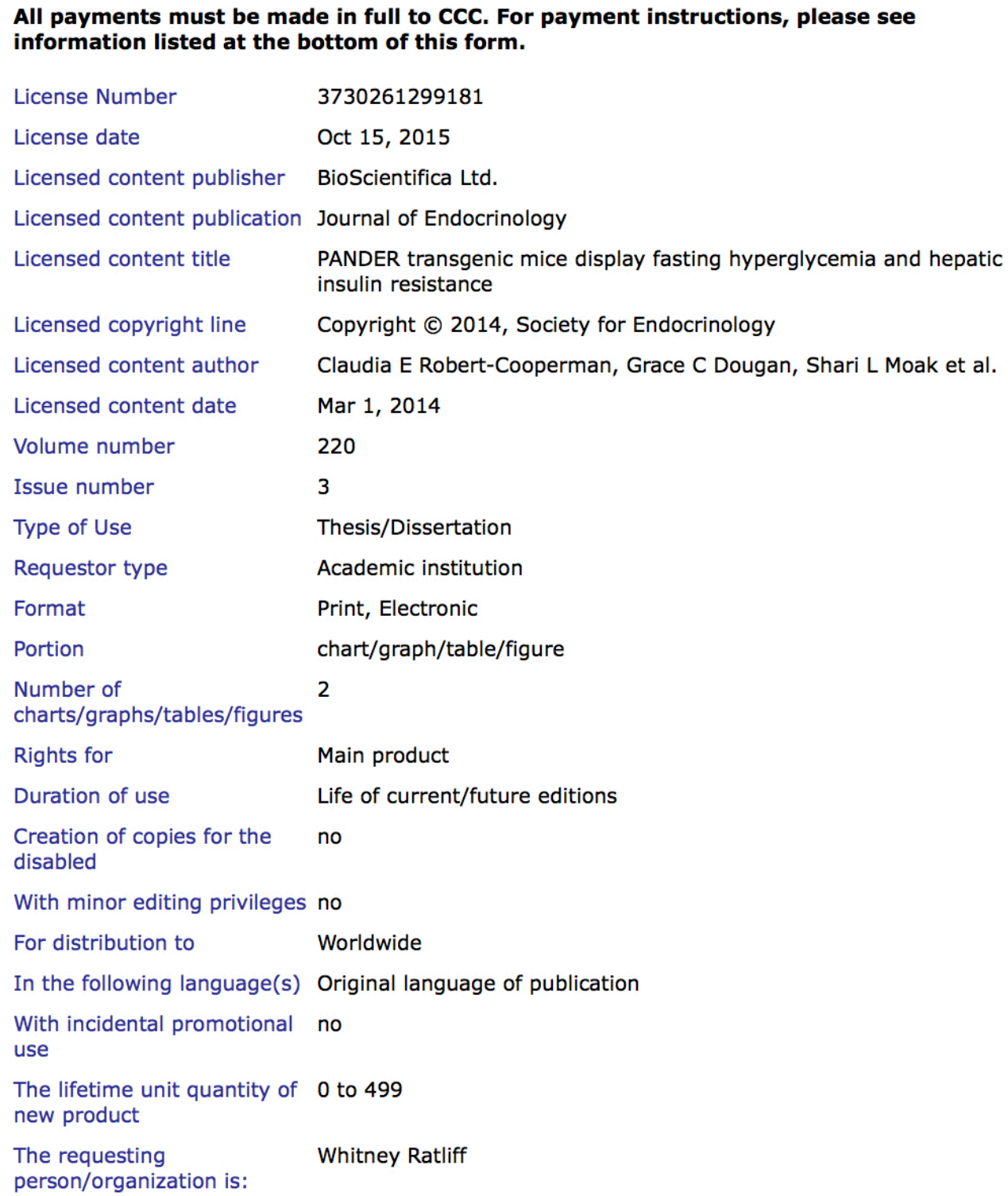




$\begin{array}{ll}\begin{array}{l}\text { Order reference number } \\ \begin{array}{l}\text { Title of your thesis / } \\ \text { dissertation }\end{array}\end{array} & \begin{array}{l}\text { None } \\ \text { Hepatic Nutrient and } \\ \text { DERived Factor (PAN }\end{array} \\ \begin{array}{l}\text { Expected completion date } \\ \text { pages) }\end{array} & \text { Nov } 2015 \\ \text { Billing Type } & 140 \\ \text { Credit card info } & \text { Credit Card } \\ \text { Credit card expiration } & \text { Visa ending in } 6037 \\ \text { Total } & 02 / 2016 \\ \text { Terms and Conditions } & 75.00 \text { USD }\end{array}$

Introduction

The publisher for this copyrighted material is Bioscientifica Ltd. By clicking "accept" in connection with completing this licensing transaction, you agree that the following terms and conditions apply to this transaction (along with the Billing and Payment terms and conditions established by Copyright Clearance Center, Inc. ("CCC"), at the time that you opened your CCC account and that are available at any time at ).

Limited License

The publisher hereby grants to you a non-exclusive license to use this material. Licenses are for one-time use only with a maximum distribution equal to the number that you identified in the licensing process.

Geographic Rights: Scope

Licenses may be exercised anywhere in the world.

Reservation of Rights

The publisher reserves all rights not specifically granted in the combination of (i) the license details provided by you and accepted in the course of this licensing transaction, (ii) these terms and conditions and (iii) CCC's Billing and Payment terms and conditions.

Limited Contingent on Payment

While you may exercise the rights licensed immediately upon issuance of the license at the end of the licensing process for the transaction, provided that you have disclosed complete and accurate details of your proposed use, no license is finally effective unless and until full payment is received from you (either by publisher or by CCC) as provided in CCC's Billing and Payment terms and conditions. If full payment is not received on a timely basis, then any license preliminarily granted shall be deemed automatically revoked and shall be void as if never granted. Further, in the event that you breach any of these terms and conditions or any of CCC's Billing and Payment terms and conditions, the license is automatically revoked and shall be void as if never granted. Use of materials as described in a revoked license, as well as any use of the materials beyond the scope of an unrevoked license, may constitute copyright infringement and publisher reserves the right to take any and all action to protect its copyright in the materials.

Copyright NoticeYou must include the following copyright and permission notice in connection with any reproduction of the licensed material: "Copyright [Original year of publication] [Copyright holder]."

Warranties: None

The publisher makes no representations or warranties with respect to the licensed material and adopts on its own behalf the limitations and disclaimers established by CCC on its behalf in its Billing and Payment terms and conditions for this licensing transaction.

Indemnity 
You hereby indemnify and agree to hold harmless the publisher and CCC, and their respective officers, directors, employees and agents, from and against any and all claims arising out of your use of the licensed material other than as specifically authorized pursuant to this license. In no event shall the publisher or CCC be liable for any special, incidental, indirect or consequential damages of any kind arising out of or in connection with the use of the articles or other material derived from the journals, whether or not advised of the possibility of damage, and on any theory of liability.

No Transfer of License

This license is personal to you and may not be sublicensed, assigned, or transferred by you to any other person without the publisher's written permission.

No Amendment Except in Writing

This license may not be amended except in a writing signed by both parties (or, in the case of the publisher, by CCC on publisher's behalf).

Objection to Contrary Terms

The publisher hereby objects to any terms contained in any purchase order, acknowledgment, check endorsement or other writing prepared by you, which terms are inconsistent with these terms and conditions or CCC's Billing and Payment terms and conditions. These terms and conditions, together with CCC's Billing and Payment terms and conditions (which are incorporated herein), comprise the entire agreement between you and publisher (and CCC) concerning this licensing transaction. In the event of any conflict between your obligations established by these terms and conditions and those established by CCC's Billing and Payment terms and conditions, these terms and conditions shall control. STM Permissions Guidelines

The publisher is a signatory to the STM Guidelines and as such grants permission to other signatory STM publishers to re-use material strictly in accordance with the current STM Guidelines (http://www.stm-assoc.org/permissions-guidelines/).

Other Terms and Conditions:

V.1 10.26/12

Questions? customercare@copyright.com or +1-855-239-3415 (toll free in the US) or +1-978-646-2777. 